\title{
CONDITION MONITORING OF AN AXIAL PISTON PUMP UTILIZING THE KALMAN FILTER
}

A Dissertation presented to the Faculty of the Mechanical

and Aerospace Engineering Department

at the University of Missouri

In Partial Fulfillment

of the Requirements for the Degree

Doctor of Philosophy

by

TYLER A. SHINN

Dr. Roger Fales, Dissertation Supervisor

MAY 2018 
The undersigned, appointed by the dean of the Graduate School, have examined the dissertation entitled

\section{CONDITION MONITORING OF AN AXIAL PISTON PUMP UTILIZING THE KALMAN FILTER}

presented by Tyler Shinn,

a candidate for the degree of Doctor of Philosophy

and hereby certify that, in their opinion, it is worthy of acceptance.

Professor Roger Fales

Professor Craig Kluever

Professor Noah Manring

Professor Ming Xin

Professor Stephen Montgomery-Smith 


\section{ACKNOWLEDGEMENTS}

I would like to thank Dr. Roger Fales for his continued guidance and support throughout my graduate school career through several projects. It has been my privilege to be mentored by him.

I would like to thank the industry funding that was provided to this project and provided equipment and mentorship throughout my time working on this research. The funding has provided me an opportunity to gain invaluable experience and present my work at two conferences.

I would like to thank the undergraduate student research assistants Jacob Rusteberg, Levi Manring, Brandon Casteel, Jacob Ash, and Stuart Belcke for their hard work on helping me manage the experimental setup and data acquisition system.

Finally, I would like to thank my parents, Kent and Cindy Shinn, who have encouraged me from a young age to pursue my dreams. They have consistently shown me support in any endeavor I have undertaken. I would not be where I was today without their guidance. 


\section{TABLE OF CONTENTS}

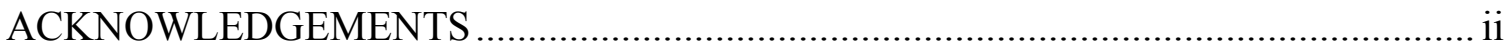

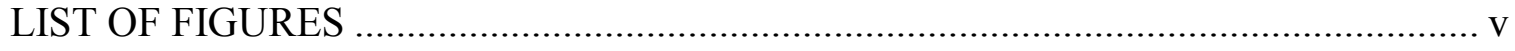

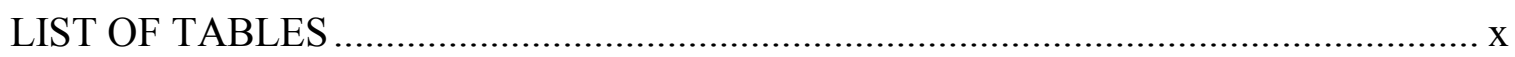

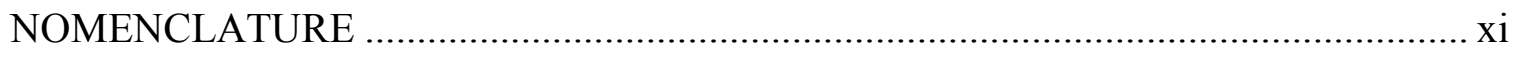

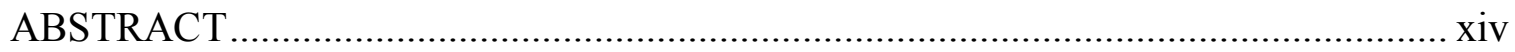

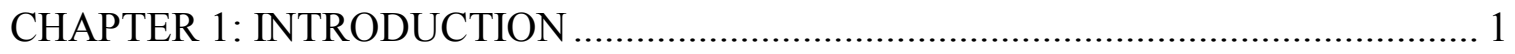

1.1. Axial Piston Pumps ……………………….............................................. 1

1.2. Approaches to Condition Monitoring of Hydraulic Systems ................................ 2

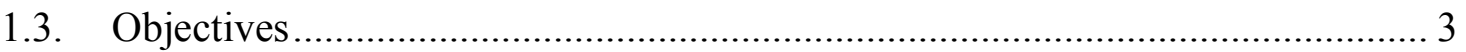

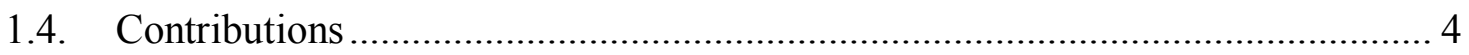

1.5. Overview of the Paper ............................................................................... 5

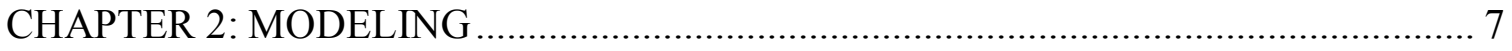

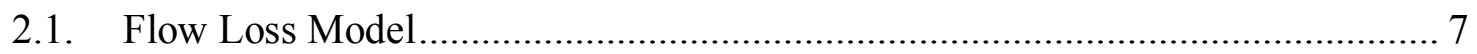

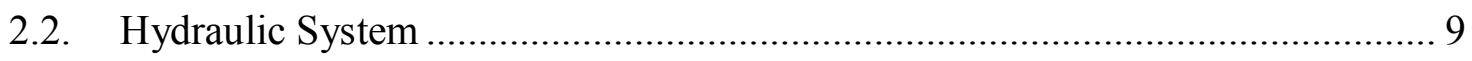

2.2.1. First Order Model ................................................................................ 13

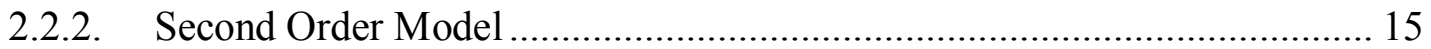

2.2.3. Eighth Order Model .............................................................................. 20

2.3. Augmenting Model to Include Parameter Estimation.......................................... 22

2.4. Discharge Flow Model Validation ............................................................... 23

CHAPTER 3: FLOW LOSS ESTIMATION TECHNIQUES........................................... 27

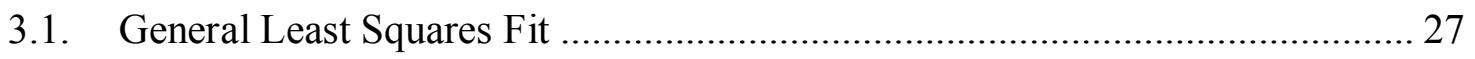

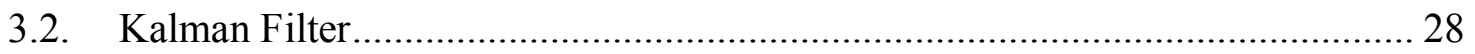

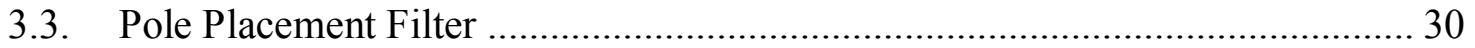

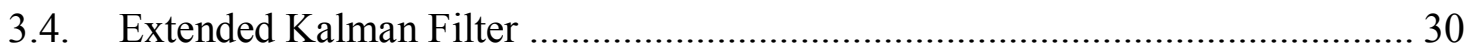

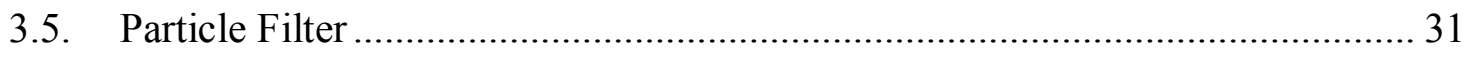

3.6. Unscented Kalman Filter....................................................................... 34

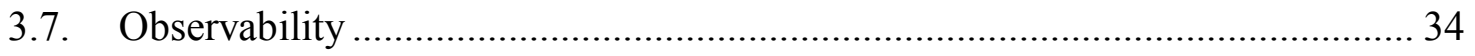


CHAPTER 4: SIMULATION RESULTS AND DISCUSSION .................................. 39

4.1. Eighth Order Model State Estimation .......................................................... 39

4.2. Eighth Order Model Parameter Estimation ................................................... 44

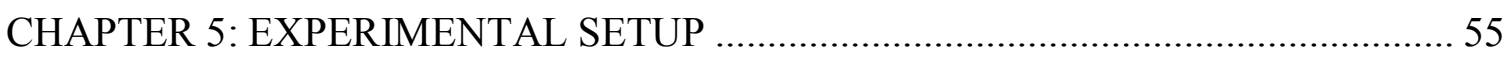

CHAPTER 6: EXPERIMENTAL RESULTS AND DISCUSSION ............................. 57

6.1. First Order Model Results ...................................................................... 57

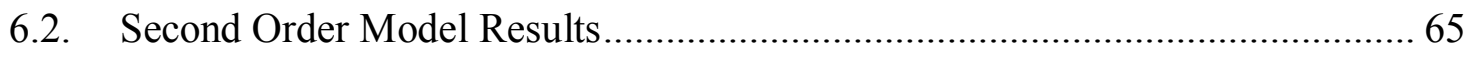

6.3. Flow Loss Estimation without Swash Plate Angle Measurement.................... 75

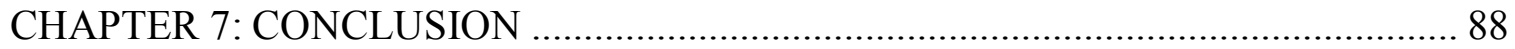

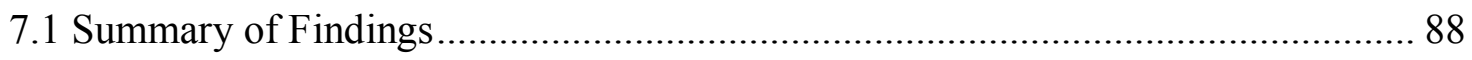

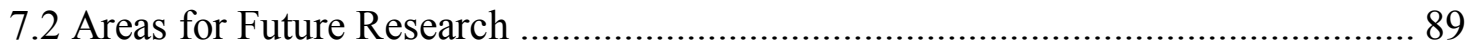

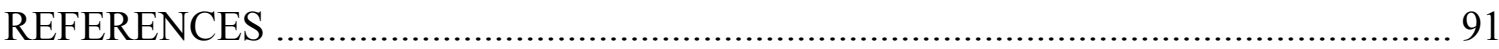

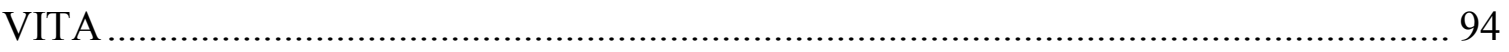




\section{LIST OF FIGURES}

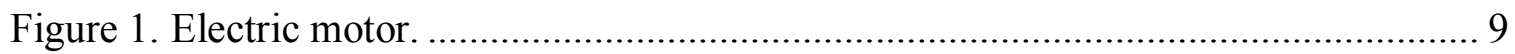

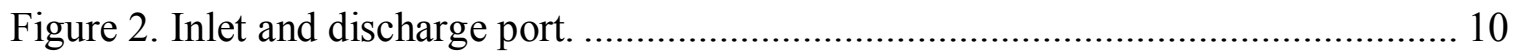

Figure 3. Discharge flow meter and steering system. ............................................ 10

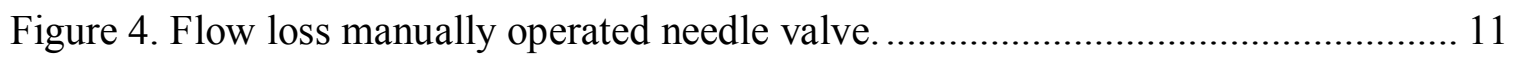

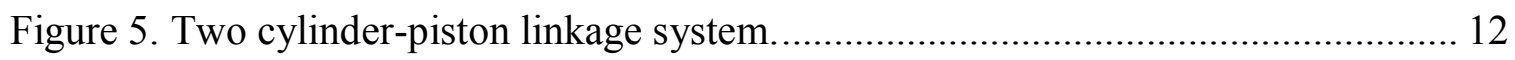

Figure 6. Hydraulic circuit for pump discharge pressure model. ................................. 13

Figure 7. Hydraulic circuit for discharge pressure and swash plate model. .................... 16

Figure 8. Hydraulic circuit for high fidelity model............................................... 21

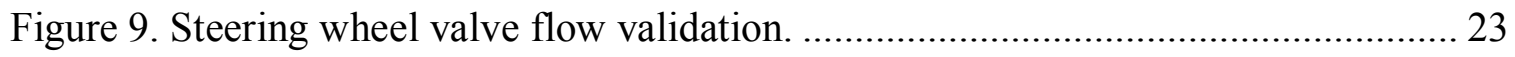

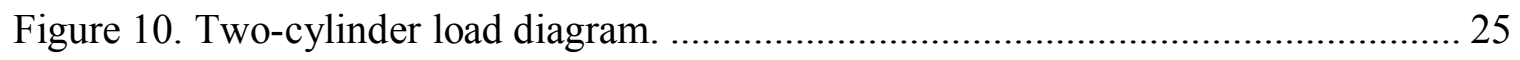

Figure 11. Comparison of Kalman and Particle filter for estimating discharge pressure. 40

Figure 12. Comparison of Kalman and Particle filter for estimating control pressure.... 40

Figure 13. Comparison of Kalman and Particle filter for estimating load pressure. ....... 41

Figure 14. Comparison of Kalman and Particle filter for estimating valve position........ 41

Figure 15. Comparison of Kalman and Particle filter for estimating valve velocity....... 42

Figure 16. Comparison of Kalman and Particle filter for estimating actuator position.... 42

Figure 17. Comparison of Kalman and Particle filter for estimating actuator velocity.... 43

Figure 18. Comparison of Kalman and Particle filter for estimating motor load velocity.

Figure 19. Discharge Pressure estimation for simulation data. .................................. 46

Figure 20. Control Pressure estimation for simulation data. ..................................... 46

Figure 21. Load Pressure estimation for simulation data. ........................................ 47 
Figure 22. Control actuator valve position estimation for simulation data..................... 47

Figure 23. Control actuator valve velocity estimation for simulation data..................... 48

Figure 24. Piston position estimation for simulation data. .......................................... 48

Figure 25. Piston velocity estimation for simulation data. ....................................... 49

Figure 26. Output motor velocity estimation for simulation data.............................. 49

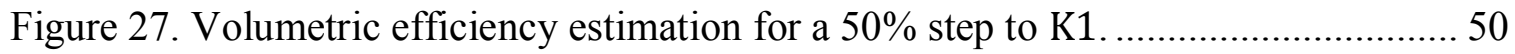

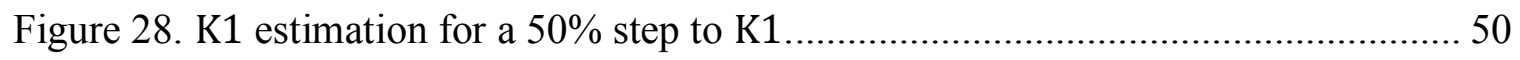

Figure 29. Volumetric efficiency estimation for a 50\% step to $\mathrm{K} 1$ and $\mathrm{K} 2 \ldots \ldots \ldots \ldots \ldots \ldots . . . . . . . . . .51$

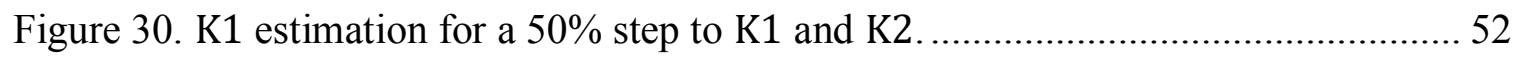

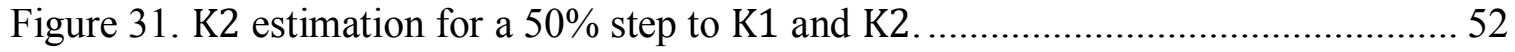

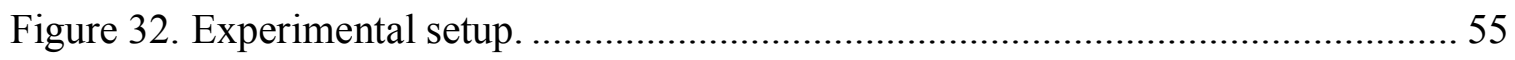

Figure 33. (a) Discharge pressure and load pressure, (b) steering position, (c) flow loss valve flow, and (d) swash plate angle measurements from experimental testing............ 57

Figure 34. PPF (a) state and (b) parameter estimation............................................ 58

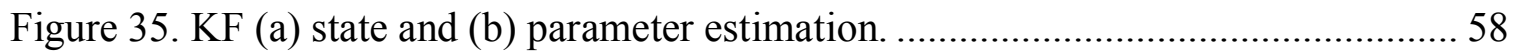

Figure 36. EKF (a) state and (b) parameter estimation............................................ 59

Figure 37. UKF (a) state and (b) parameter estimation. .............................................5 59

Figure 38. Comparison of all four filters for (a) state and (b) parameter estimation........ 60

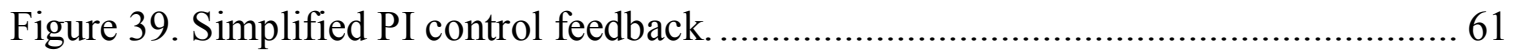

Figure 40. Experimental test for (a) discharge pressure (b) steering velocity (c) swash plate position (d) cylinder displacement (e) discharge flow Rate (f) leakage valve Flow Rate. 62 Figure 41. Discharge pressure estimation from single state model utilizing the EKF. .... 64 Figure 42. Flow loss coefficient estimation from single state model utilizing the EKF. . 64 
Figure 43. Flow loss estimation from single state model utilizing the EKF. 65

Figure 44. Discharge pressure estimation from the two state model utilizing the EKF... 66

Figure 45. Swash plate angle estimation from the two state model utilizing the EKF.... 67

Figure 46. Flow loss coefficient estimation from the two state model utilizing the EKF.67

Figure 47. Flow loss estimation from the two state model utilizing the EKF. 68

Figure 48. Discharge pressure estimation from the two state model utilizing the UKF... 68

Figure 49. Swash plate angle estimation from the two state model utilizing the UKF. ... 69

Figure 50. Comparison of EKF and UKF estimation of flow loss coefficient utilizing the two state model.

Figure 51. Discharge pressure estimation from the two state model utilizing the EKF with scaled flow loss model. 70

Figure 52. Swash plate angle estimation from the two state model utilizing the EKF with scaled flow loss model. 71

Figure 53. Flow loss coefficient estimation from the two state model utilizing the EKF with scaled flow loss model. 71

Figure 54. Flow loss estimation from the two state model utilizing the EKF with scaled flow loss model. 72

Figure 55. Discharge pressure estimation from the two state model utilizing the EKF with offset flow loss model. 72

Figure 56. Swash plate estimation from the two state model utilizing the EKF with offset flow loss model. 73

Figure 57. Flow loss coefficient estimation from the two state model utilizing the EKF with offset flow loss model. 73 
Figure 58. Flow loss estimation from the two state model utilizing the EKF with offset flow loss model. 74

Figure 59. Comparison of $\mathrm{K} 2$ estimation from the first order model to the second order model... 75

Figure 60. Additional Component for measuring control pressure. 76

Figure 61. (a) Control pressure measurement during turning and (b) FFT of measurement from 38 seconds to 40 seconds. 77

Figure 62. Comparison of filtered control pressure to unfiltered. 78

Figure 63. Discharge pressure estimation from the two state model utilizing the EKF with control pressure measured. 79

Figure 64. Swash plate angle estimation from the two state model utilizing the EKF with control pressure measured. 79

Figure 65. Flow loss coefficient estimation from the two state model utilizing the EKF with control pressure measured. 80

Figure 66. Flow loss estimation from the two state model utilizing the EKF with control pressure measured. 80

Figure 67. Comparison of flow loss estimation for four different estimations. 81

Figure 68. Experimental test for (a) discharge pressure (b) steering velocity (c) swash plate angle (d) cylinder displacement (e) discharge flow rate (f) leakage valve flow rate........ 84 Figure 69. Comparison of measured flow to estimated flow across the steering wheel valve.

Figure 70. Discharge pressure estimation when swash plate angle is at maximum displacement (shaded portion indicates the maximum swashplate angle condition). ...... 86 
Figure 71. Flow loss coefficient estimation when swash plate angle is at maximum displacement (shaded portion indicates the maximum swashplate angle condition). ...... 86 


\section{LIST OF TABLES}

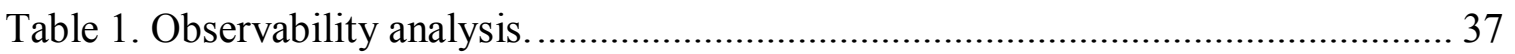

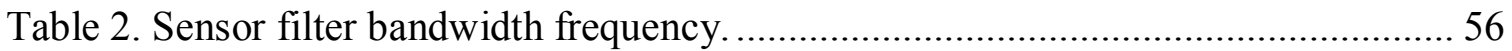

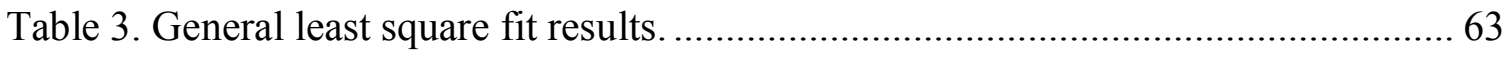

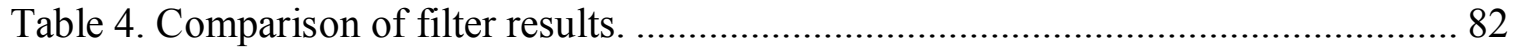




\section{NOMENCLATURE}

\begin{tabular}{|c|c|}
\hline$A_{b p}$ & : Bias piston cross sectional area \\
\hline$A_{c p}$ & : Control piston cross sectional area \\
\hline$A_{\text {cso }}$ & : Control spool valve cross sectional area \\
\hline $\boldsymbol{A}_{\boldsymbol{k}}$ & : Linearized, discrete state transition matrix \\
\hline$A_{p}$ & : Piston cross sectional area \\
\hline$A_{v}$ & : Valve area cross sectional area for orifice equation \\
\hline$b_{y}$ & : Spool valve viscous friction coefficient \\
\hline $\boldsymbol{B}_{\boldsymbol{k}}$ & : Linearized, discrete input matrix \\
\hline$C_{d}$ & : Discharge coefficient \\
\hline$C_{i}$ & : Swivel torque swash plate angle coefficient \\
\hline$C_{\boldsymbol{k}}$ & : Linearized, discrete output state coefficient matrix \\
\hline$C_{p}$ & : Swivel torque discharge pressure coefficient \\
\hline$f$ & : Nonlinear discrete state dynamics vector \\
\hline$g$ & : Nonlinear discrete output vector \\
\hline $\boldsymbol{I}$ & Identity matrix \\
\hline$J$ & : Swash plate angle angular momentum of inertia \\
\hline$J_{w}$ & : Cylinder system wedge angular momentum of inertia \\
\hline$k_{b p}$ & : Bias piston spring rate \\
\hline$k_{c s}$ & : Control spool valve spring rate \\
\hline$K_{1}$ & : Low Reynolds number flow coefficient \\
\hline$K_{2}$ & : High Reynolds number flow coefficient \\
\hline$K_{3}$ & : Scaling flow loss coefficient \\
\hline$K_{4}$ & : Offset flow loss coefficient \\
\hline$K_{c}$ & : Control volume flow loss coefficient \\
\hline $\boldsymbol{K}_{\boldsymbol{k}}$ & : Kalman gain matrix \\
\hline$K_{q}$ & : Control volumetric flow coefficient \\
\hline$K_{w}$ & : Steering wheel turning speed to discharge flow slope coefficient \\
\hline$L_{1}$ & : Constant length in cylinder apparatus \\
\hline
\end{tabular}




\begin{tabular}{|c|c|}
\hline$L_{2}$ & : Constant length in cylinder apparatus \\
\hline$L_{3}$ & : Constant length in cylinder apparatus \\
\hline$L_{4}$ & : Constant length in cylinder apparatus \\
\hline$L_{2 a}$ & : Length from center of swash plate rotation to actuator distance \\
\hline$L_{L}$ & : Left cylinder length \\
\hline$L_{R}$ & : Right cylinder length \\
\hline$m$ & : Control spool valve mass \\
\hline$m_{p s}$ & : Piston and slipper mass \\
\hline$n_{k}$ & : Measurement noise \\
\hline$N_{e f f}$ & : Effective sample size \\
\hline$N_{p}$ & : Number of pistons \\
\hline$N_{S}$ & : Number of replacements \\
\hline $\boldsymbol{O}$ & : Observability matrix \\
\hline$P$ & : Pressure \\
\hline $\boldsymbol{P}_{\boldsymbol{k}}$ & : State covariance error matrix \\
\hline$P_{A}$ & : Chamber A pressure \\
\hline$P_{B}$ & : Chamber B pressure \\
\hline$P_{C}$ & : Control pressure \\
\hline$P_{D}$ & : Discharge pressure \\
\hline$P_{L}$ & : Load pressure \\
\hline$P_{M}$ & : Margin pressure \\
\hline$Q$ & : Volumetric flow \\
\hline$Q_{C}$ & : Control volumetric flow \\
\hline$Q_{D}$ & : Discharge volumetric flow \\
\hline$Q_{D, e s t}$ & : Estimated discharge volumetric flow \\
\hline$Q_{\text {High }}$ & : High Reynolds number volumetric flow \\
\hline$Q_{k}$ & : Process noise covariance matrix \\
\hline$Q_{L}$ & : Discharge volumetric flow loss \\
\hline$Q_{L, C}$ & : Control volumetric flow loss \\
\hline
\end{tabular}




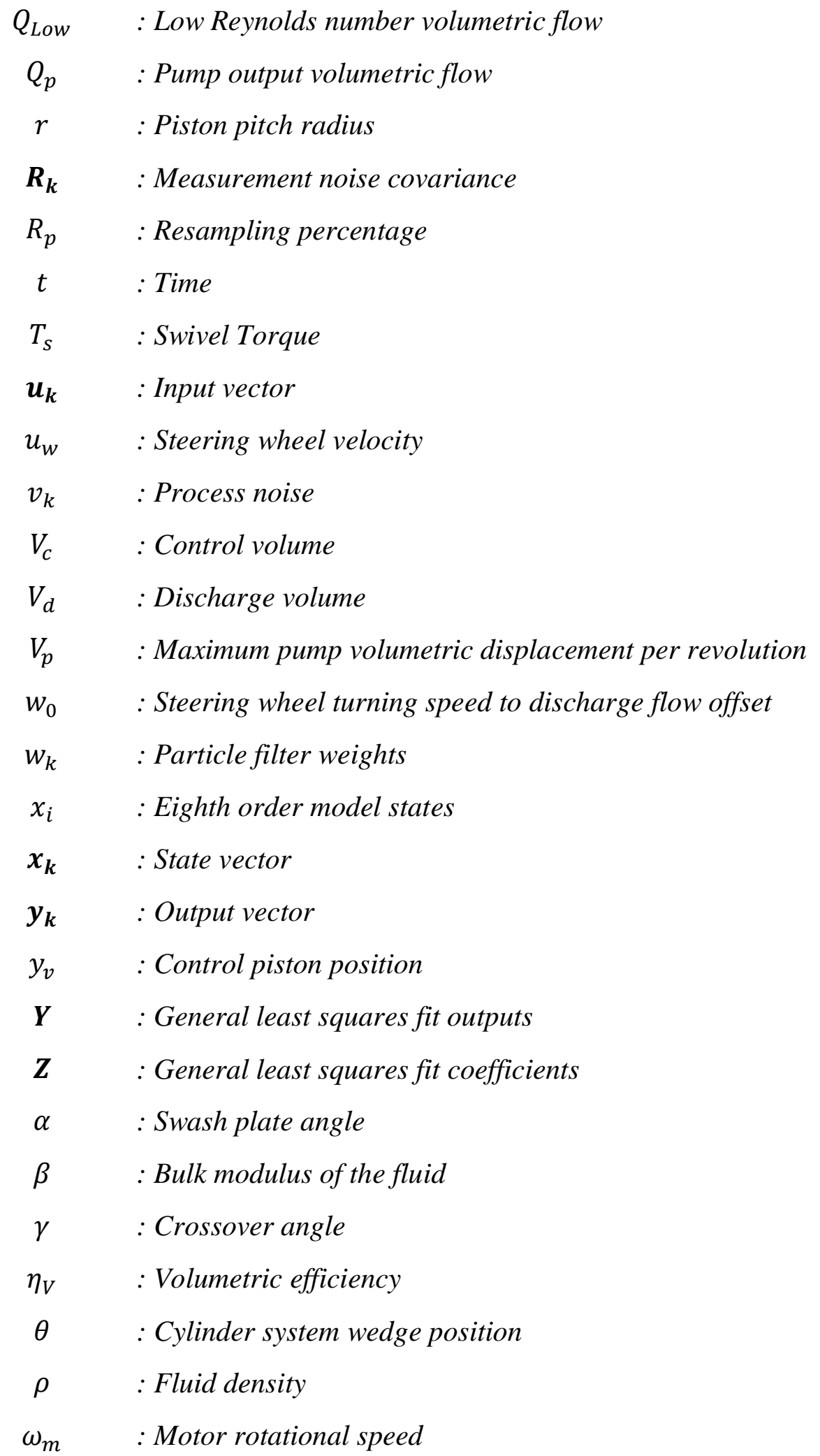




\title{
CONDITION MONITORING OF AN AXIAL PISTON PUMP UTILIZING THE KALMAN FILTER
}

\section{Tyler Shinn}

\section{Dr. Roger Fales, Dissertation Supervisor}

\begin{abstract}
Condition monitoring of a hydraulic pump is an essential process for maximum operational time and pump life longevity. One method of condition monitoring is to estimate parameters characterized by flow losses. Even in a lab environment, direct flow loss measurement may be impractical. Therefore, a method of estimation must be utilized. Filters, such as the Kalman Filter have been implemented in numerous engineering applications to estimate states and parameters. A first order, nonlinear model of the pump discharge pressure, along with several measurements from experimental tests, have been utilized to implement and compare five filters: a pole placement filter (PPF), Kalman Filter (KF), Extended Kalman Filter (EKF), particle filter (PF), and Unscented Kalman Filter (UKF). The discharge pressure, swash plate angle, and a flow loss parameter within the model are estimated using these filters. An eighth order model has also been utilized to test estimation of multiple flow loss parameters (low and high Reynolds number flow losses) simultaneously. The KF, EKF, and PF is utilized for this model, using simulation data. A least squares fit, utilizing the volumetric efficiency of the pump and considering low and high Reynolds number flow losses, has also been calculated and compared to the filter results for the experimental data. Results show that flow losses can be tracked utilizing a simple first or second order model utilizing standard filtering techniques such as the EKF.




\section{CHAPTER 1: INTRODUCTION}

\subsection{Axial Piston Pumps}

The axial piston pump has a wide range of applications in mining, off highway, and construction machinery. The pumps are also extensively used in the aerospace sector. The pump is widely used because it is a load sensing pump. Load sensing pumps feed the load pressure back to the pump to automatically adjust the pump discharge pressure to maintain a specific pressure drop, and therefore flow, across an orifice. This feedback control system is done solely through an hydromechanical system - no sensors or electronics are necessary for the pump to maintain the pressure drop which is extremely advantageous. Axial piston pumps are also extremely efficient, capable of reaching efficiencies over $90 \%$.

High uptime of these pumps is crucial to be cost effective. Downtime for repair and part replacement can reach tens of thousands of dollars per hour due to the high cost environment the pumps are often utilized for. Repairs are not only costly but time consuming also. Catastrophic failure of a pump can damage the entire hydraulic system, requiring the whole system to be replaced. Predicting pump degradation and catastrophic failure has the potential to be a huge cost reduction. Measurement of the pump state through the use of sensors can be used to determine the level of pump degradation. However, the pump housing is typically not designed to allow sensor placements to measure the numerous components moving within an axial piston pump. Flow meters are also not appropriate for many applications. The only information that can normally be measured outside of the lab is the motor/engine speed driving the pump and the pump discharge 
pressure. These sensor limitations make it difficult to discern the state of the pump during operation.

\subsection{Approaches to Condition Monitoring of Hydraulic Systems}

There are many approaches to monitoring hydraulic systems. Wavelet analysis has been utilized for fault diagnosis in pumps [1-5]. While this method has been shown to be effective, the major drawback is sampling time. High sample rates are required to utilize wavelet analysis, which may be feasible in a laboratory setting but often impractical due to cost limitations in industry application. Note that as microprocessor and sensor technology improve, wavelet analysis for condition monitoring could become more viable.

Stochastic estimation methods like the Kalman filter (KF) have been widely applied since the 1960s to monitor systems. The KF, and extended Kalman filter (EKF), has been utilized for hydraulic systems [6-11] and other systems as well [12-15]. Both the KF and EKF have been demonstrated to be appropriate for a wide range of applications, but they have drawbacks. The KF and EKF have reduced performance in large variable systems. The ensemble Kalman filter has been shown to handle large variable systems better [1619]. The KF and EKF estimation is based on linear models. Both the particle filter (PF) [20] and unscented Kalman filter (UKF) [21] are filtering algorithms utilizing only the nonlinear model.

Two previous conference papers have been submitted over this work. A conference paper was submitted to the 2015 ASME/BATH Symposium on Fluid Power and Motion Control that demonstrated estimating simulated states of an eighth order pump model, given only pressure measurements [22]. The KF was compared to the PF. The two estimation techniques were found to have comparable, adequate estimation. The PF did 
improve on some estimation, namely when a state saturated. However, the PF runtime was much more significant than the KF, taking at least 2 times longer than the KF and upwards of 156 times as long as the KF depending on the tuning parameters selected. Another conference paper was submitted to the 2016 ASME/BATH Symposium on Fluid Power and Motion control that compared simulated state and parameter estimation of an eighth order model utilizing the KF and EKF [23]. For single parameter estimation, the KF and EKF compared well and had adequate estimation. However, for two parameter estimation the KF diverged. The EKF showed promise for estimating all states and two parameters simultaneously.

\subsection{Objectives}

1. Develop accurate model with realistic computational run and solver step time. Pump components can have very fast dynamics. Valves within the pump can oscillate at $0.5 \mathrm{kHz}$ or more. Pressure rise rate equations can change extremely rapidly. These fast dynamics necessarily require small step times for accurate model simulation, which leads to longer run times. One goal of this work is to be able to estimate flow losses in real time. This goal will require a model that runs at larger step times and is still be stable.

2. Determine necessary sensors for flow loss estimation. There are many components and pressure dynamics within an axial piston pump. However, the pump housing presents a barrier to placing sensors that makes measurement difficult. Typically, only pressures within the pump are available. Depending on the setup, external measurements may be available. Determining which of 
the limited sensors are necessary to track flow loss is crucial to achieving a sensor system that is practically and economically feasible.

3. Track axial piston pump flow loss over time. As the pump degrades, flow loss is expected to go up. Degrading the pump in the lab is difficult. However, a line has been introduced in the discharge pump volume that goes back to tank to create extra flow losses. A manually operated valve has been put on this line to vary the flow through this path. The goal is to track the changing flow loss through this valve which simulates a real flow loss.

4. Evaluate different filtering methods for tracking flow loss to determine best and most practical filter implementation. There are many different filtering estimation techniques. There are linear filters, like the Kalman filter and pole placement filter, nonlinear filters like the particle filter and unscented filter, and combination of linear and nonlinear systems like the extended Kalman filter. The nonlinear filters often have estimation improvements over the linear filters, typically at the cost of a longer run time. An investigation needs to be done to determine which filters are sufficient to accurately estimate the flow loss.

\subsection{Contributions}

1. Demonstrated that a simple model is sufficient for modeling the discharge pressure of an axial piston pump with flow loss that can be feasibly utilized in an industry setting due to low sample rate and step time. A first order model for the discharge pressure was developed and fit well with experimental data, including dynamic flow loss parameters. 
2. Developed a method of utilizing said simple model in multiple filtering algorithms to track experimental flow time loss changes. This work has demonstrated that a first order model can be implemented in a stochastic filter with one parameter estimation to track flow loss changes with low estimation error. This has been demonstrated with the extended Kalman filter extensively, but also shows the unscented Kalman filter has a similar performance. Furthermore, the Kalman filter and pole placement filter show the ability to detect flow loss changes.

3. Filtering methods demonstrated in this work are filtering methods that are traditionally utilized in industry application, allowing this work to be applicable to more than a laboratory setting. Results show that the extended Kalman filter is a good choice for estimating flow losses. The possible benefits of the particle filter and unscented Kalman filter did not improve estimation. The extended Kalman filter has been an industry standard and is a reasonable filter for out of the laboratory implementation.

\subsection{Overview of the Paper}

Chapter 1 describes the purpose of the axial piston pump, its importance in high uptime, and work others have done for condition monitoring of various systems. Chapter 2 outlines three different models utilized in this work: a first order system, a second order system, and an eighth order system. Chapter 3 discusses different algorithms for estimating flow loss parameters with the focus being on stochastic filtering techniques. Chapter 4 covers the model simulation results of the high order model. Chapter 5 details the 
experimental setup for testing done in the laboratory. Chapter 6 presents the results of the experimental data. The final chapter, Chapter 7, summarizes the findings of this work. 


\section{CHAPTER 2: MODELING}

\subsection{Flow Loss Model}

The goal of this work is to estimate flow losses for an axial piston pump. Choosing an appropriate model for the flow losses is essential. Flow losses can occur in several ways for a pump. There are designed leakage paths within an axial piston pump to help lubricate the components, which is drained through a port known as the case drain. Flow can be loss through the inlet port as well. Compressibility of the fluid is a flow loss. Some flow is temporarily diverted from the discharge flow to adjust the swash plate angle when needed. Categorizing all the flow losses by Reynolds number is a simpler method than modeling each flow loss individually, and has been shown to be an effective way to estimate flow losses [24].

Two types of flow loss have been considered: low Reynolds number flow loss and high Reynolds number flow loss. Low Reynolds number flow loss, $Q_{\text {Low }}$, is defined as flow which has a Reynolds number much less than 1, and is proportional to the pressure, $P$, of the fluid, or

$$
Q_{\text {Low }}=K_{1} P
$$

High Reynolds number flow loss, $Q_{H i g h}$ is then flow which has a Reynolds number much greater than 1, and is proportional to the square root of the fluid pressure, or

$$
Q_{H i g h}=K_{2} \sqrt{P}
$$

Flow loss is only being considered for the discharge pressure volume, so the flow loss, $Q_{L}$, of interest can be written as 


$$
Q_{L}=K_{1} P_{D}+K_{2} \sqrt{P_{D}}
$$

where $P_{D}$ is the discharge pressure. Under nominal operating conditions, the flow loss paths are assumed to be constant area. If this is true, then $K_{1}$ and $K_{2}$ would stay constant - the coefficients are a lumping of constants and area through which the flow is loss. Therefore, estimating $K_{1}$ and $K_{2}$ to see if these values increase would indicate the pump has undergone some degradation leading to increased flow loss. Note that since $Q_{L}$ is a function of $P_{D}$, which is often changing, simply estimating $Q_{L}$ would not be sufficient. Nominal flow loss for an axial piston pump is not constant.

Section 3.7 later discusses some complications with estimating both $K_{1}$ and $K_{2}$ at the same time. Typically, only 1 of the 2 parameters were estimated at a time. Two other flow loss models were considered to help compensate for this difficulty, shown in Eqs. (4) and (5),

$$
\begin{aligned}
& Q_{L}=K_{3}\left(K_{1,0} P_{D}+K_{2,0} \sqrt{P_{D}}\right), \text { and } \\
& Q_{L}=K_{1,0} P_{D}+K_{2,0} \sqrt{P_{D}}+K_{4} P_{D, 0},
\end{aligned}
$$

where $K_{3}$ is interpreted as a gain to scale some nominal value of $K_{1}$ and $K_{2} . K_{4}$ is an offset that would be expected to be zero under nominal conditions, and only be nonzero as pump degradation occurs. A final consideration was to consider the flow loss as all high Reynolds number flow loss, or

$$
Q_{L}=K_{2} \sqrt{P_{D}}
$$




\subsection{Hydraulic System}

The hydraulic system for this work is complex. Several models have been considered for the hydraulic system. Hydraulic diagrams for each of these models can be seen in Figs 6, 7, 3, and 10 in this chapter. The pump is driven by a 200-horsepower electric motor, shown in Figs. 1 and 2.

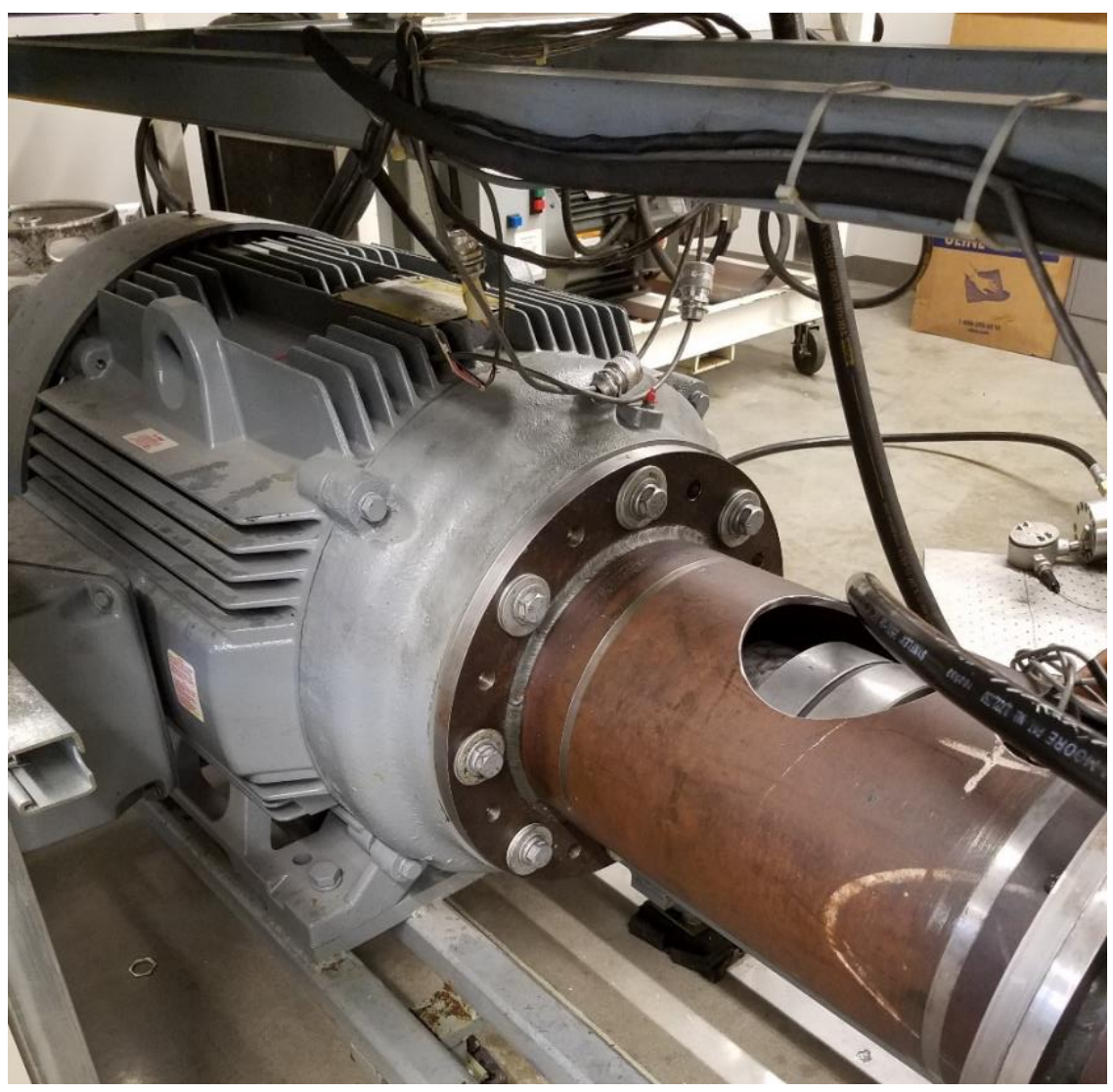

Figure 1. Electric motor. 


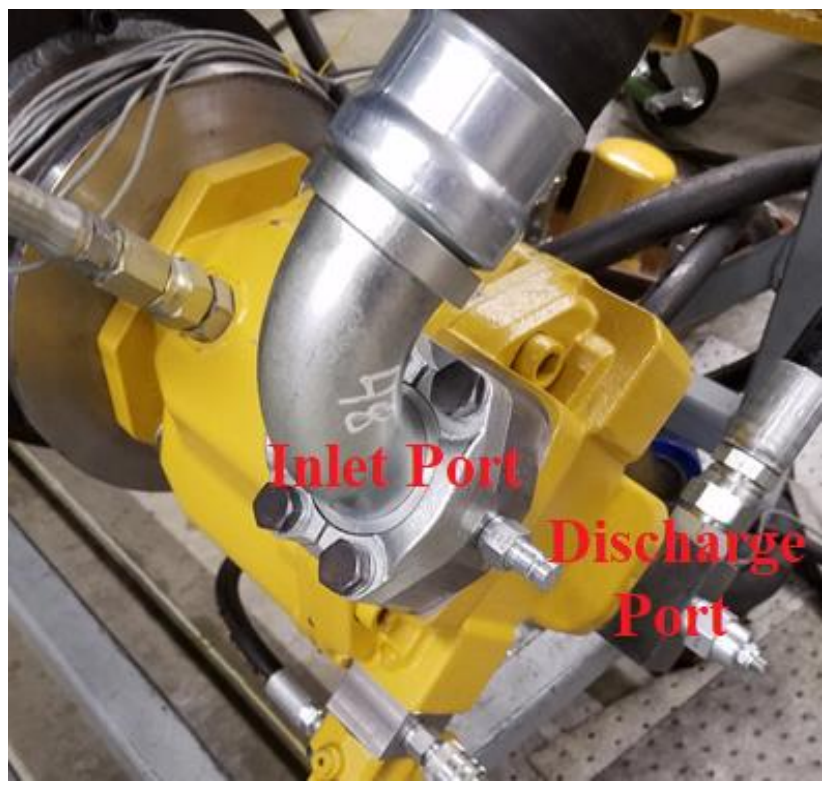

Figure 2. Inlet and discharge port.

The discharge flow line is connected to a flow meter to measure the discharge flow, and then connected to a steering system, shown in Fig 3.

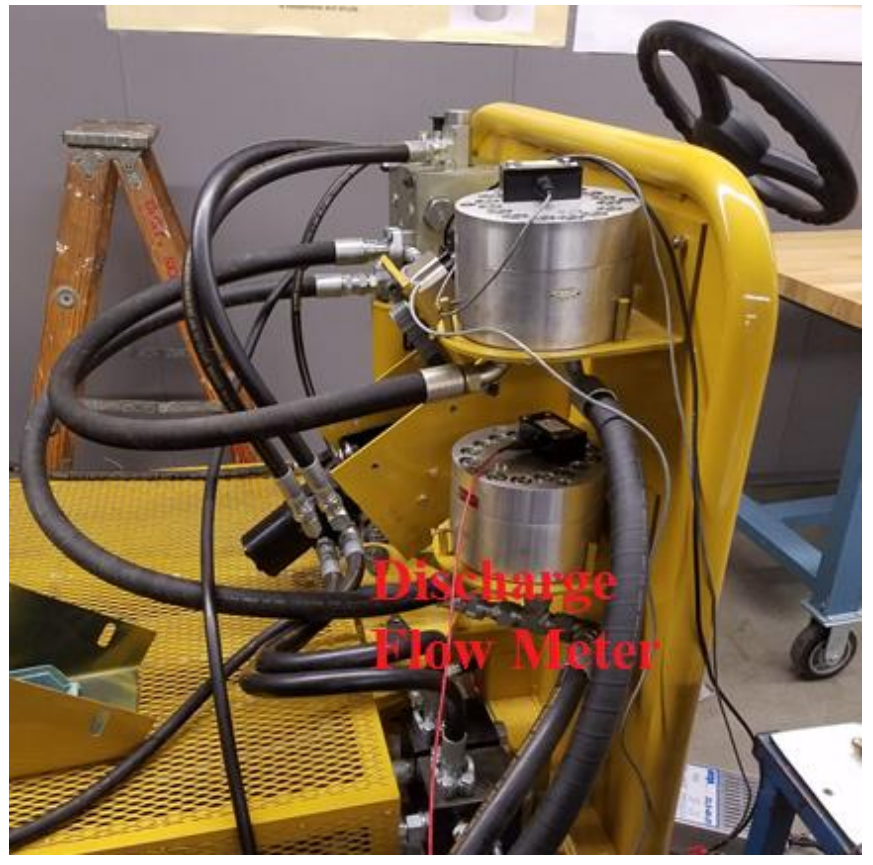

Figure 3. Discharge flow meter and steering system. 
An additional hydraulic line has been added to the discharge line prior to the flow meter that connects to a manually operated needle valve. The flow through this needle valve is measured and sent directly back to the reservoir. This valve is the flow loss valve used to create flow losses within the pump. The valve is shown in Fig. 4.

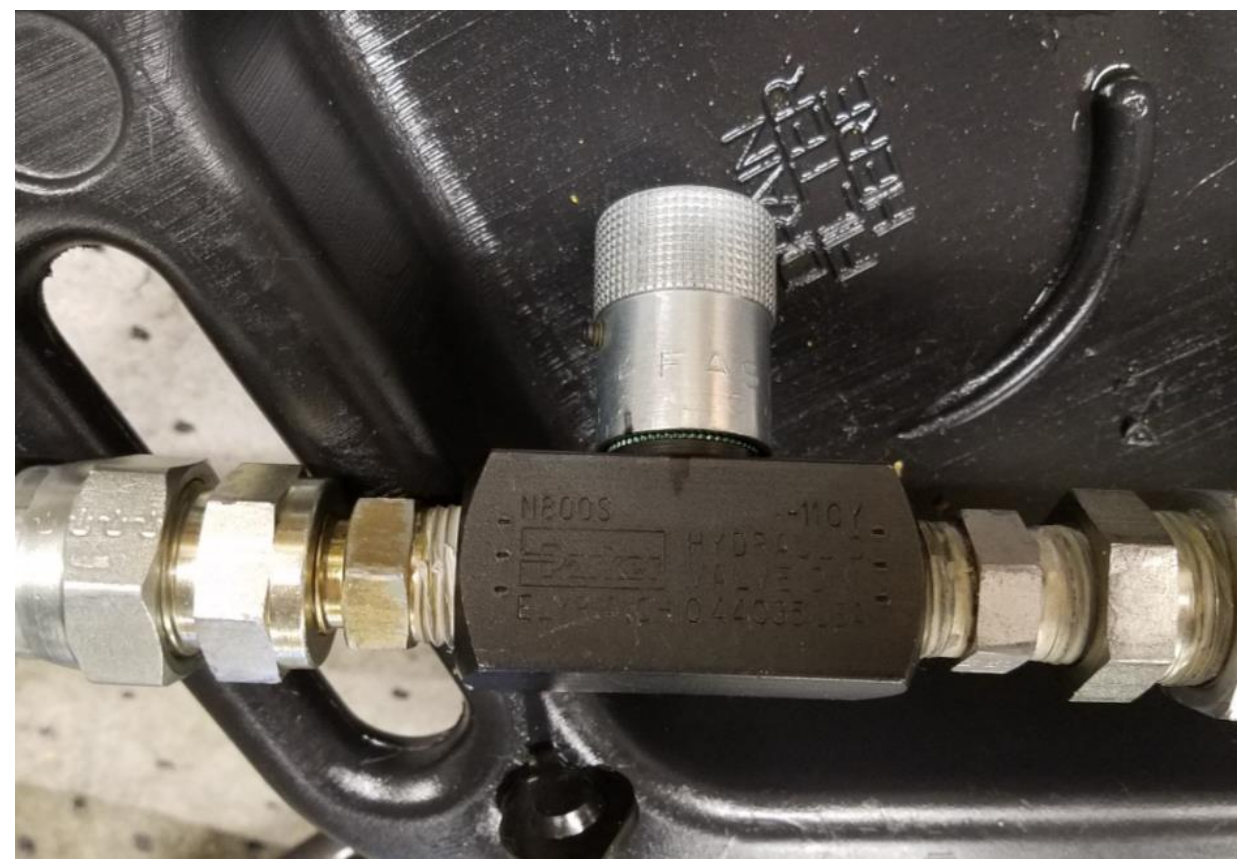

Figure 4. Flow loss manually operated needle valve.

The steering system comes from an off-highway machine, a large haul truck for use in mines and quarries. Flow is controlled by the steering system. The steering wheel is connected to a valve that is hydraulically actuated by turning the wheel. The steering valve opening is proportional to the steering wheel angular velocity. The flow through the valve goes into one of two chambers in a two cylinder-piston linkage to actuate the pistons, shown in Fig. 5. 


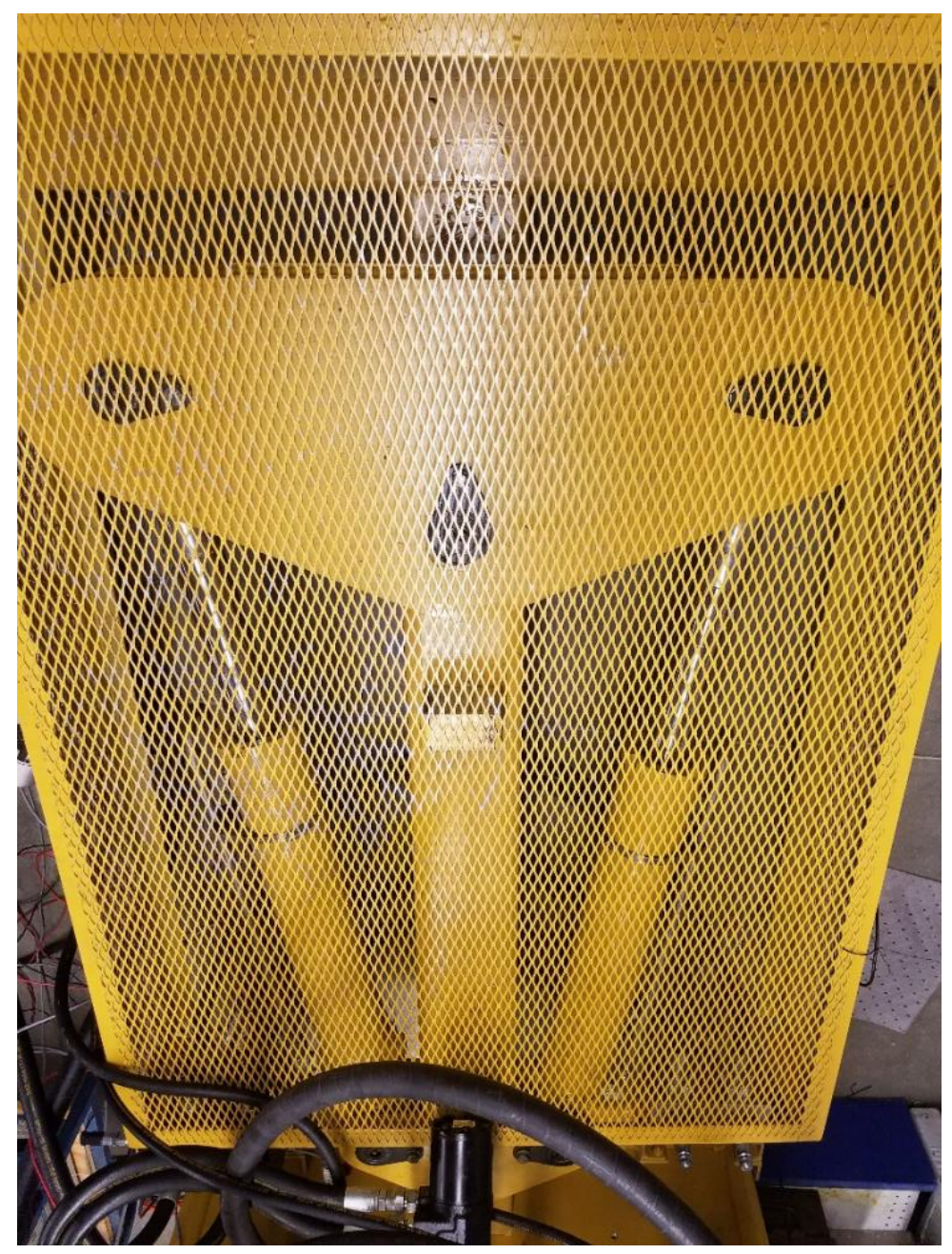

Figure 5. Two cylinder-piston linkage system.

The load pressure of the cylinders is fed back to the pump. A three-way spool valve that is balanced by the load and discharge pressure can shift, depending on the pressure imbalance, to increase or decrease the pump swash plate angle. The swash plate is a mechanism inside the pump that determines the volumetric displacement and therefore how much flow comes from the pump at a given drive speed. The larger the swash plate angle, the more discharge flow there is. One of the goals was to determine how much of the system needed to be modeled to effectively monitor the pump performance, and thus various models were considered including first, second, and eighth order models. 


\subsubsection{First Order Model}

A simple, first order model of the pump discharge pressure was developed. The hydraulic circuit for this model is shown in Fig. 6.

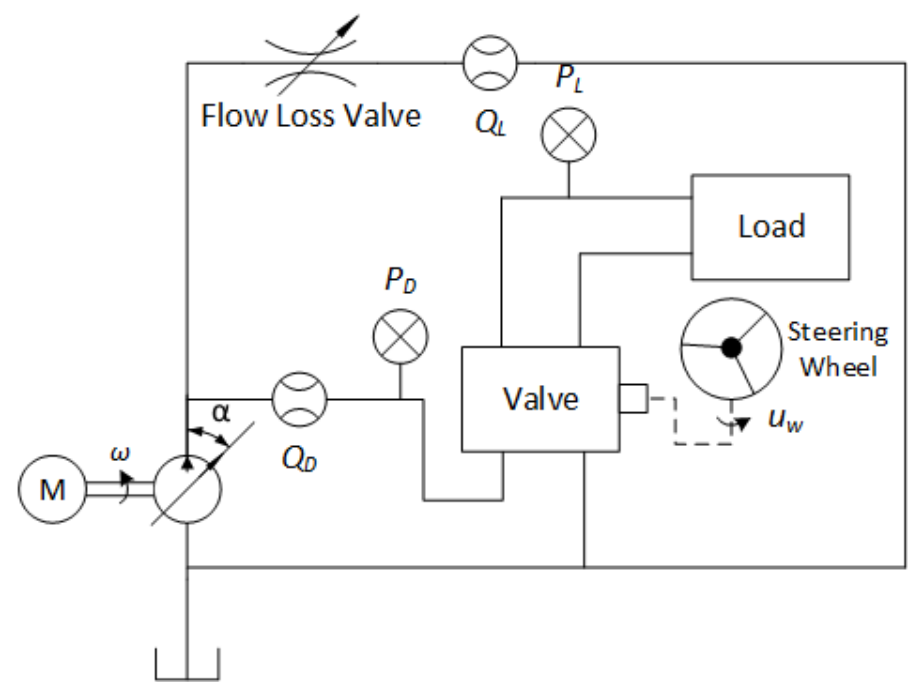

Figure 6. Hydraulic circuit for pump discharge pressure model.

The model for the hydraulic circuit is shown in Eq. (7),

$$
\dot{P}_{D}=\frac{\beta}{V_{d}}\left(Q_{P}-Q_{D}-Q_{L}\right)
$$

where $P_{D}$ is the discharge pressure, $\beta$ is the bulk modulus of the oil, $V_{d}$ is the discharge pressure volume, $Q_{p}$ is the flow coming from the pump, $Q_{D}$ is the flow through the steering valve, and $Q_{L}$ is the total flow loss of the pump. Given the max volumetric displacement per revolution of the pump, $V_{p}$, the motor speed driving the pump, $\omega_{m}$, swash plate angle, $\alpha$, and the maximum swash plate angle, $Q_{P}$ can be determined as

$$
Q_{P}=V_{p} \omega_{m} \frac{\alpha}{\alpha_{\max }}
$$


The orifice equation can be utilized to determined $Q_{D}$. A general form of the orifice equation is

$$
Q=C_{d} A_{v} x \sqrt{\frac{2}{\rho}|\Delta P|} \operatorname{sign}(\Delta P),
$$

where $Q$ is the flow across the valve, $C_{d}$ is the discharge coefficient, $A_{v}$ is the crosssectional area of the valve, $x$ is the valve displacement, $\rho$ is the fluid density, and $\Delta P$ is the pressure differential across the valve. The pressure differential for this system is $P_{D}$ and the load pressure, $P_{L} \cdot P_{D}$ is assumed to be greater than $P_{L}$ always. While the valve position is not known, the steering wheel velocity, $u_{w}$, is and is assumed to be linear with respect to the steering wheel velocity. The constants can be lumped, and $Q_{D}$ can be written as

$$
Q_{D}=K_{w}\left(u_{w}+w_{0}\right) \sqrt{P_{D}-P_{L}},
$$

where $K_{w}$ and $w_{0}$ determine the relationship between the steering speed and the valve position. These values were found experimentally and are presented in section 2.4. Combining Eqs. (3), (7), (8), and (10), the discharge pressure differential equation is

$$
\dot{P}_{D}=\frac{\beta}{V_{d}}\left(V_{p} \omega_{m} \frac{\alpha}{\alpha_{\max }}-K_{w}\left(u_{w}+w_{0}\right) \sqrt{P_{D}-P_{L}}-\left(K_{1} P_{D}+K_{2} \sqrt{P_{D}}\right)\right) .
$$

The single state is $P_{D}$. The inputs to the model are $\alpha, u_{w}$, and $P_{L}$. Note that much of this work is done with a linearized, discrete model in state space form, or

$$
\begin{gathered}
x_{k+1}=A_{k} x_{k}+B_{k} u_{k} \text { and } \\
y_{k}=C_{k} x_{k}+D_{k} u_{k} .
\end{gathered}
$$

For the first order system, $\boldsymbol{A}_{\boldsymbol{k}}$ and $\boldsymbol{B}_{\boldsymbol{k}}$ are

$$
\boldsymbol{A}_{\boldsymbol{k}}=1+h\left(\frac{\beta}{\mathrm{V}_{\mathrm{d}}}\left(-\frac{K_{w}\left(u_{w}^{*}+w_{0}\right)}{2 \sqrt{P_{D}^{*}-P_{L}^{*}}}-K_{1}-\frac{K_{2}}{\sqrt{P_{D}^{*}}}\right)\right) \text { and }
$$




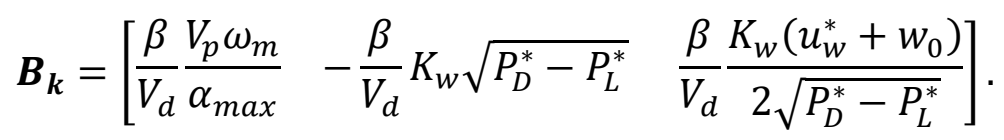

The * indicates the local linearization point, and $h$ is the step size. The output equation, $\boldsymbol{y}_{\boldsymbol{k}}$, is only relevant to filter measurements, and so will be discussed more in the next chapter.

\subsubsection{Second Order Model}

One of the drawbacks of the first order model is that the swash plate, steering wheel, and load dynamics are solely inputs to the model. In order to utilize this model, these inputs still have to be measured from the system. The disadvantage being that all measurements are noisy. One of the benefits of utilizing methods like the KF, which is discussed in greater detail in Chapter 3, is the filtering of noisy measurements to estimate the true state. If these three measurements are only considered inputs to the model, then they are not filtered by the KF. Therefore, modeling these dynamics may offer some benefit.

Of the three inputs, the swash plate angle was chosen to be modeled. The load pressure was not modeled because ideally, estimating flow losses would be independent of whatever load dynamics are present. This independence would make this method of estimation much more general, and therefore applicable to other systems. The steering wheel dynamics was not modeled due to difficulty in determining key parameters necessary in modeling the steering wheel. Of the three inputs, swash plate was the easiest to develop a working model. The hydraulic circuit from Fig. 6 has been expanded in Fig. 7 with detail necessary to model the swash plate. 


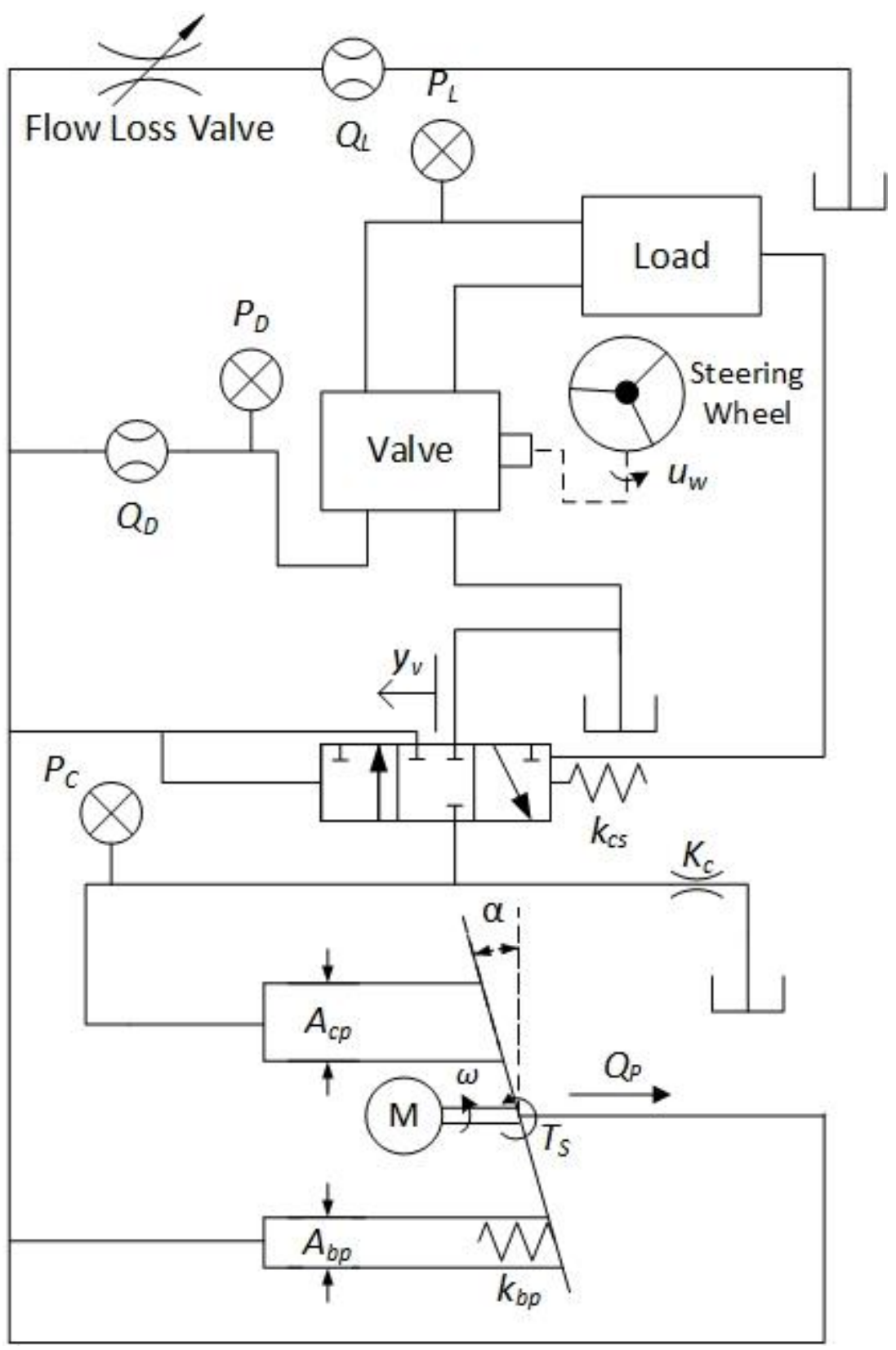

Figure 7. Hydraulic circuit for discharge pressure and swash plate model.

The torque balance on the swash plate is given in Eq. (16),

$$
\ddot{\alpha}=\frac{1}{J}\left(\left(P_{D} A_{b p}-P_{C} A_{c p}+k_{b p} L_{2 a}\left(\tan \alpha_{m a x}-\tan \alpha+x_{0}\right)\right) L_{2 a}-T_{S}\right)
$$

where $J$ is the angular moment of inertia, $A_{b p}$ is the cross-sectional area of the bias piston, $A_{c p}$ is the cross-sectional area of the control piston, $k_{b p}$ is the bias spring rate, $L_{2 a}$ is the 
length from the center of rotation to the piston pitch radius, $x_{0}$ is the spring preload distance, and $T_{S}$ is the swivel torque. The swivel torque was obtained from [25], and is written as

$$
T_{S}=C_{p} P_{D}-C_{i} \alpha
$$

where $C_{p}$ and $C_{i}$ is

$$
\begin{gathered}
C_{p}=\frac{N_{p}}{2} A_{p} r \frac{\gamma}{\pi} \text { and } \\
C_{i}=\frac{N_{p}}{2} m_{p s} r^{2} \omega_{m}^{2}
\end{gathered}
$$

respectively. $N_{p}$ is the number of pistons, $A_{p}$ is the cross-sectional area of the pistons, $r$ is the piston pitch radius, $\gamma$ is this piston passover angle, and $m_{p s}$ is the mass of the piston and slipper.

As can be seen from Eq. (16), $P_{C}$ is necessary to model the dynamics of the swash plate. A model for $P_{C}$ is given as

$$
\dot{P}_{C}=\frac{\beta}{V_{C}}\left(Q_{C}-Q_{L, C}-\dot{V}_{C}\right),
$$

where $V_{C}$ is the control pressure volume, $Q_{C}$ is the flow across the three-way spool valve, and $Q_{L, C}$ is the flow loss of the control volume. $Q_{C}$ depends on the position of the spool valve. If the spool valve is shifted in one direction, the flow goes from the discharge volume to the control volume, decreasing the swash plate position which has a potentially high pressure differential. If the valve is shifted in the other direction, the flow goes from the control pressure back to the reservoir which has a lower pressure differential than when shifted the other direction. If the valve is at its null position, the flow is zero. Instead, of modeling the flow depending on which way the valve is shifted, the flow was assumed to 
be proportional to the valve opening and independent of the pressure drop across the spool valve, or

$$
Q_{C}=-K_{q} y_{v}
$$

where $K_{q}$ was determined through model validation and $y_{v}$ is the spool valve position.

$Q_{L, C}$ is the flow loss of the control pressure. There is a constant area in the control volume that flow is continuously loss through. This area is a design choice because flow loss help dampen out oscillations in the system. The flow loss was modeled as

$$
Q_{L, C}=K_{C} \sqrt{P_{C}}
$$

where $K_{C}$ is a lumping of constants from the orifice equation. Several older pumps were provided for this work. One was disassembled and this flow loss port size was measured to determine $K_{C}$. The area of the port was $0.44 \mathrm{~mm}^{2}$.

The control pressure volume change is due to the change in swash plate position. Assuming a small angle for $\alpha$, the control volume change is

$$
\dot{V}_{C}=-A_{c p} L_{2 a} \dot{\alpha}
$$

Substituting Eqs. (21)-(23) into (20) yields

$$
\dot{P}_{C}=\frac{\beta}{V_{c}}\left(-K_{q} y_{v}-K_{C} \sqrt{P_{C}}+A_{c p} L_{2 a} \dot{\alpha}\right) .
$$

As can be seen from Eq. (24), the control pressure is a function of the three-way spool valve position. The spool valve can be modeled as

$$
\ddot{y}_{v}=\frac{1}{m}\left(-k_{c s} y_{v}-b_{y} \dot{y}_{v}-\left(P_{D}-\left(P_{L}+P_{M}\right)\right) A_{c s o}\right)
$$

where $m$ is the mass of the spool, $k_{c s}$ is the spring rate of the control spring, $b_{y}$ is the viscous friction coefficient, $A_{c s o}$ is the diameter of the spool valve, and $P_{M}$ is the margin pressure. Note that the margin pressure is not an actual force. The spring is given a preload 
that has an equal force as $P_{M} A_{c s o}$. Omitting $P_{M}$ and including a spring preload distance would be an equivalent system.

Recall that the swash plate was modeled to determine if the KF could improve estimation by filtering the swash plate measurement. Consequently, both control pressure and the control valve dynamics needed to be modeled. Instead of going from a first order system to a sixth order system, a steady state analysis was done for several states. By setting $\dot{y}_{v}$ and $\ddot{y}_{v}$ to zero, the steady state control valve position can be calculated as

$$
y_{v}=\frac{\left(P_{L}+P_{M}-P_{D}\right) A_{c s o}}{k_{c s}}
$$

The control pressure and swash plate dynamics were also simplified. By setting $\ddot{\alpha}$ to zero, Eq. (16) could be rearranged to

$$
P_{C}=\frac{1}{A_{c p} L_{2 a}}\left(\left(A_{b p} L_{2 a}-C_{p}\right) P_{D}+\left(C_{i}-k_{b p} L_{2 a}^{2}\right) \alpha+k_{b p} L_{2 a}^{2}\left(\alpha_{\max }+x_{0}\right)\right) .
$$

Setting $\dot{P}_{C}$ to zero and rearranging yields

$$
\dot{\alpha}=\frac{1}{A_{c p} L_{2 a}}\left(K_{q} y_{v}+K_{C} \sqrt{P_{C}}\right) .
$$

The second order model is presented in Eqs. (29) and (30). Note that Eq. (29) is the same as Eq. (11), and is repeated solely as a reminder. Equation (30) was derived by substituting Eqs. (26) and (27) into (28). The two equations are

$$
\begin{gathered}
\dot{P}_{D}=\frac{\beta}{V_{d}}\left(V_{p} \omega_{m} \frac{\alpha}{\alpha_{\max }}-K_{w}\left(u_{w}+w_{0}\right) \sqrt{P_{D}-P_{L}}-\left(K_{1} P_{D}+K_{2} \sqrt{P_{D}}\right)\right) \text { and } \\
\dot{\alpha}=\frac{1}{A_{c p} L_{2 a}}\left(\frac{K_{q}\left(P_{L}+P_{M}-P_{D}\right) A_{c s o}}{k_{c s}}\right)+ \\
\frac{K_{c}}{A_{c p} L_{2 a}} \sqrt{\frac{1}{A_{c p} L_{2 a}}\left(\left(A_{b p} L_{2 a}-C_{p}\right) P_{D}+\left(C_{i}-k_{b p} L_{2 a}^{2}\right) \alpha+k_{b p} L_{2 a}^{2}\left(\alpha_{\max }+x_{0}\right)\right)}
\end{gathered}
$$

The linearized, discrete $\boldsymbol{A}_{\boldsymbol{k}}$ and $\boldsymbol{B}_{\boldsymbol{k}}$ matrices are 


$$
\begin{gathered}
\boldsymbol{A}_{\boldsymbol{k}} \\
=\left[\begin{array}{cc}
1+h\left(\frac{\beta}{\mathrm{V}_{\mathrm{d}}}\left(-\frac{K_{w}\left(u_{w}^{*}+w_{0}\right)}{2 \sqrt{P_{D}^{*}-P_{L}^{*}}}-K_{1}-\frac{K_{2}}{2 \sqrt{P_{D}^{*}}}\right)\right) & h\left(\frac{\beta}{V_{d}} \frac{V_{p} \omega_{m}}{\alpha_{m a x}}\right) \\
h\left(\frac{-K_{q} A_{c s o}}{A_{c p} L_{2 a} k_{c s}}+\frac{K_{c}\left(A_{b p} L_{2 a}-C_{p}\right)}{2 A_{c p}^{2} L_{2 a}^{2} \sqrt{C_{1}}}\right. & \left.1+h \frac{K_{c}\left(C_{i}-k_{b p} L_{2 a}^{2}\right)}{2 A_{c p}^{2} L_{2 a}^{2} \sqrt{C_{1}}}\right]
\end{array}\right] \text { and } \\
\boldsymbol{B}_{\boldsymbol{k}}=\left[\begin{array}{ccc}
\frac{\beta}{V_{d} \frac{V_{p} \omega_{m}}{\alpha_{\max }}}-\frac{\beta}{V_{d}} K_{w} \sqrt{P_{D}^{*}-P_{L}^{*}} & \frac{\beta}{V_{d}} \frac{K_{w}\left(u_{w}^{*}+w_{0}\right)}{2 \sqrt{P_{D}^{*}-P_{L}^{*}}} \\
0 & \frac{K_{q} A_{c s o}}{A_{c p} L_{2 a} k_{c s}}
\end{array}\right]
\end{gathered}
$$

where $C_{1}$ is

$$
C_{1}=\frac{1}{A_{c p} L_{2 a}}\left(\left(A_{b p} L_{2 a}-C_{p}\right) P_{D}^{*}+\left(C_{i}-k_{b p} L_{2 a}^{2}\right) \alpha^{*}+k_{b p} L_{2 a}^{2}\left(\alpha_{\max }+x_{0}\right)\right)
$$

\subsubsection{Eighth Order Model}

Although the bulk of this work focuses on the first and second order model, an eighth order model was also utilized. The high fidelity, eighth order model was developed and validated prior to this work for a very similar system and can be seen in much greater detail in [26]. The hydraulic circuit for this model is shown in Fig. 8. 


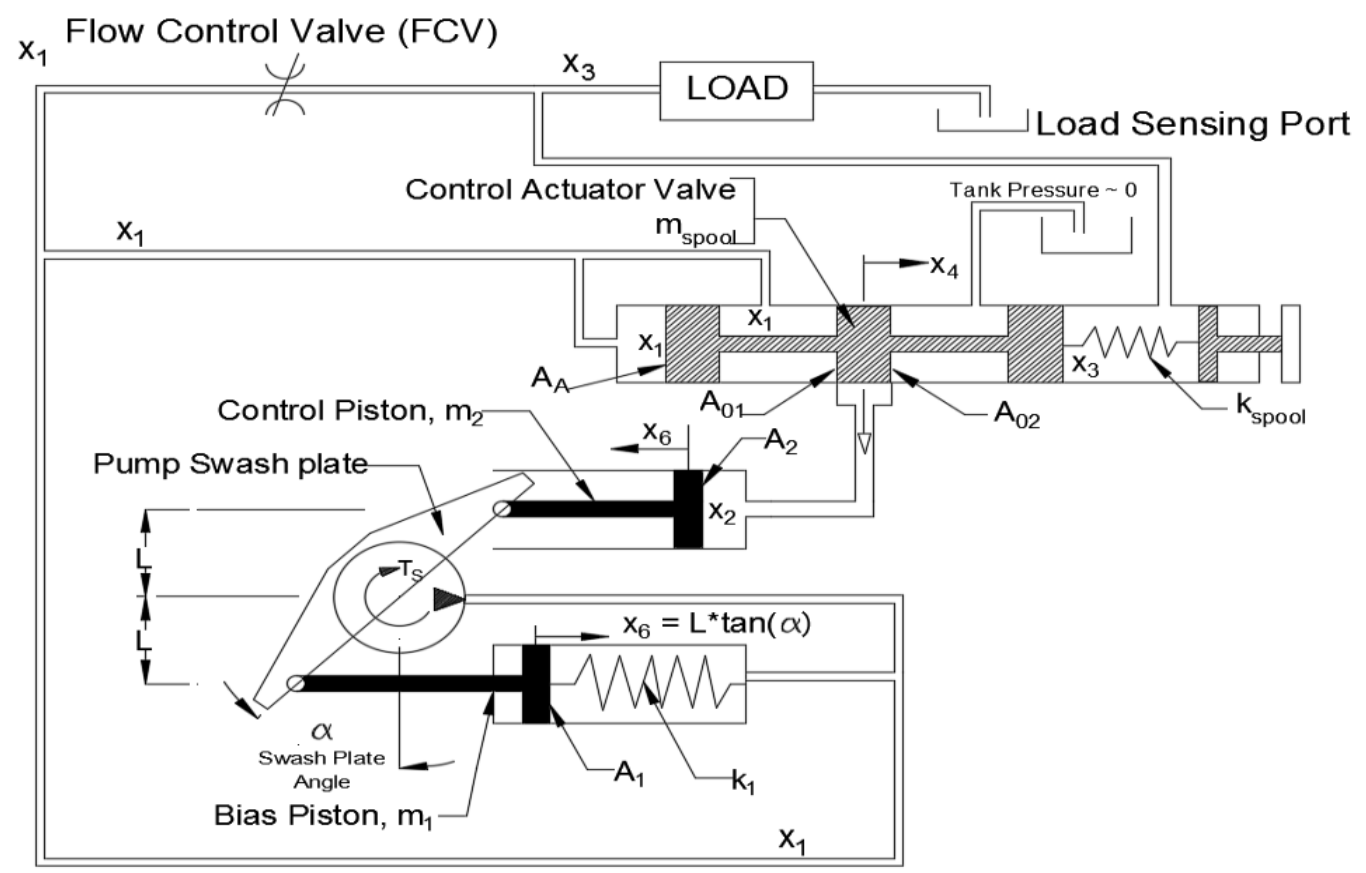

Figure 8. Hydraulic circuit for high fidelity model.

The single input to the model is the flow control valve. This valve is analogous to the steering wheel valve in the previous models. The states for this model are

$$
\boldsymbol{x}=\left[\begin{array}{l}
x_{1} \\
x_{2} \\
x_{3} \\
x_{4} \\
x_{5} \\
x_{6} \\
x_{7} \\
x_{8}
\end{array}\right]=\left[\begin{array}{c}
\text { Discharge Pressure } \\
\text { Control Pressure } \\
\text { Load Pressure } \\
\text { Control Spool Position } \\
\text { Control Spool Velocity } \\
\text { Control Piston Position } \\
\text { Control Piston Velocity } \\
\text { Motor Load Velocity }
\end{array}\right]
$$

The eight differential equations for this model are

$$
\begin{gathered}
\dot{x}_{1}=\frac{\beta}{V_{d}}\left(\frac{V_{p} \alpha}{\alpha_{\max }} \omega_{m}-C_{d} A_{m} u \sqrt{\frac{2}{\rho}\left(x_{1}-x_{3}\right)}-A_{c p} x_{7}\right), \\
\dot{x}_{2}=\frac{\beta}{V_{c}}\left(C_{d} A_{01} \sqrt{\frac{2}{\rho}\left(x_{1}-x_{2}\right)}-C_{d}\left(A_{02}+K_{C}\right) \sqrt{\frac{2}{\rho}\left(x_{2}\right)}-A_{2} x_{7}\right),
\end{gathered}
$$




$$
\begin{gathered}
\dot{x}_{3}=\frac{\beta}{V_{L}}\left(A_{m} C_{d} \sqrt{\frac{2}{\rho}\left(x_{1}-x_{3}\right)}-V_{m} x_{8}\right), \\
\dot{x}_{4}=x_{5}, \\
\dot{x}_{5}=\frac{1}{m_{\text {spool }}}\left(A_{A} x_{1}-k_{s p o o l}\left(x_{4}+x_{p 0}\right)-A_{A} x_{3}-F_{f}-K_{S V} x_{5}\right), \\
\dot{x}_{7}=\frac{1}{m_{1}+m_{2}}\left(A_{2} x_{2}-x_{7},\right. \\
\left.k_{1}\left(x_{6 p}+x_{6}\right)-A_{1} x_{1}-\frac{C_{i} \alpha-C_{p} x_{1}}{L_{1}}-K_{A G} x_{7}\right), \text { and } \\
\dot{x}_{8}=\frac{V_{m} x_{3}-b_{m} x_{8}}{J_{m}} .
\end{gathered}
$$

\subsection{Augmenting Model to Include Parameter Estimation}

Augmentation of the model to include parameter estimation is a straight forward change [27]. For each parameter being estimated, an extra state is appended to the model with zero dynamics. As an example, if the first order model was to include $K_{2}$ to be estimated, then a second state equation would be included as $\dot{K}_{2}=0$. Note that this augmentation is only useful for utilizing these models in the filter algorithms, as simulating a state with zero dynamics is redundant.

Parameter estimation does cause the model to no longer be stable. In the continuous time domain, a pole would clearly be at the origin and therefore the system is marginally stable due to the parameter state. This marginal stability is problematic for the observability analysis, to be discussed later in section 3.7. Note that throughout this text, the models are referred to as "first", "second", and "eighth". These are in reference to the models as they were presented without parameter states appended to the models. In the results section, if 
the first order model is being utilized and a parameter is being estimated, the model is still referred to as the first order model, despite technically being second order due to the parameter state.

\subsection{Discharge Flow Model Validation}

Since the steering wheel valve opening cannot be measured, its position must be determined from the steering wheel velocity. The valve is actuated by a steering system; the faster the steering system turns the wider the valve opening is. Steering velocity is measured, so flow across the steering wheel valve is calculated using Eq. (9). A set of experiments to vary the pressures and flow was done to determine $K_{w}$ and $w o$. Figure 9 shows the results of these experiments, with the square root pressure difference moved to the left side of the equation.

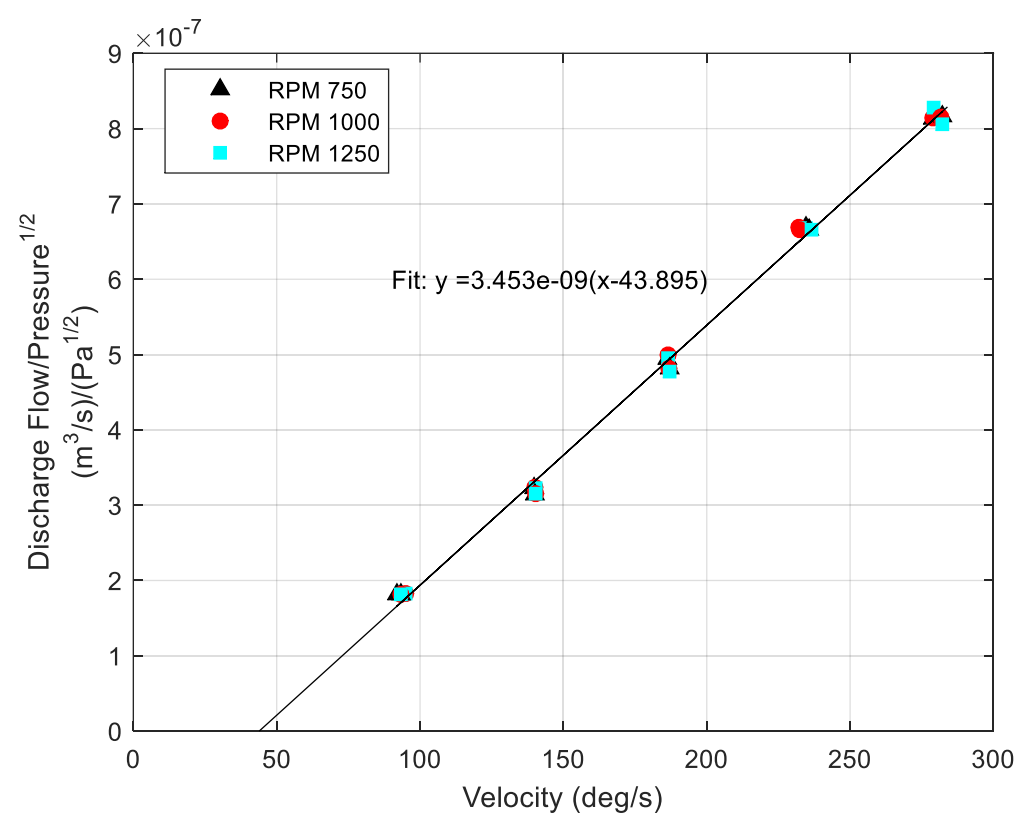

Figure 9. Steering wheel valve flow validation. 
There is an offset in the fit, but clearly if the steering wheel is not moving, there is no flow. There is one of two possibilities: the relationship at low velocities is nonlinear, or there is a certain velocity threshold for which the valve will not open until the threshold is met. However, this discrepancy is at very low turning speeds and so can be neglected. Under certain circumstances, this model was found to be inadequate. When the swash plate is at maximum position, the flow model underestimates the flow through the valve. The steering wheel is a pump itself. It is believed that much of the flow is going through the gerotor pump in the steering wheel at maximum swash plate angle, explaining why the valve could be open less than expected. Regardless, a better flow model is important to accurately estimate the flow losses. Another way to estimate the flow across the steering wheel valve is to calculate the rate of volume changes in the two cylinders that the discharge flow actuates. A diagram of the two-cylinder system is presented in Fig. 10. 


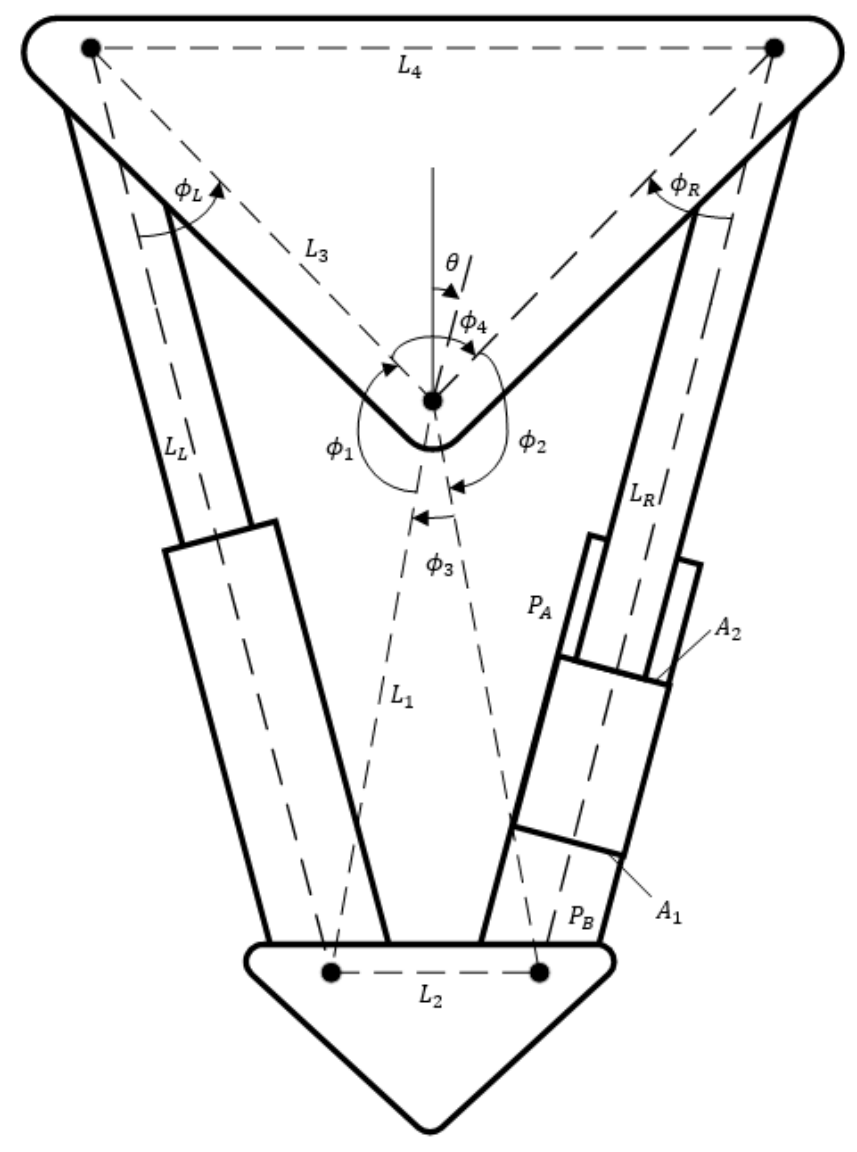

Figure 10. Two-cylinder load diagram.

The sum of the torques about the pivot point of the wedge is

$$
\ddot{\theta}=\frac{1}{J_{w}}\left(\left(P_{A} A_{1}-P_{B} A_{2}\right) L_{3} \sin \left(\phi_{L}\right)-\left(P_{B} A_{1}-P_{A} A_{2}\right) L_{3} \sin \left(\phi_{R}\right)\right)
$$

Flow either goes into chamber A or chamber B, depending on the steering direction. The area of the pistons is known, so the flow rate can be determined from the velocity of the pistons multiplied by the cross-sectional area of the pistons.

Inverse law of cosines can be used to determine $\phi_{L}$ and $\phi_{R}$ :

$$
\phi_{L}=\cos ^{-1}\left(\frac{L_{3}^{2}+L_{L}^{2}-L_{1}^{2}}{2 L_{3} L_{L}}\right) \text { and }
$$




$$
\phi_{R}=\cos ^{-1}\left(\frac{L_{3}^{2}+L_{R}^{2}-L_{1}^{2}}{2 L_{3} L_{R}}\right)
$$

The length of the left and right cylinders, $L_{L}$ and $L_{R}$, respectively, can be determined with the law of cosines,

$$
\begin{gathered}
L_{L}=\sqrt{L_{3}^{2}+L_{1}^{2}-2 L_{3} L_{1} \cos \left(\phi_{1}\right)} \text { and } \\
L_{R}=\sqrt{L_{3}^{2}+L_{1}^{2}-2 L_{3} L_{1} \cos \left(\phi_{2}\right)} .
\end{gathered}
$$

Note that $\theta=0.5\left(\phi_{1}-\phi_{2}\right)$ and $\phi_{1}+\phi_{2}+\phi_{3}+\phi_{4}=2 \pi$. Therefore, Eqs. (46) and (47) can be rewritten as

$$
\begin{gathered}
L_{L}=\sqrt{L_{3}^{2}+L_{1}^{2}-2 L_{3} L_{1} \cos \left(\theta+\pi-\frac{1}{2} \phi_{3}-\frac{1}{2} \phi_{4}\right)} \text { and } \\
L_{R}=\sqrt{L_{3}^{2}+L_{1}^{2}-2 L_{3} L_{1} \cos \left(-\theta+\pi-\frac{1}{2} \phi_{3}-\frac{1}{2} \phi_{4}\right)} .
\end{gathered}
$$

where

$$
\begin{gathered}
\phi_{3}=\cos ^{-1}\left(\frac{2 L_{1}^{2}-L_{4}^{2}}{2 L_{1}^{2}}\right) \text { and } \\
\phi_{4}=\cos ^{-1}\left(\frac{2 L_{3}^{2}-L_{4}^{2}}{2 L_{3}^{2}}\right) .
\end{gathered}
$$

The velocities of the two cylinders can be determined by differentiation, and the cross sections are known for the rod and head end. The flow across the steering wheel valve can then be estimated as

$$
Q_{D, e s t}=\left\{\begin{array}{l}
\left|\dot{L}_{L}\right| A_{1}+\left|\dot{L}_{R}\right| A_{2} \text { for } \dot{L}_{L}>0 \\
\left|\dot{L}_{L}\right| A_{2}+\left|\dot{L}_{R}\right| A_{1} \text { for } \dot{L}_{L}<0
\end{array} .\right.
$$




\section{CHAPTER 3: FLOW LOSS ESTIMATION TECHNIQUES}

\subsection{General Least Squares Fit}

One way to determine the flow loss coefficients would be to collect a large set of data and do a general least squares fit [28]. Consider the volumetric efficiency, $\eta_{V}$, of the pump, written as

$$
\eta_{V}=\frac{Q_{D}}{Q_{P}}=\frac{Q_{P}-Q_{L}}{Q_{P}}=1-\frac{1}{Q_{p}}\left(K_{1} P_{D}+K_{2} \sqrt{P_{D}}\right)
$$

$Q_{D}$ is easily measured in a testing condition, $Q_{P}$ is determined from the swash plate angle measurement, and $P_{D}$ is also measured. The least squares fit is

$$
\left[\begin{array}{l}
K_{1} \\
K_{2}
\end{array}\right]=\left(\boldsymbol{Z}^{T} \boldsymbol{Z}\right)^{-1}\left(\boldsymbol{Z}^{T} \boldsymbol{Y}\right)
$$

where $\boldsymbol{Z}$ is

$$
\boldsymbol{Z}=\left[\begin{array}{ll}
\boldsymbol{P}_{\boldsymbol{D}} \frac{\alpha_{\max }}{V_{p} \boldsymbol{\omega}_{m} \boldsymbol{\alpha}} & \sqrt{\boldsymbol{P}_{\boldsymbol{D}}} \frac{\alpha_{\max }}{V_{p} \boldsymbol{\omega}_{m} \boldsymbol{\alpha}}
\end{array}\right]
$$

and $\boldsymbol{Y}$ is

$$
\boldsymbol{Y}=1-\boldsymbol{\eta}_{\boldsymbol{V}}
$$

$\boldsymbol{P}_{\boldsymbol{D}}, \boldsymbol{\omega}_{\boldsymbol{m}}, \boldsymbol{\alpha}$, and $\boldsymbol{\eta}_{\boldsymbol{V}}$ are $m \times 1$ vectors, where $m$ is the number of data points collected. $\boldsymbol{Y}$ is also $m \times 1$ and $\boldsymbol{Z}$ is $m \times p$, where $p$ is the number of coefficients being fit. The general least squares method does have some advantages. The calculation is both simple and is computationally efficient. Note that while Eq. (44) does have a matrix inversion, the matrix is only $2 \times 2$ since $p=2$. There are several disadvantages as well. The efficiency equation assumes steady state. The fit also requires a lot of data at multiple operating points to give a reasonable fit. Therefore, this method could not be utilized to track flow losses in real 
time. As will be shown later, utilizing filters does not require multiple operating points for estimating flow loss.

\subsection{Kalman Filter}

The discrete, linear Kalman filter is a state observer that predicts the states of a linear model, given measurements of a set of the states [29]. Often the measurements will have noise associated with them, assumed to be Gaussian. The KF has several key advantages. In comparison to other filtering techniques, it is one of the simplest algorithms to implement. Because the algorithm is all linear matrix algebra, the KF is easily implemented in real time as well. However, there are several disadvantages to the KF. The measurement and process noise are assumed to be zero mean Gaussian. While there are a lot of scenarios where this is an appropriate assumption, there is a wide range of applications where this is not accurate. For example, electrical noise from sensor measurement is often zero mean Gaussian and is therefore an appropriate situation to make this assumption. However, process noise, or unmodeled dynamics, is often not Gaussian. As a general example, friction forces are often neglected in models. Friction forces typically do not behave a zero mean Gaussian distribution.

Given the linearized, discrete system:

$$
\begin{gathered}
x_{k}=A x_{k-1}+B u_{k-1}+v_{k} \\
y_{k}=C x_{k}+n_{k}
\end{gathered}
$$

where $\boldsymbol{A}$ is the linear state transition matrix, $\boldsymbol{B}$ is the control input matrix, and $\boldsymbol{C}$ is the observation matrix. The Kalman filter works by recursively estimating a posteriori states and uncertainty of those estimates, using known a priori state and uncertainty estimates, 
The newly updated state and uncertainty estimates are then reused to find the a priori estimates of the next time iteration.

The initial $(k=1)$ a priori state estimate, $\hat{x}_{k \mid k-1}$, and estimate covariance, $P_{k \mid k-1}$, is calculated given the discrete system at $t_{k}$ and initial state values, $\hat{x}_{0 \mid 0}$ and $P_{0 \mid 0}$ :

$$
\begin{gathered}
\widehat{x}_{k \mid k-1}=A \widehat{x}_{0 \mid 0}+B u_{0}, \\
P_{k \mid k-1}=A P_{0 \mid 0} A^{T}+Q_{k},
\end{gathered}
$$

where $\boldsymbol{Q}_{\boldsymbol{k}}: \boldsymbol{v}_{\boldsymbol{k}} \sim N\left(0, \boldsymbol{Q}_{\boldsymbol{k}}\right)$. The algorithm then becomes recursive. The current optimal Kalman gain can be calculated using the residual covariance and a priori estimate covariance:

$$
\boldsymbol{K}_{\boldsymbol{k}}=\boldsymbol{P}_{\boldsymbol{k} \mid \boldsymbol{k}-1} \boldsymbol{C}^{T} \times\left(\boldsymbol{C} \boldsymbol{P}_{\boldsymbol{k} \mid \boldsymbol{k}-\mathbf{1}} \boldsymbol{C}^{T}+\boldsymbol{R}_{\boldsymbol{k}}\right)^{-1},
$$

where $\boldsymbol{R}_{\boldsymbol{k}}: \boldsymbol{n}_{\boldsymbol{k}} \sim N\left(0, \boldsymbol{R}_{\boldsymbol{k}}\right)$. With the optimal Kalman gain, the a posteriori state estimates and estimate covariances are:

$$
\begin{gathered}
\widehat{\boldsymbol{x}}_{\boldsymbol{k} \mid \boldsymbol{k}}=\widehat{\boldsymbol{x}}_{\boldsymbol{k} \mid \boldsymbol{k}-1}+K_{k}\left(\boldsymbol{y}_{\boldsymbol{k}}-C \widehat{\boldsymbol{x}}_{\boldsymbol{k} \mid k-1}\right) \\
\boldsymbol{P}_{\boldsymbol{k} \mid \boldsymbol{k}}=\left(I-K_{k} C\right) P_{k \mid k-1}
\end{gathered}
$$

The a priori state estimate and estimate covariance can then be calculated for use in the next iteration:

$$
\begin{gathered}
\widehat{x}_{k \mid k-1}=A \widehat{x}_{k-1 \mid k-1}+B u_{k-1} \\
P_{k \mid k-1}=A P_{k-1 \mid k-1} A^{T}+Q_{k}
\end{gathered}
$$




\subsection{Pole Placement Filter}

The pole placement filter (PPF) is a simplified version of the KF. Instead of updating the Kalman gain and error covariance at each step, the PPF considers a constant gain. The algorithm simplifies to

$$
\begin{gathered}
\widehat{x}_{k \mid k-1}=A \widehat{x}_{k-1 \mid k-1}+B u_{k-1}, \\
\widehat{x}_{k \mid k}=\widehat{x}_{k \mid k-1}+K\left(y_{k}-C \widehat{x}_{k \mid k-1}\right) .
\end{gathered}
$$

The gain can be determined from the place() command in MATLAB. Given the $\mathbf{A}$ and $\mathbf{C}$ matrices from the state space equations, as well as appropriate poles for the speed of response of the observer, the gains can be determined appropriately. Typically, the poles should be faster than that of the system. The PPF is an extremely computationally efficient algorithm. However, besides that it offers little benefit above the KF.

\subsection{Extended Kalman Filter}

The extended Kalman filter (EKF) is similar to the KF, with two exceptions. The prior state estimation propagation is done utilizing the nonlinear model, and the model is relinearized at each step. These two changes allow the nonlinear dynamics to be represented in the state estimation and eliminates the issue of states moving away from the local linearization. The Kalman filter gain update is still based on the linear model however. The EKF algorithm is presented in Eqs. (68) through (73).

$$
\begin{array}{r}
\boldsymbol{A}_{\boldsymbol{k}}=\left.\frac{\partial \boldsymbol{f}(\boldsymbol{x}, \boldsymbol{u})}{\partial x}\right|_{x=x_{k-1 \mid k-1}} \\
\widehat{\boldsymbol{x}}_{\boldsymbol{k} \mid \boldsymbol{k}-1}=\boldsymbol{f}\left(\boldsymbol{x}_{\boldsymbol{k}-1 \mid \boldsymbol{k}-1}, \boldsymbol{u}_{\boldsymbol{k}-1 \mid k-1}\right)
\end{array}
$$




$$
\begin{aligned}
& \boldsymbol{P}_{k \mid k-1}=A_{k} P_{k-1 \mid k-1} A_{k}^{T}+Q_{k} \\
& \boldsymbol{K}_{\boldsymbol{k}}=\boldsymbol{P}_{\boldsymbol{k} \mid \boldsymbol{k}-1} \boldsymbol{C}_{\boldsymbol{k}}^{T} \times\left(\boldsymbol{C}_{\boldsymbol{k}} \boldsymbol{P}_{\boldsymbol{k} \mid \boldsymbol{k}-1} \boldsymbol{C}_{\boldsymbol{k}}^{T}+\boldsymbol{R}_{\boldsymbol{k}}\right)^{-1}, \\
& \widehat{x}_{k \mid k}=\widehat{x}_{k \mid k-1}+K_{k}\left(y_{k}-C_{k} \widehat{x}_{k \mid k-1}\right) \\
& \boldsymbol{P}_{k \mid k}=\left(I-K_{k} C_{k}\right) P_{k \mid k-1}
\end{aligned}
$$

\subsection{Particle Filter}

Particle filters are nonlinear state observers that approximate the posterior state distribution as a set of discrete, weighted samples called particles. In other words, it is a technique for implementing a recursive Bayesian filter using Monte Carlo simulations. Though they are suboptimal, the advantage of particle filters is that they can be applied to systems which may be nonlinear and have non-Gaussian noise terms [30]. As the number of particles are increased, accuracy increases, and the optimal solution is approached. Also, since they are based on probability distributions, they can help manage the uncertainties typically encountered during state estimation. Disadvantages of using particle filters include the need for accurate models of the system including process and observation noise and the need for intensive computational requirements.

Given a nonlinear system described by:

$$
\begin{aligned}
& x_{k}=f_{k}\left(t_{k}, x_{k-1}, u_{k-1}, v_{k-1}\right) \\
& y_{k}=g_{k}\left(t_{k}, x_{k-1}, u_{k-1}, n_{k-1}\right)
\end{aligned}
$$

where $x(t)$ is the state vector, $y(t)$ is the output vector, $t$ is a continuous time variable, $u(t)$ is the input vector, $v(t)$ is the process noise vector, and $n(t)$ is the observation noise vector. This format is meant to be general and place no restrictions on the functional forms. 
The tracking problem is to recursively calculate a probability distribution of the state, $x_{k}$, at time, $t_{k}$, given the data $y_{1: k}$. Then, the probability distribution function (pdf) may be obtained recursively in two stages, prediction then update. At time step, $k$, a new measurement $y_{k}$ becomes available and used to update the prior estimate.

With particle filters, the approximation to the state distribution is given by two sets of particles, a set of particles representing the state estimate and a second set of associated weights, represented by:

$$
\left\{x_{k}^{i}, w_{k}^{i}\right\}_{i}^{N}
$$

where $N$ denotes the number of particles, and for particle $i, x_{k}^{i}$ denotes the state estimate and $w_{k}^{i}$ denotes the associated weight.

The posterior (current) estimate of the state, i.e. the pdf of the state, is then approximated by:

$$
p\left(x_{k} \mid y_{1: k}\right)=\sum_{i=1}^{N} w_{k}^{i} \delta\left(x_{1: k}-x_{1: k}^{i}\right)
$$

The weights are chosen using the principle of importance sampling. Where the samples $x_{1: k}^{i}$ are drawn from a set of representative samples that form an importance density, $q\left(x_{1: k} \mid y_{1: k}\right)$. An associated weight is then calculated using Bayes theorem

$$
w_{k}^{i} \propto w_{k-1}^{i} \frac{p\left(y_{k} \mid x_{k}^{i}\right) p\left(x_{k}^{i} \mid x_{k-1}^{i}\right)}{q\left(x_{k}^{i} \mid x_{k-1}^{i}, y_{k}\right)} .
$$

There are multiple ways to approximate the importance density distribution, $q\left(x_{1: k} \mid y_{1: k}\right)$. If the posterior samples, $x_{1: k}^{i}$, are considered to be drawn from the importance density distribution, then the importance density distribution could be considered to be the 
posterior samples themselves, $x_{1: k}^{i} \sim q\left(x_{1: k} \mid y_{1: k}\right)$. One could also consider the importance density to be the prior samples, $x_{1: k-1}^{i} \sim q\left(x_{1: k} \mid y_{1: k}\right)$, therefore:

$$
q\left(x_{1: k} \mid y_{1: k}\right) \sim p\left(x_{k}^{i} \mid x_{k-1}^{i}\right)
$$

If this is the case, then substituting (79) into (78) yields

$$
w_{k}^{i} \propto w_{k-1}^{i} p\left(y_{k} \mid x_{k}^{i}\right) .
$$

This type of estimation algorithm is known as the bootstrap particle filter. Within every particle filter framework is the resampling feature, the most common and the one applied in this work is the Sequential Importance method.

To reduce degeneracy in the particle distributions, resampling of the particles should take place. The basic idea of resampling is to eliminate particles that have small weights, therefore concentrating on those particles with larger weights. Resampling involves generating a new set, $\left\{x_{k}^{i *}\right\}_{i=1}^{N s}$ from the old set with $N s$ replacements.

An estimate of the degeneracy of the particles is the effective sample size, $N_{\text {eff }}$

$$
N_{e f f}=\frac{1}{\sum_{i=1}^{N_{s}}\left(\widehat{w}_{k}^{i^{2}}\right)}
$$

where $\widehat{w}_{k}^{i}$ is the normalized weight given by

$$
\widehat{w}_{k}^{i}=\frac{w_{k}^{i}}{\sum_{i=1}^{N} w_{k}^{i}}
$$

such that $\sum \widehat{w_{k}^{l}}=1$. Resampling is then completed when $N_{e f f} \leq R_{p} N_{s}$, where $R_{p}$ is the resampling percentage, which is chosen by the user. 


\subsection{Unscented Kalman Filter}

The unscented Kalman filter utilizes the unscented transformation [31]. The UKF was developed to improve on some of the drawbacks of the EKF. The KF and EKF utilize Gaussian distributed covariances that are propagated through a linearized system, which can result in large errors due to the linearization. The UKF uses the unscented transformation to determine a minimum set of sample points, or sigma points, about the mean. These sigma points are propagated through the nonlinear system dynamics to reestimate the true mean and variance.

The UKF has been noted to be more accurate depending on the system. Typically systems with high nonlinearity benefit from utilizing the UKF over the EKF. Furthermore, the UKF does not require linearization. Depending on the complexity of the model, hydraulic systems can often have discontinuous functions within the nonlinear model. These types of systems can be difficult to implement in an EKF because the model must be split into locally continuous nonlinear functions, which are then linearized. The UKF was not tested extensively in this work, however. As will be shown in the results section, the UKF did not improve estimation over the EKF for the lower order models, likely due to the relative simplicity of the models despite being nonlinear. Formulation of the UKF is given in detail in [21].

\subsection{Observability}

Observability is a necessary analysis of filtering algorithms. If a system is observable given a set of measurements, which are functions of the states, then the all the states can be reconstructed from those measurements, given a model. Note that there are 
two types of measurements being discussed when talking about filtering and this work. There are the measurements that are taken from the hydraulic system, and there are measurements being used as the measured output equations in the filter. The observability matrix is

$$
O=\left[C C A C A^{2} \ldots C A^{n-1}\right]^{\mathrm{T}},
$$

where $n$ is the number of states. If the rank of the matrix is equal to $n$, then the system is observable, where the rank is the number of linearly independent rows or columns. Consider the linearized, first order model, with $K_{2}$ as a parameter being estimated, shown as

$$
\boldsymbol{A}_{\boldsymbol{k}}=\left[\begin{array}{cc}
1+h\left(\frac{\beta}{\mathrm{V}_{\mathrm{d}}}\left(-\frac{K_{w}\left(u_{w}^{*}+w_{0}\right)}{2 \sqrt{P_{D}^{*}-P_{L}^{*}}}-K_{1}-\frac{K_{2}^{*}}{\sqrt{P_{D}^{*}}}\right)\right) & -h \frac{\beta}{V_{d}} \sqrt{P_{D}^{*}} \\
0 & 1
\end{array}\right]
$$

The matrix would appear extremely similar if $K_{1}, K_{3}$, or $K_{4}$ was estimated. For this model, the only filter measurement is discharge pressure, so $C$ is

$$
\boldsymbol{C}=\left[\begin{array}{ll}
1 & 0
\end{array}\right]
$$

The resulting observability matrix is then

$$
\boldsymbol{O}=\left[\begin{array}{cc}
1 & 0 \\
1+h\left(\frac{\beta}{\mathrm{V}_{\mathrm{d}}}\left(-\frac{K_{w}\left(u_{w}^{*}+w_{0}\right)}{2 \sqrt{P_{D}^{*}-P_{L}^{*}}}-K_{1}-\frac{K_{2}^{*}}{\sqrt{P_{D}^{*}}}\right)\right) & -h \frac{\beta}{V_{d}} \sqrt{P_{D}^{*}}
\end{array}\right]
$$

which is full rank. The system is observable for the first order model for estimating any of the flow loss parameters individually. Consider a linearized, discrete first order model with two generalized parameters being estimated,

$$
\boldsymbol{A}_{\boldsymbol{k}}=\left[\begin{array}{ccc}
a_{11} & a_{12} & a_{13} \\
0 & 1 & 0 \\
0 & 0 & 1
\end{array}\right]
$$


and only the first state measured

$$
\boldsymbol{C}=\left[\begin{array}{lll}
1 & 0 & 0
\end{array}\right]
$$

The observability matrix is then

$$
\boldsymbol{O}=\left[\begin{array}{ccc}
1 & 0 & 0 \\
a_{11} & a_{12} & a_{13} \\
a_{11} a_{11} & a_{11} a_{12} & a_{11} a_{13}
\end{array}\right]
$$

which is clearly not full rank and therefore not observable. Therefore, estimating any two parameters in a first order model, where the only filter measurement is the first state, is not feasible. This result is somewhat obvious when considering linearizing the flow loss model with respect to discharge pressure,

$$
\frac{\partial Q_{L}}{\partial P_{D}}=K_{1}+\frac{K_{2}}{\sqrt{P_{D}^{*}}}=K_{1}+K_{2}^{\prime}
$$

There is nothing unique to separate $K_{1}$ from $K_{2}{ }^{\prime}$, therefore estimating both at the same time is not practical.

A similar analysis was done for the second order model and eighth order model. The table below shows combinations of filter measurements and observability analysis, where $p$ denotes the number of parameters estimated. Note that if a system is observable with a set of measurements, then adding a measurement will not change its observability. The term "filter" indicates the measurement was utilized as a filter measurement, and "input" indicates the measurement was only utilized as an input to the model. 
Table 1. Observability analysis.

\begin{tabular}{lllllllll}
\hline \hline Model & $P_{D}$ & $P_{L}$ & $P_{C}$ & $\alpha$ & $u_{w}$ & $L_{L}$ & $p$ & Obs? \\
\hline 1 & Filter & Input & --- & Input & Input & --- & 1 & Yes \\
1 & Filter & --- & --- & Input & --- & Input & 1 & Yes \\
1 & Filter & Input & --- & Input & Input & --- & 2 & No \\
2 & Filter & Input & --- & --- & Input & --- & 1 & Yes $^{*}$ \\
2 & Filter & Input & --- & Filter & Input & --- & 1 & Yes \\
2 & Filter & --- & --- & Filter & --- & Input & 1 & Yes \\
2 & Filter & Input & --- & Filter & Input & --- & 2 & No \\
2 & Filter & Input & Filter & --- & Input & --- & 1 & Yes \\
8 & Filter & Filter & --- & --- & Input & --- & 1 & Yes \\
8 & Filter & Filter & --- & --- & Input & --- & 2 & No* \\
\hline \hline
\end{tabular}

Two of the observability results are somewhat misleading and are indicated with a * in the observability column. For the second order model, the system was found to be observable without utilizing information about the swash plate as an input or filter measurement. However, implementing this setup for the filter resulted in extremely inaccurate estimation that increased over time. Recall the discharge pressure equation,

$$
\dot{P}_{D}=\frac{\beta}{V_{d}}\left(V_{p} \omega_{m} \frac{\alpha}{\alpha_{\max }}-K_{w}\left(u_{w}+w_{0}\right) \sqrt{P_{D}-P_{L}}-\left(K_{1} P_{D}+K_{2} \sqrt{P_{D}}\right)\right)
$$

For a given test where $\alpha$ is unknown and $K_{2}$ is the sole parameter being estimated, $\alpha$ and $K_{2}$ would have some correct value that would result in the correct discharge pressure dynamics. The problem is that there is a set of infinite combinations of $\alpha$ and $K_{2}$ that could result in the same discharge pressure dynamics. This result is obvious in the steady state, but is also true dynamically.

Although the $8^{\text {th }}$ order model was shown to be unobservable, simulation data showed convergence. The converge to the correct $K_{1}$ and $K_{2}$ in simulation data, but only for a perpetually excited system - this is discussed in greater detail in the results. One of the major drawbacks of the $8^{\text {th }}$ order model however, is the step size required for the model 
is much smaller than what could be considered a reasonable sampling rate of the measurements, and so this phenomenon was not investigated in depth due to the impracticality of the high order model. 


\section{CHAPTER 4: SIMULATION RESULTS AND DISCUSSION}

\subsection{Eighth Order Model State Estimation}

Measurement data for the high order model was simulated for several seconds with a constant time increment of $0.2 \mathrm{~ms}$. The input was a square function, switching between $1 \pm 0.14$ every second to create transient responses. The input has been modeled as the flow control valve opening, which is similar to how an operator would control the flow to a hydraulic load to control velocity. The states, measurements, and inputs are

$$
x=\left[\begin{array}{c}
P_{D} \\
P_{C} \\
P_{L} \\
y_{v} \\
\dot{y}_{v} \\
y_{b} \\
\dot{y}_{b} \\
\omega
\end{array}\right], \quad z=\left[\begin{array}{c}
P_{D} \\
P_{L}
\end{array}\right], \text { and } u=u_{v} \text {. }
$$

The process and measurement noise can be chosen somewhat arbitrarily, since that is the noise used both in the simulation and filter. Values were chosen that were smaller than the magnitudes of the range of each state. The process and measurement noise vector used in model simulations is given by

$$
v(t)=\left[\begin{array}{l}
1000 \\
1000 \\
1000 \\
1 e^{-7} \\
1 e^{-3} \\
1 e^{-7} \\
1 e^{-3} \\
1 e^{-3}
\end{array}\right] \text { and } n(t)=\left[\begin{array}{l}
10000 \\
10000
\end{array}\right]
$$

The simulated results of the estimation of both filters, as well as the calculated swash plate angle and volumetric efficiency, are: 


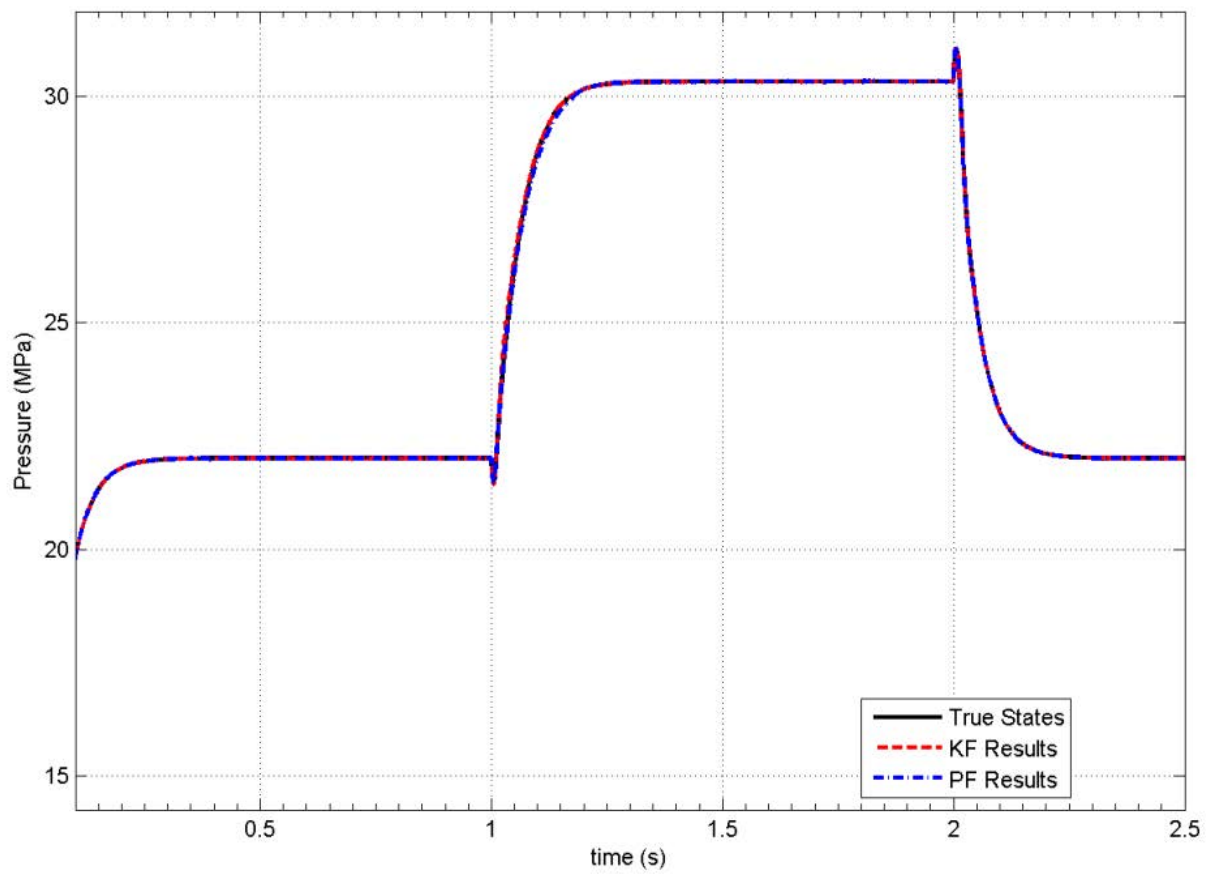

Figure 11. Comparison of Kalman and Particle filter for estimating discharge pressure.

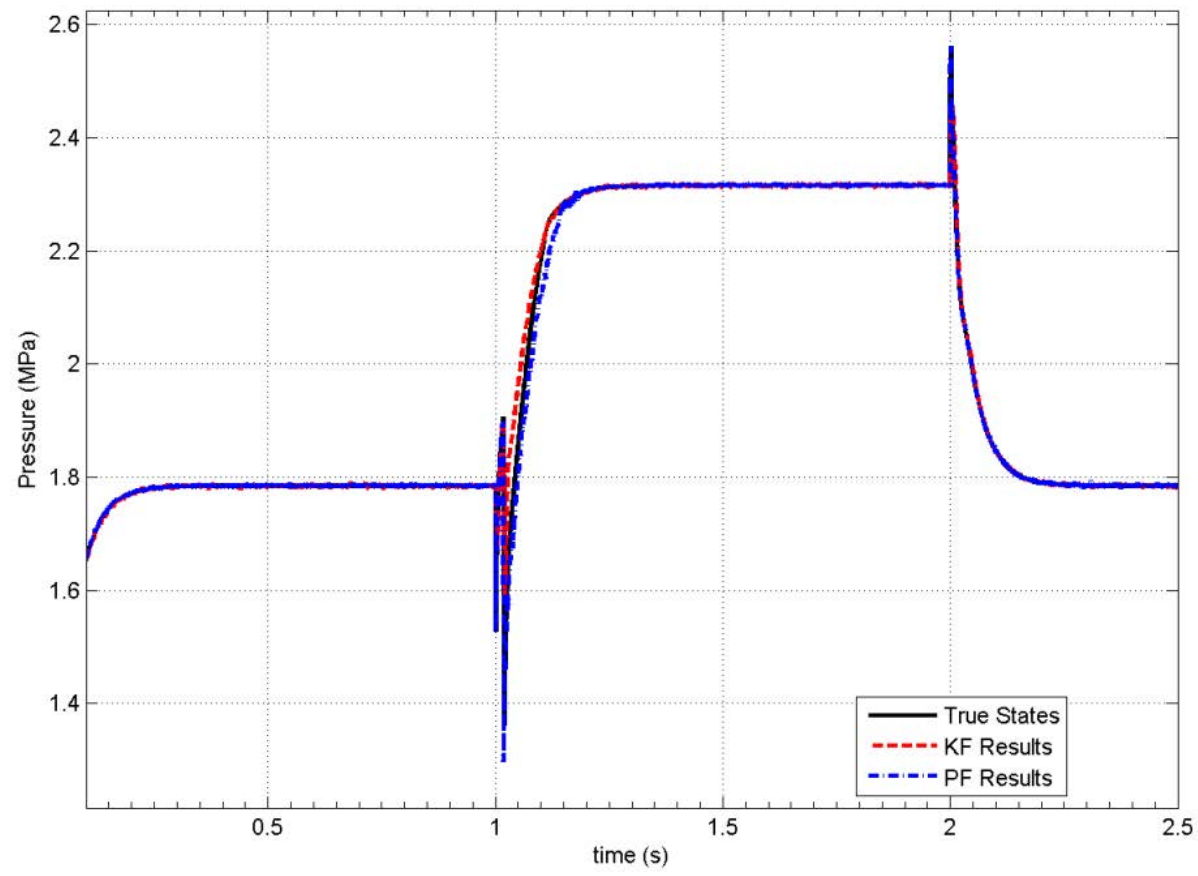

Figure 12. Comparison of Kalman and Particle filter for estimating control pressure. 


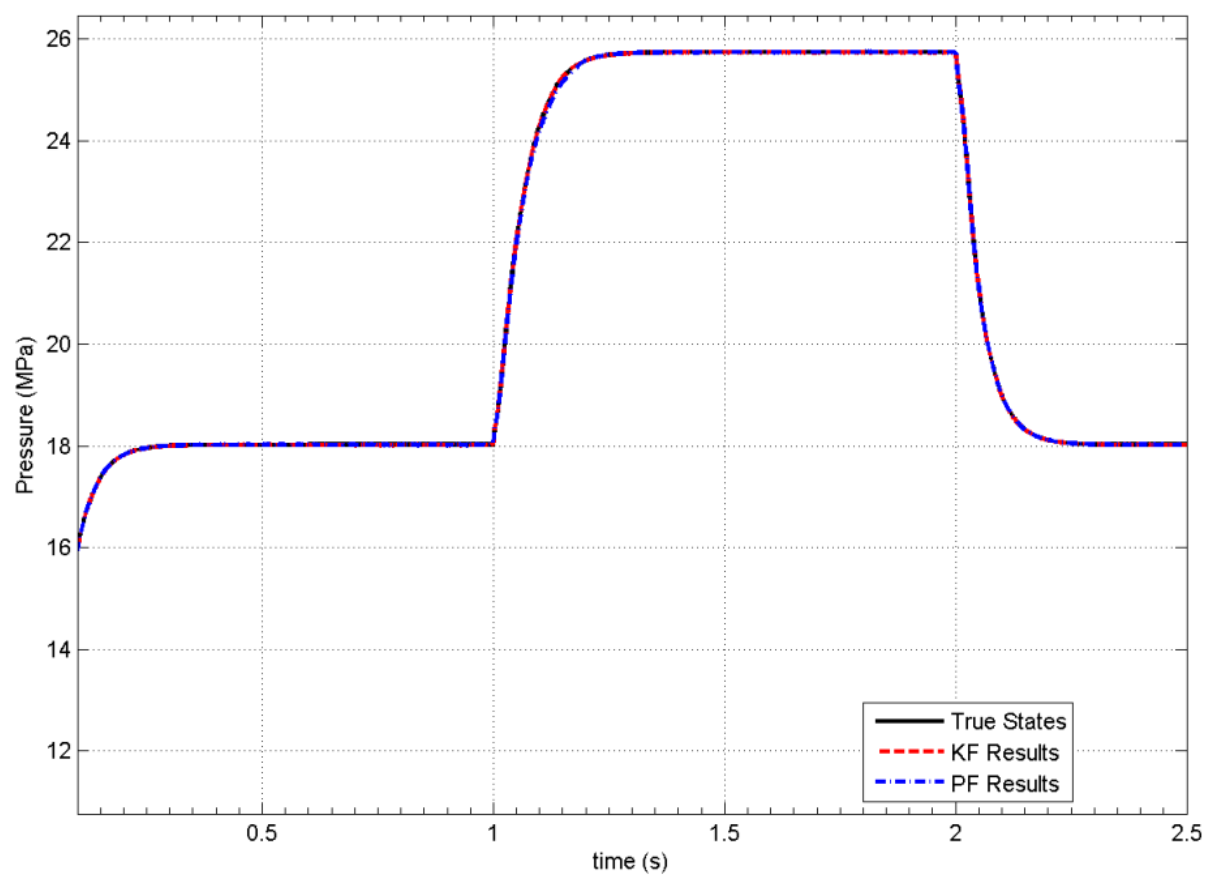

Figure 13. Comparison of Kalman and Particle filter for estimating load pressure.

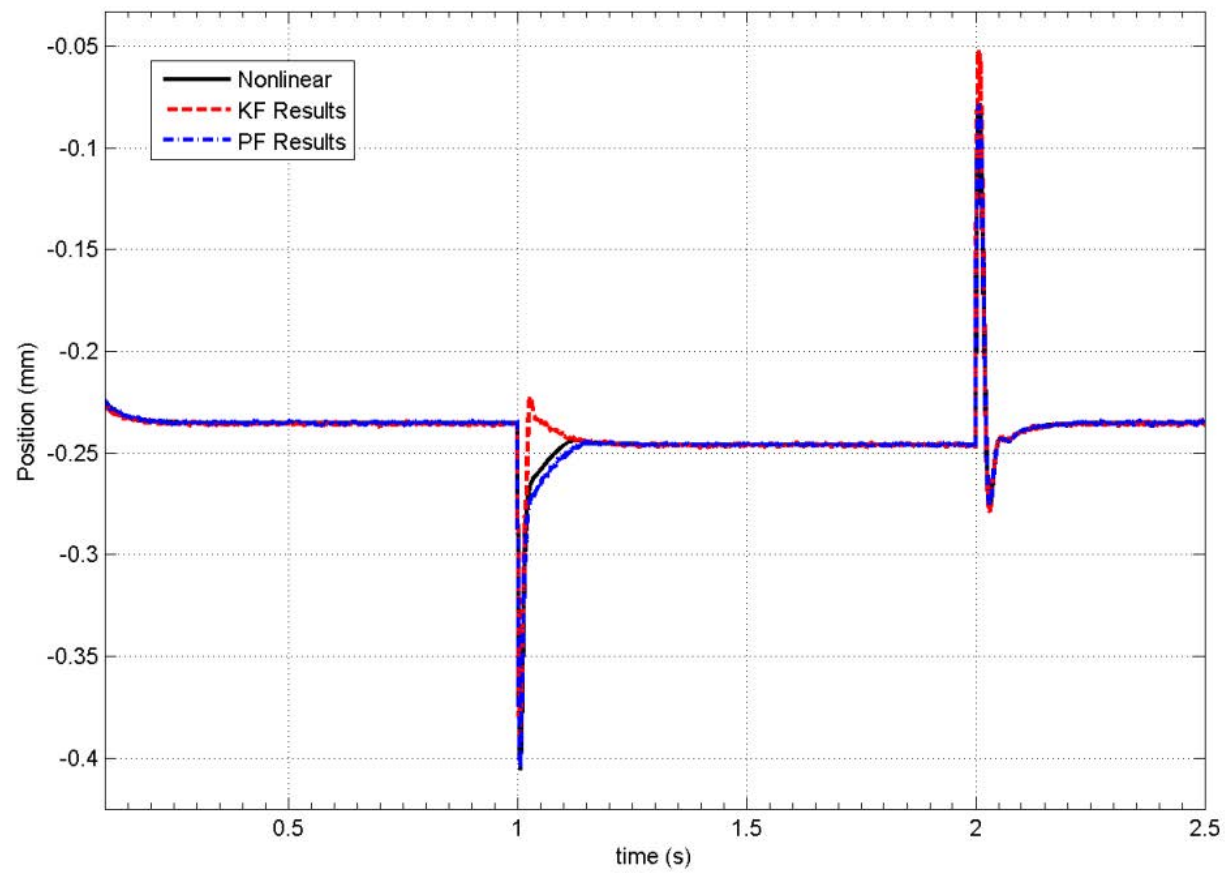

Figure 14. Comparison of Kalman and Particle filter for estimating valve position. 


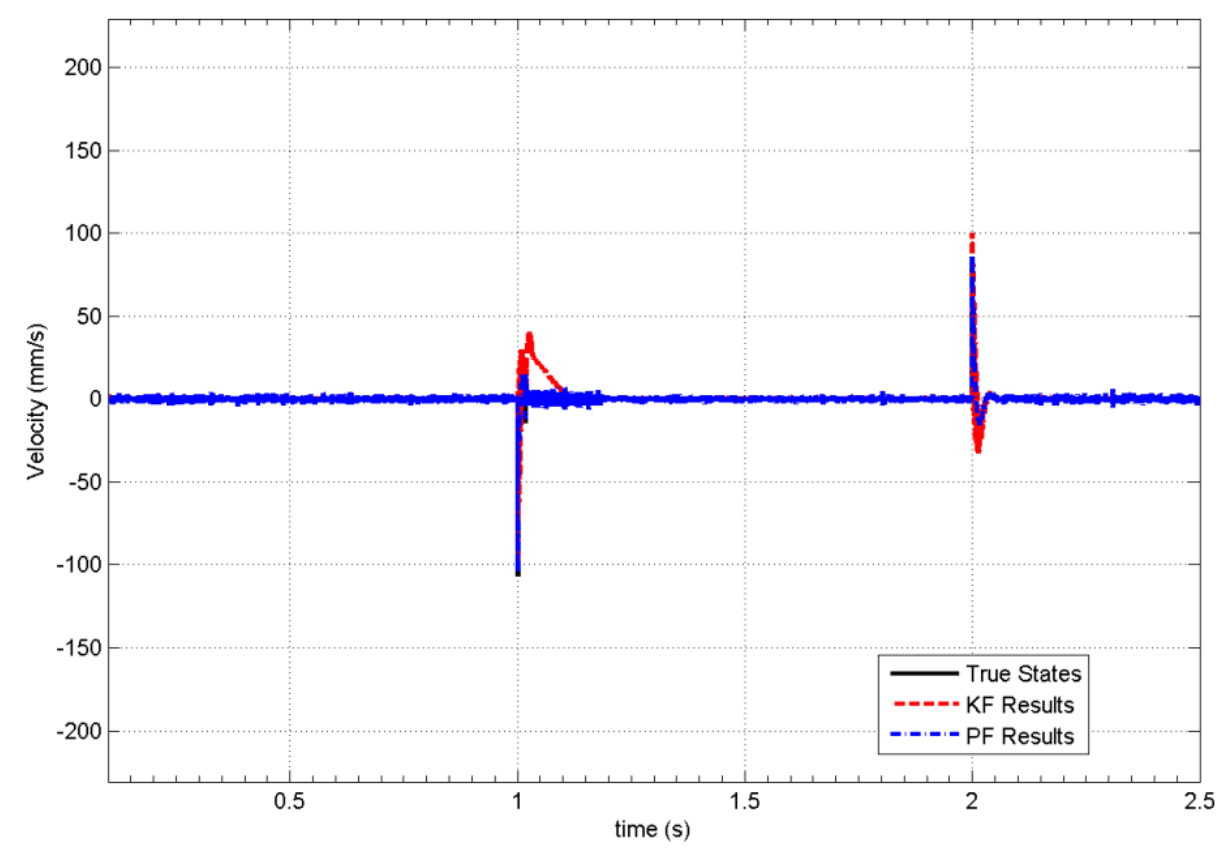

Figure 15. Comparison of Kalman and Particle filter for estimating valve velocity.

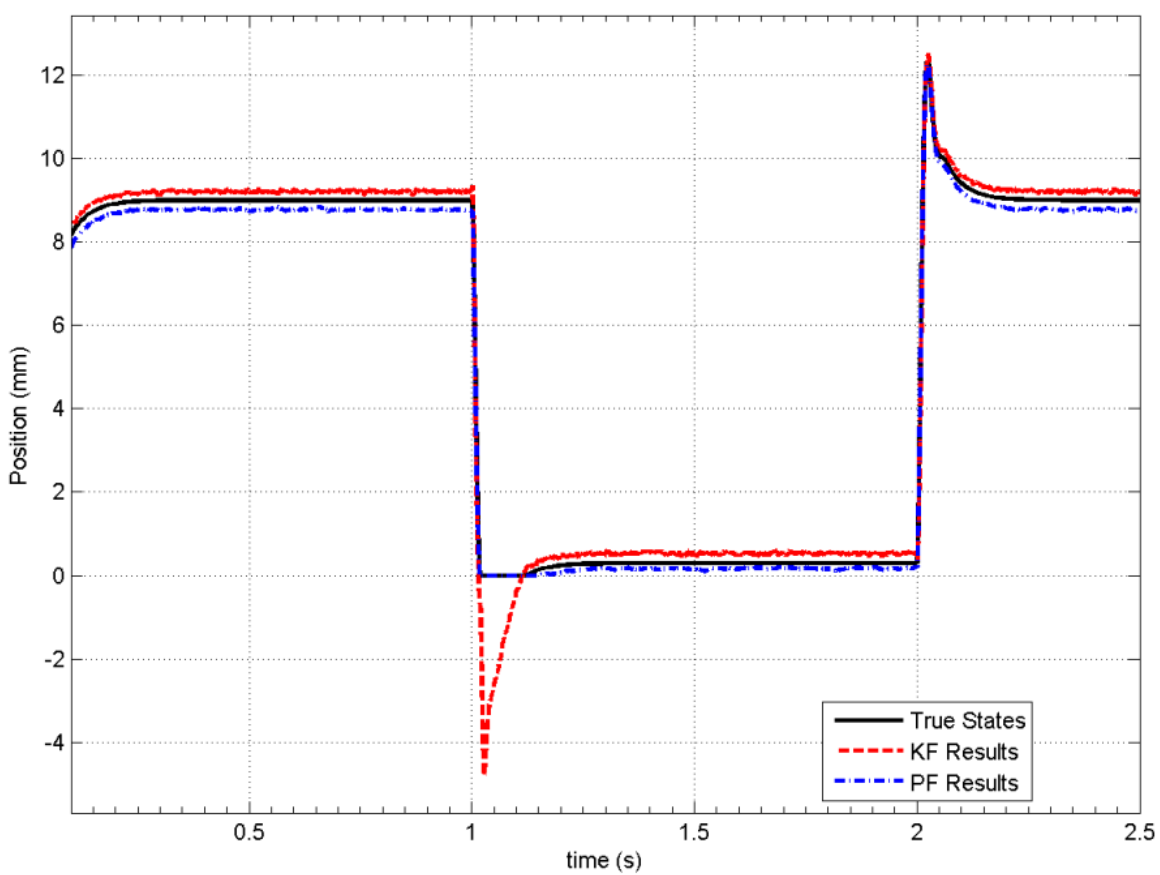

Figure 16. Comparison of Kalman and Particle filter for estimating actuator position. 


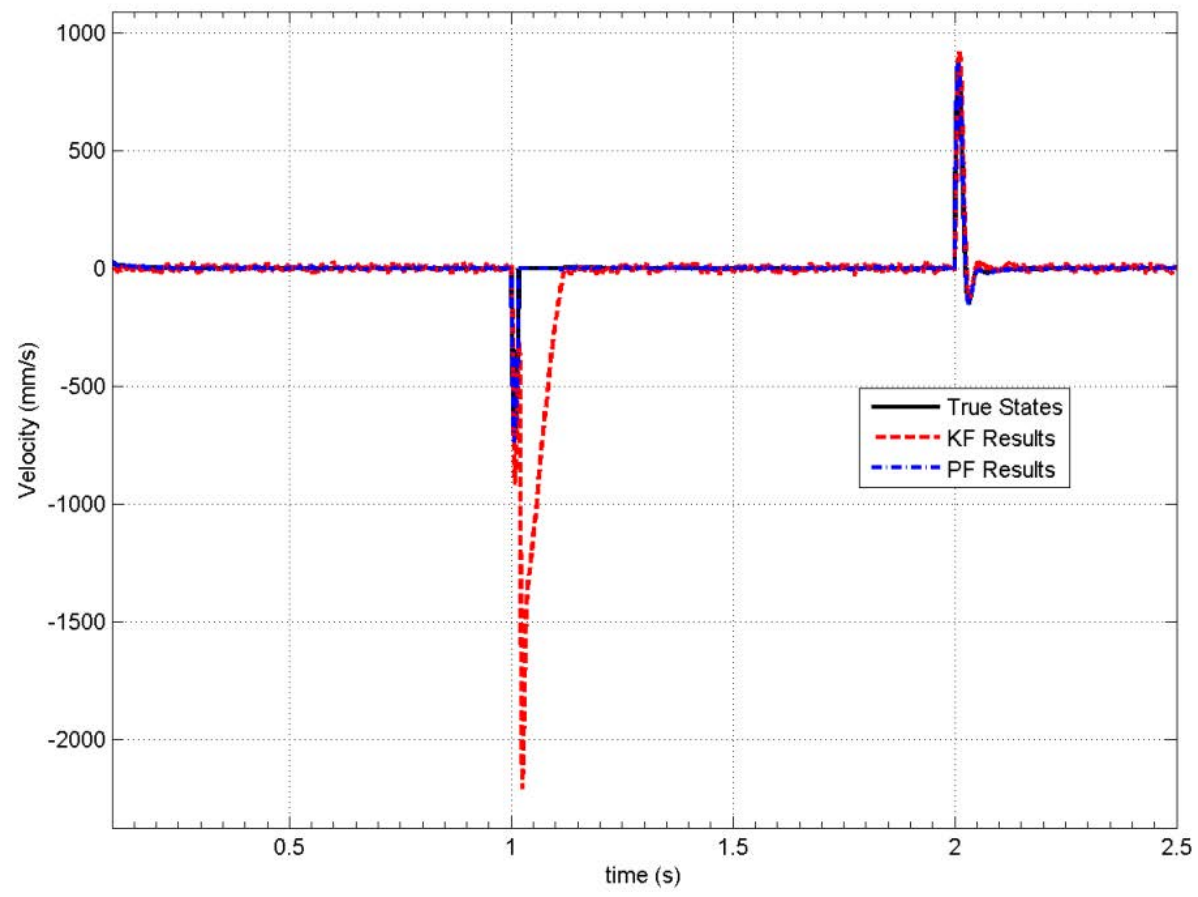

Figure 17. Comparison of Kalman and Particle filter for estimating actuator velocity.

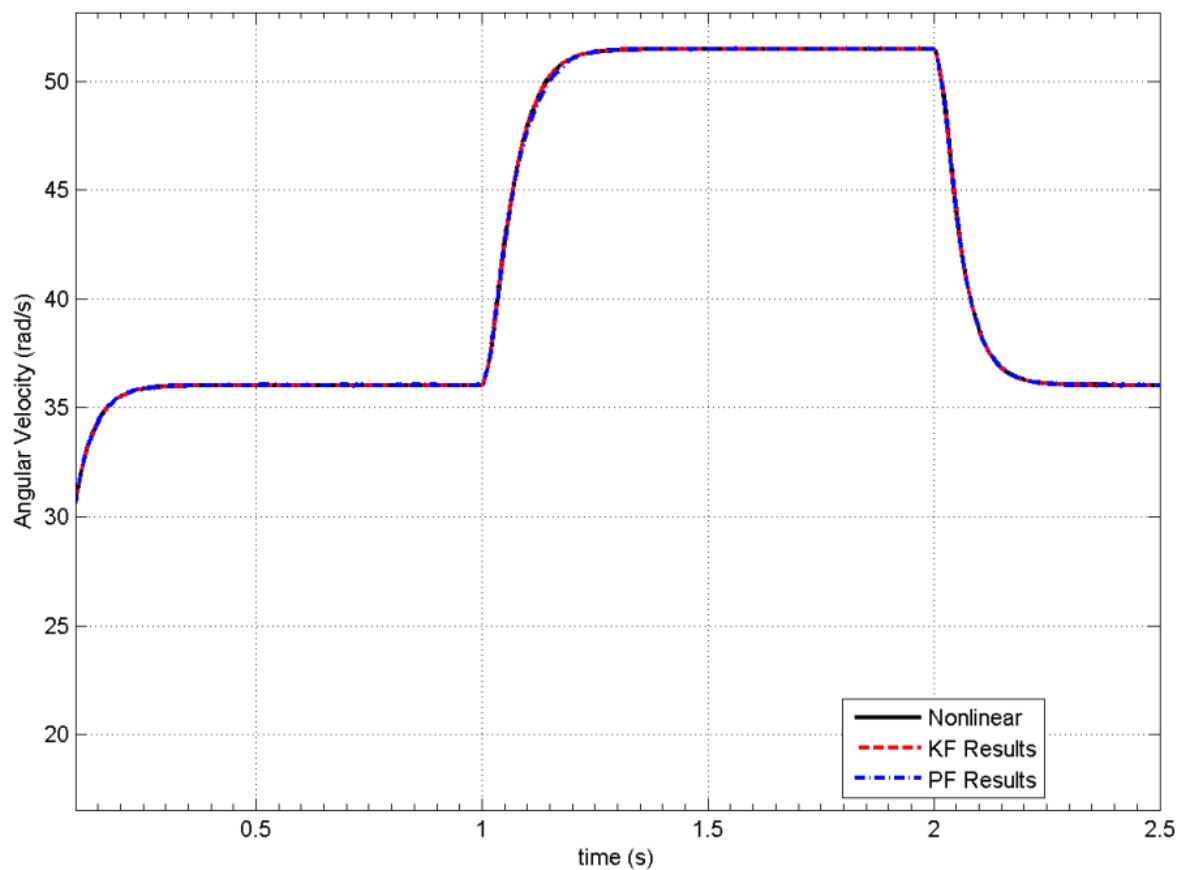

Figure 18. Comparison of Kalman and Particle filter for estimating motor load velocity. 
Estimation of the three pressures are extremely similar for both filters, as is shown in Figs. 11-13. Motor load velocity is also estimated extremely well for both filters. Position and velocity of the valve and actuator have regions of good estimation, but not over the entire simulation. Specifically, from approximately $1 \mathrm{~s}$ to $1.1 \mathrm{~s}$ there is a noticeable difference between the two filters. This is likely due to the actuator position. During this period, the limit of its position (saturation at $0 \mathrm{~m}$ ) has been reached. The linearized model for the KF does not consider saturation. However, the PF, since it utilizes a nonlinear model in the filter, can have the saturation within the model and filter calculation. As Fig. 16 shows, the KF continues pass the saturation point while the PF stays at saturation. While this does show some overall benefit to the PF, states can be limited in the KF algorithm. The KF algorithm also recovers, despite going into an unrealistic estimation briefly. The $\mathrm{PF}$ is also a much slower and more complex algorithm. The benefits of the PF were not substantial enough to continue utilizing for the rest of the results.

\subsection{Eighth Order Model Parameter Estimation}

Simulations of the high order modeling equations with the KF and EKF filters in various configurations were used to demonstrate the parameter and efficiency capabilities. Under specific conditions, the KF and EKF estimation are practically the same. One such condition is for estimating a single parameter. The states, measurements, and inputs are 


$$
x=\left[\begin{array}{c}
P_{D} \\
P_{C} \\
P_{L} \\
y_{v} \\
\dot{y}_{v} \\
y_{b} \\
\dot{y}_{b} \\
\omega \\
K_{1} \\
K_{2}
\end{array}\right], \quad z=\left[\begin{array}{l}
P_{D} \\
P_{L}
\end{array}\right], \text { and } u=u_{v}
$$

Note that for simulations where only 1 parameter was estimated, say $K_{2}$, then there were only 9 states. Therefore, $K_{1}$ was no longer a state and the corresponding process noise row was omitted as well. The process and measurement noise vector used in model simulations is given by

$$
v(t)=\left[\begin{array}{c}
10 \\
10 \\
10 \\
1 e^{-9} \\
3 e^{-5} \\
3 e^{-5} \\
1 e^{-6} \\
1 e^{-2} \\
1 \\
1
\end{array}\right] \text { and } n(t)=\left[\begin{array}{c}
1 e^{3} \\
1 e^{3}
\end{array}\right]
$$

In Figs. 19-28, the FCV valve was kept constant during the simulation. The only parameter included for estimation was $K_{1}\left(K_{2}\right.$ set to zero), which has been normalized to 1 . At 2.5 seconds, $K_{1}$ was increased by $50 \%$. The only noticeable difference in performance is that during the $50 \%$ increase, the KF has extremely poor estimation, but then converges back to similar estimation as the EKF in less than 0.1 seconds. 


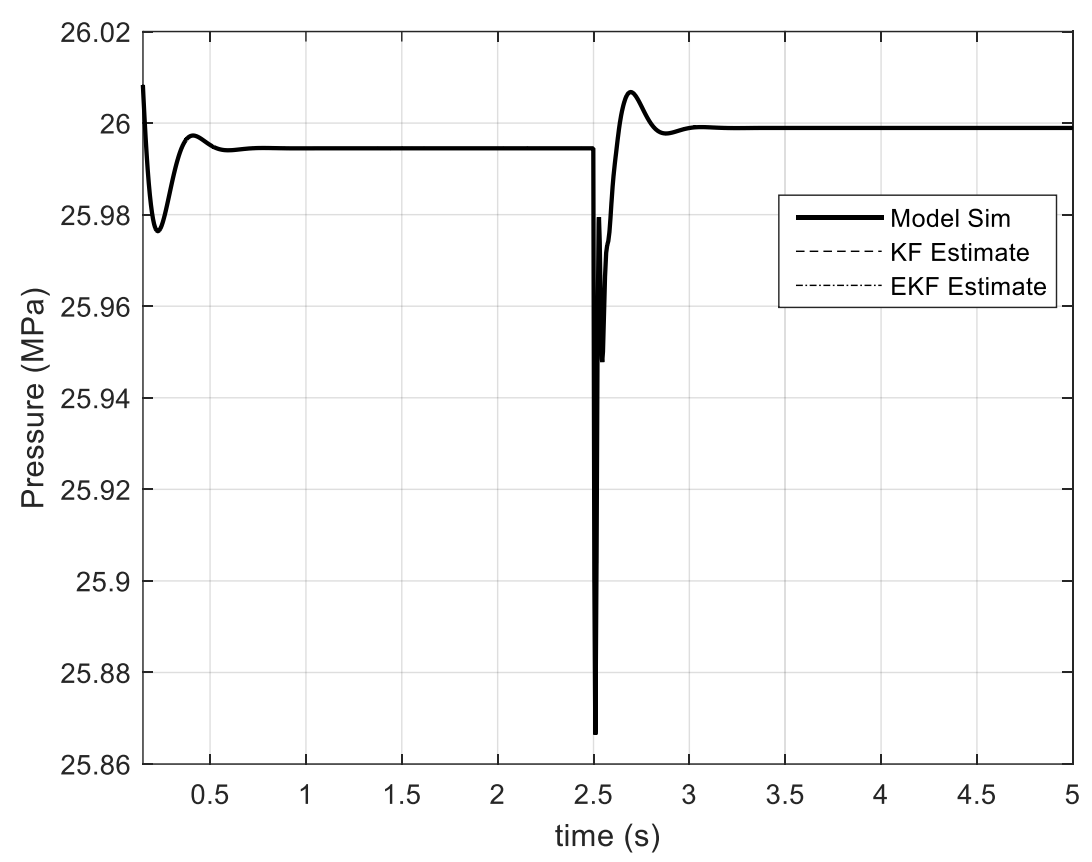

Figure 19. Discharge Pressure estimation for simulation data.

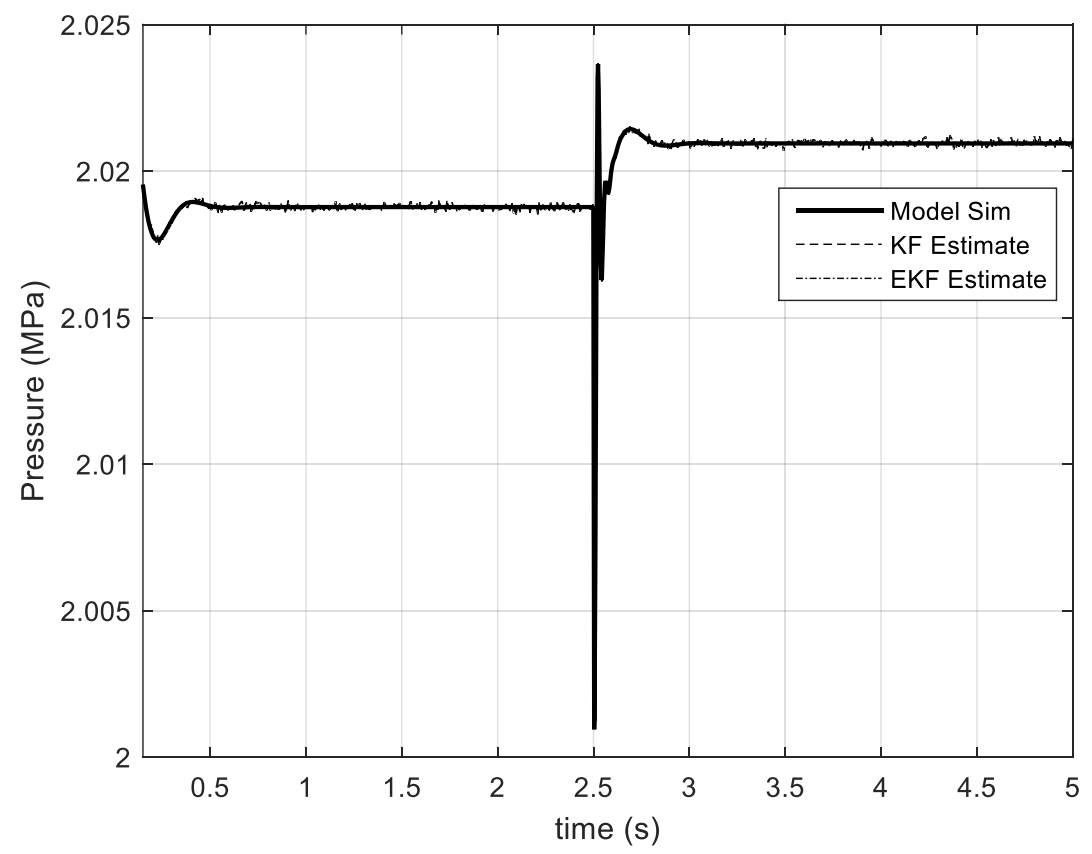

Figure 20. Control Pressure estimation for simulation data. 


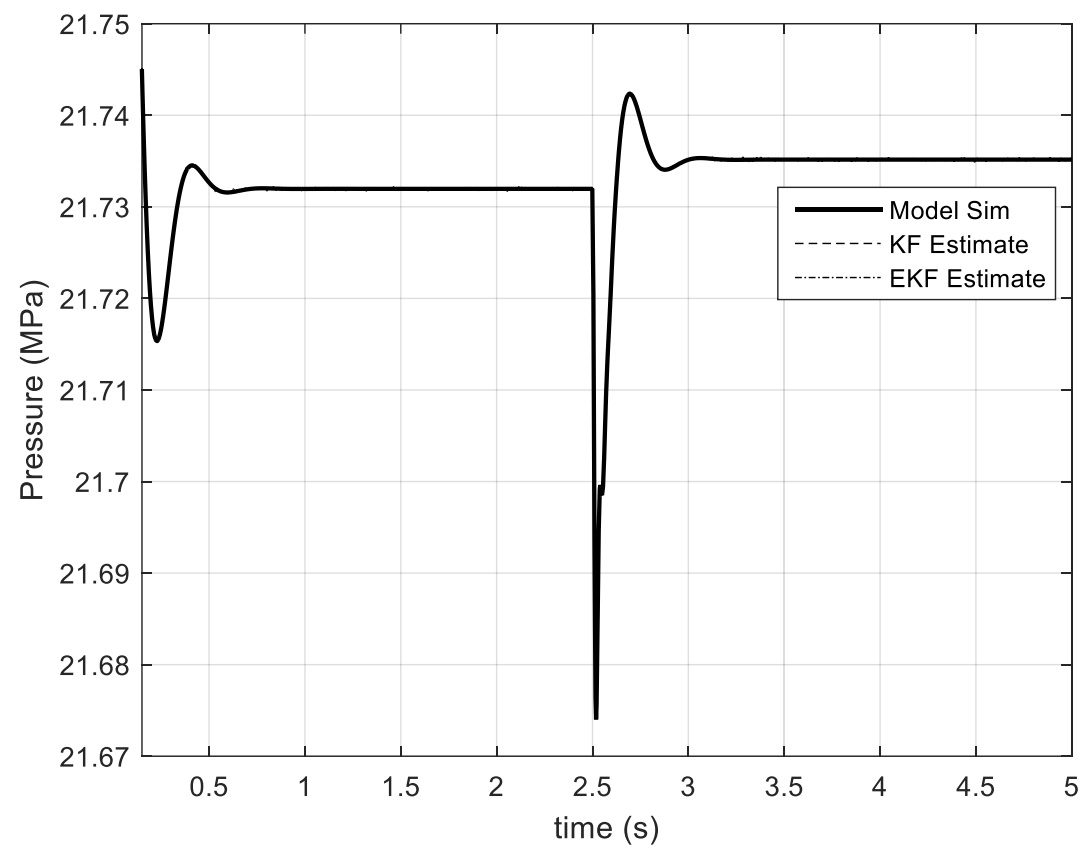

Figure 21. Load Pressure estimation for simulation data.

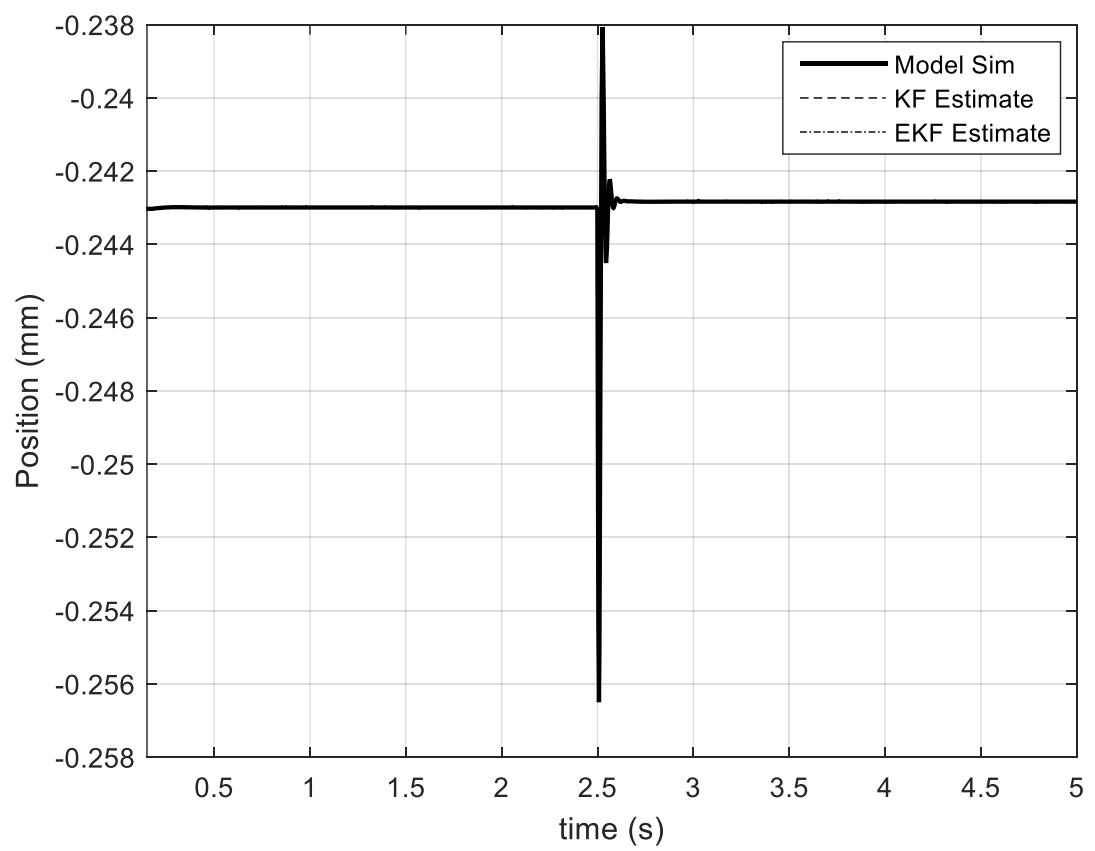

Figure 22. Control actuator valve position estimation for simulation data. 


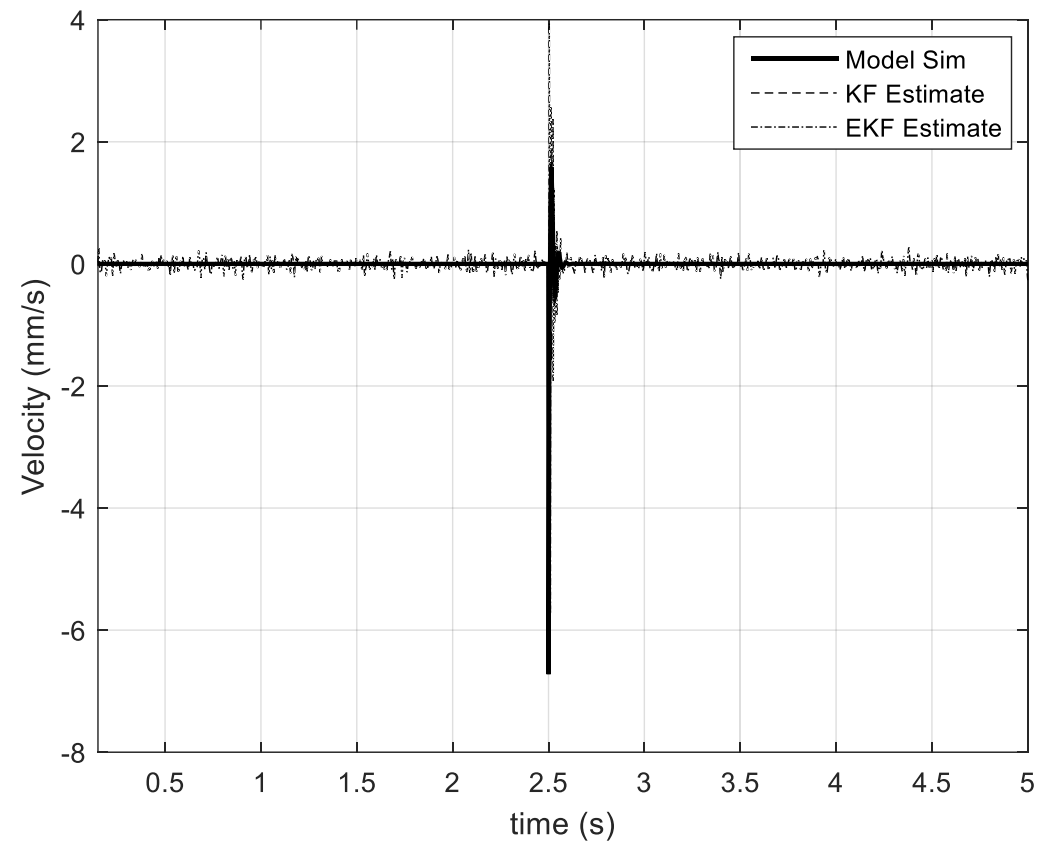

Figure 23. Control actuator valve velocity estimation for simulation data.

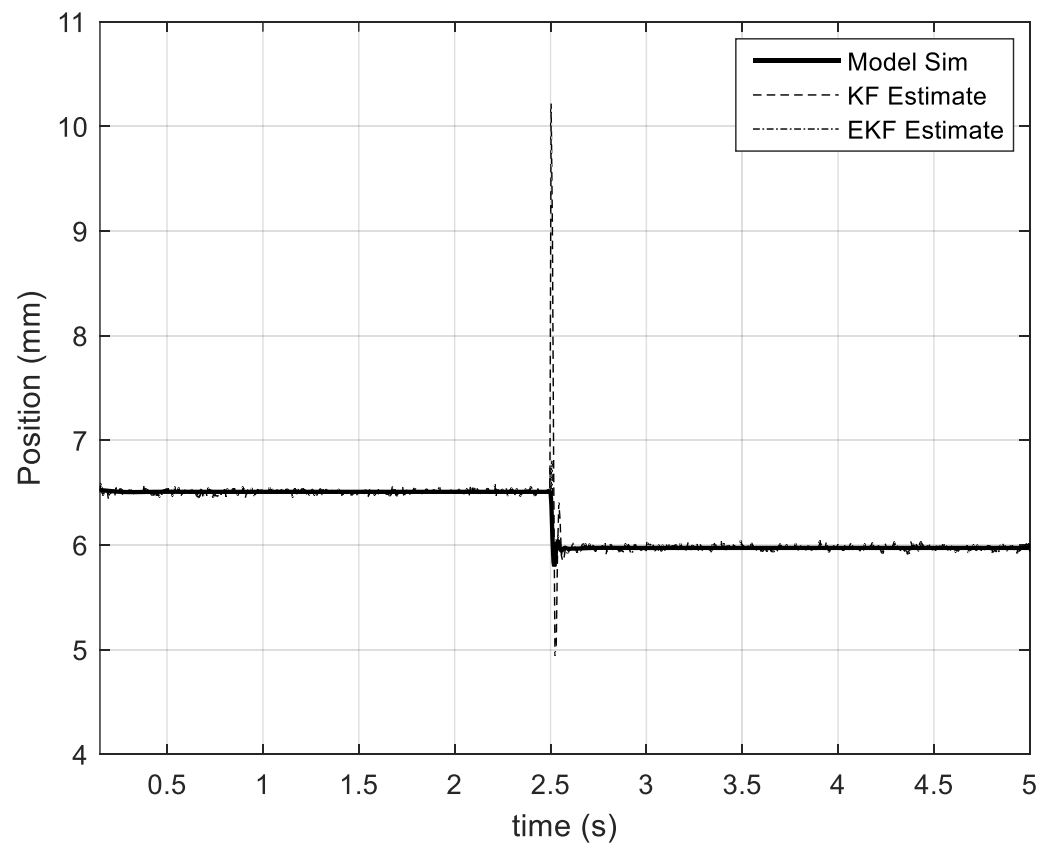

Figure 24. Piston position estimation for simulation data. 


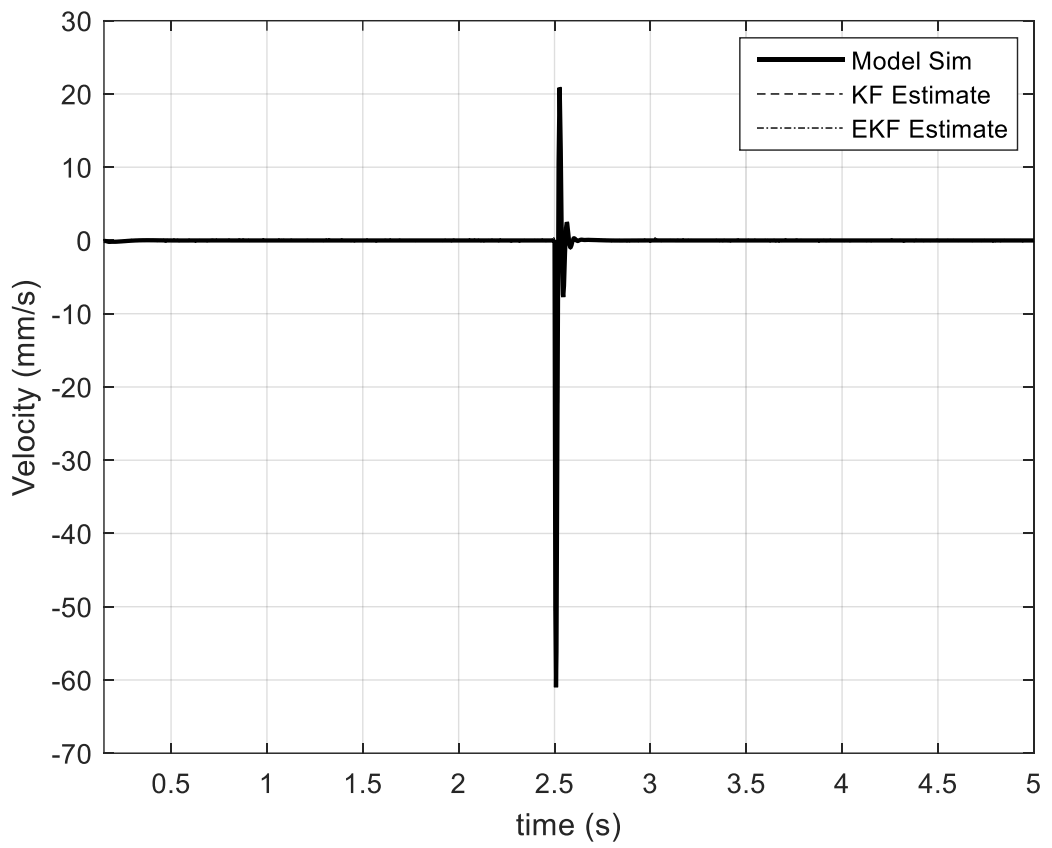

Figure 25. Piston velocity estimation for simulation data.

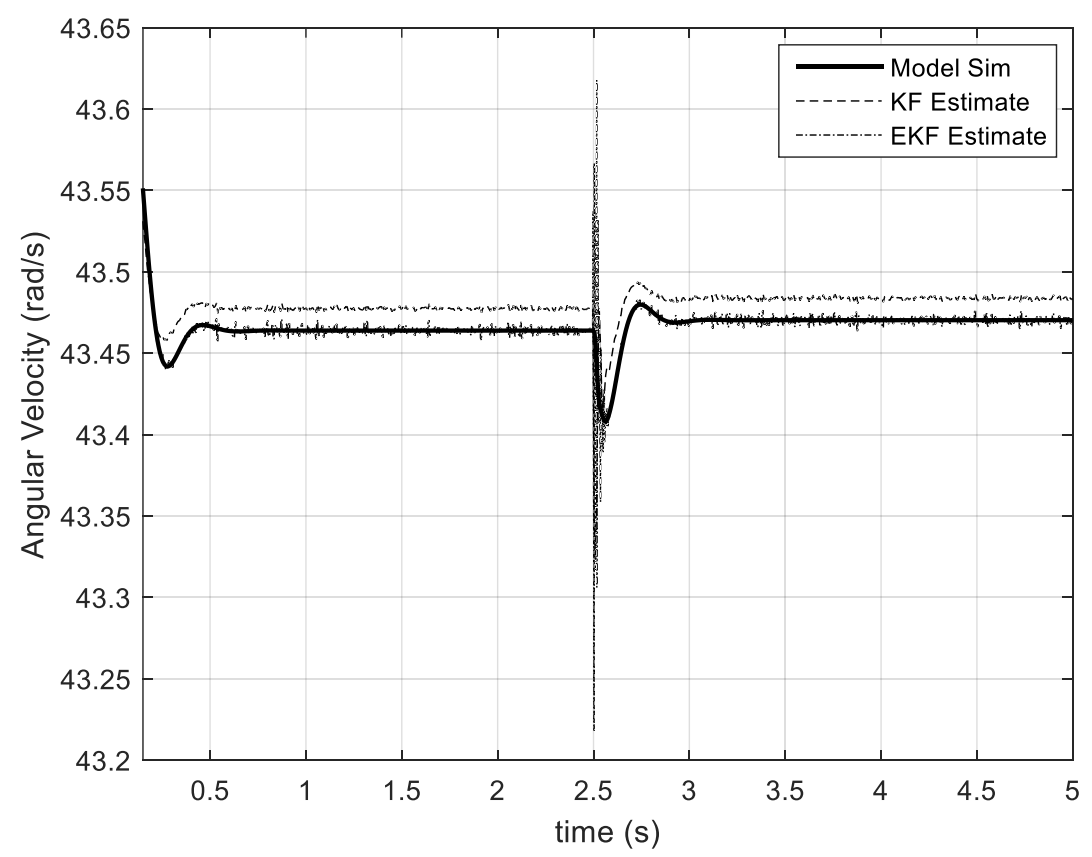

Figure 26. Output motor velocity estimation for simulation data. 


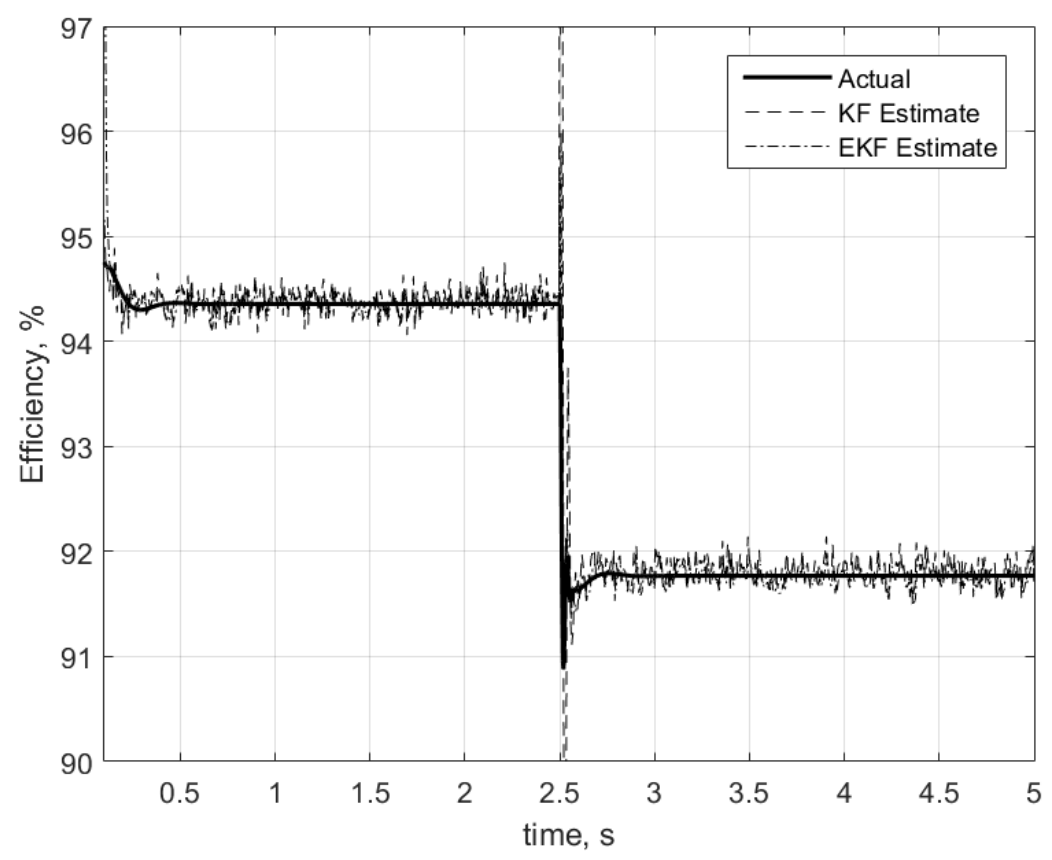

Figure 27. Volumetric efficiency estimation for a $50 \%$ step to $K_{1}$.

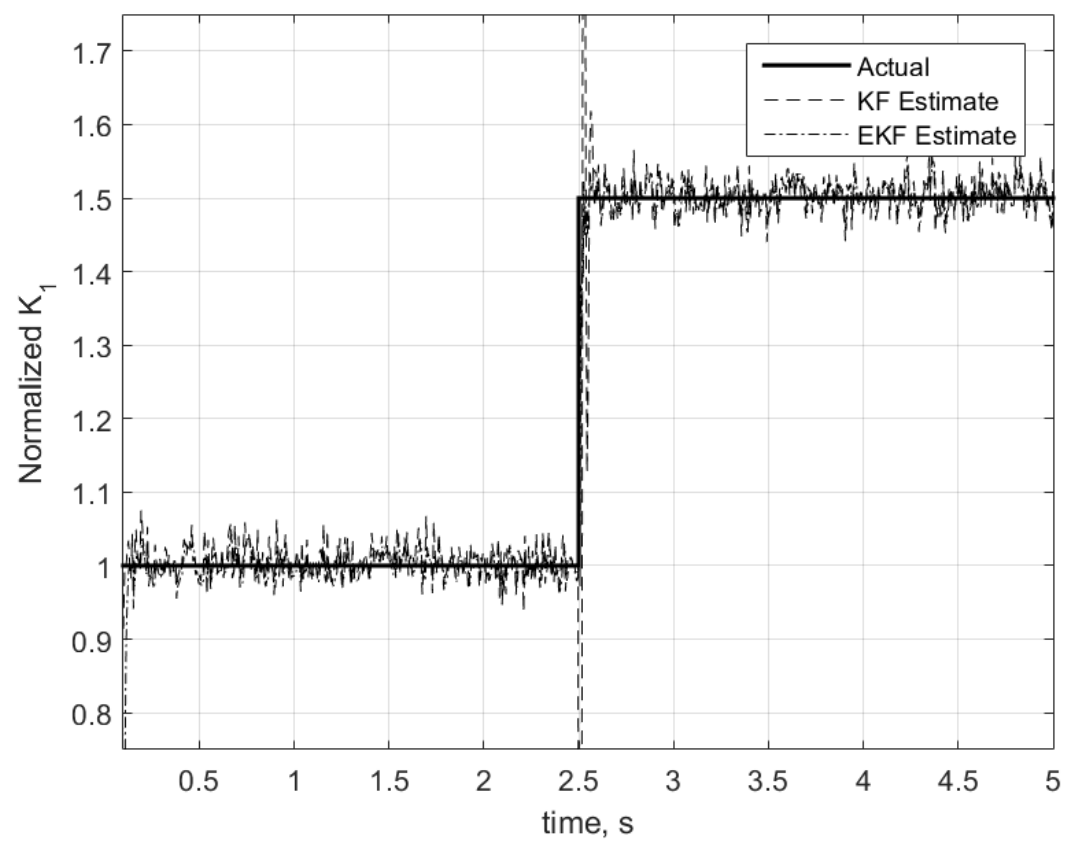

Figure 28. $K_{1}$ estimation for a $50 \%$ step to $K_{1}$. 
Though the KF can estimate $K_{1}$ or $K_{2}$ individually, the filter is not able to estimate both $K_{1}$ and $K_{2}$ at the same time. In other simulations, it was found that the estimation diverges when estimating these two parameters at the same time. The KF was expected to not be stable since observability analysis showed that the linearized augmented state equations were not observable for the case of two or more estimated parameters. However, simulations showed that the EKF can estimate both flow loss parameters simultaneously. The following simulation includes a sinusoidal input to the FCV with an amplitude $\pm 15 \%$ from the nominal valve area opening. Both $K_{1}$ and $K_{2}$ were kept constant at the normalized value of 1 for 5 seconds, and then both were increased by 50\%. Figures 29-31 shows the result of this simulation.

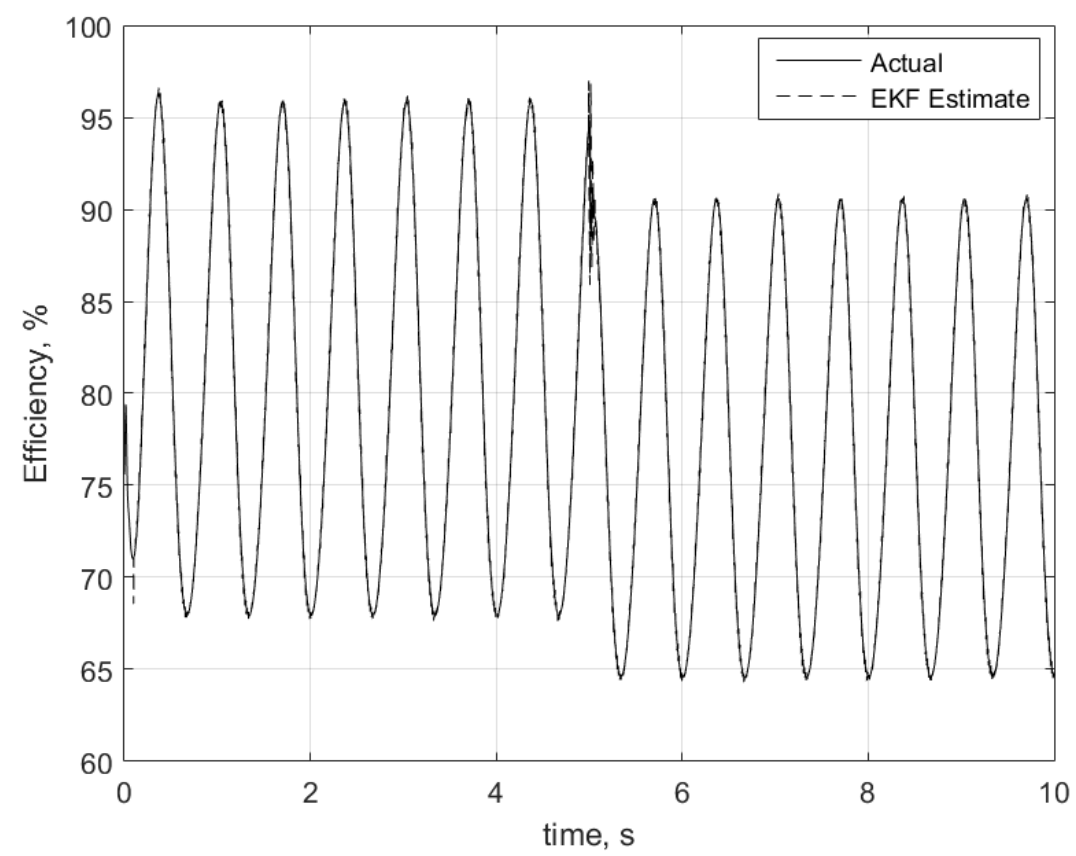

Figure 29. Volumetric efficiency estimation for a $50 \%$ step to $K_{1}$ and $K_{2}$. 


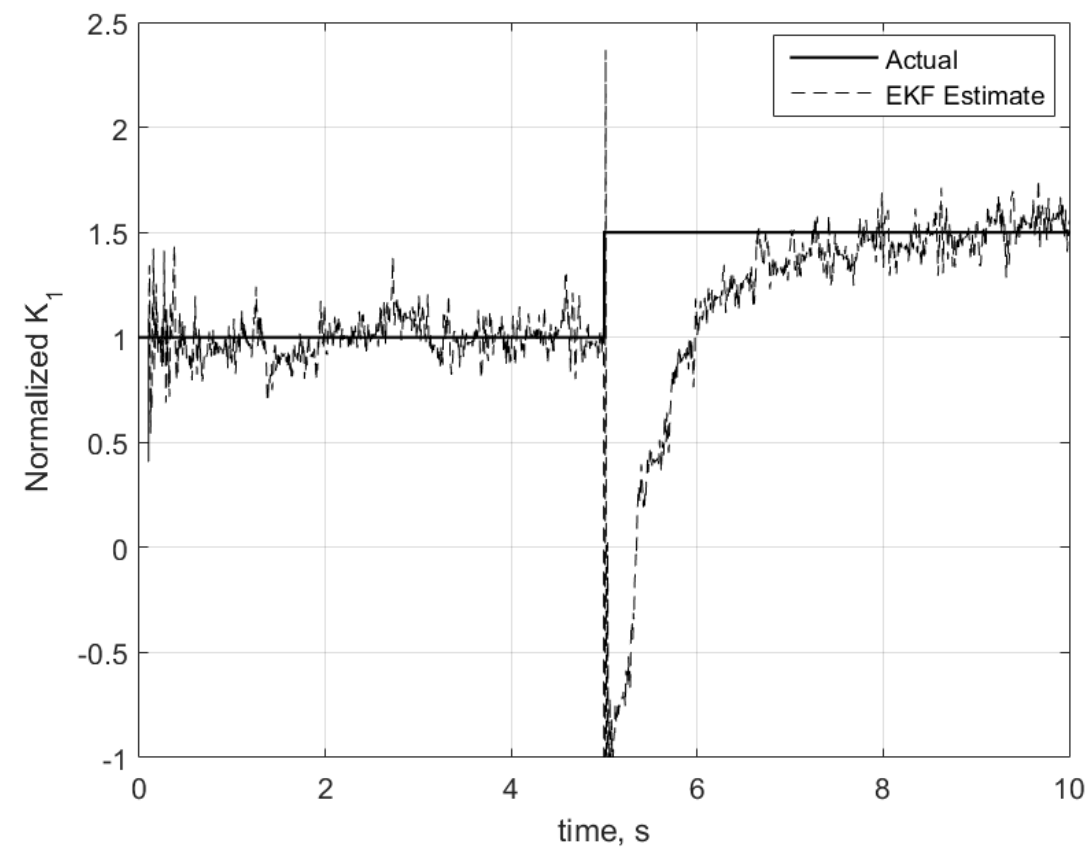

Figure 30. $K_{1}$ estimation for a $50 \%$ step to $K_{1}$ and $K_{2}$.

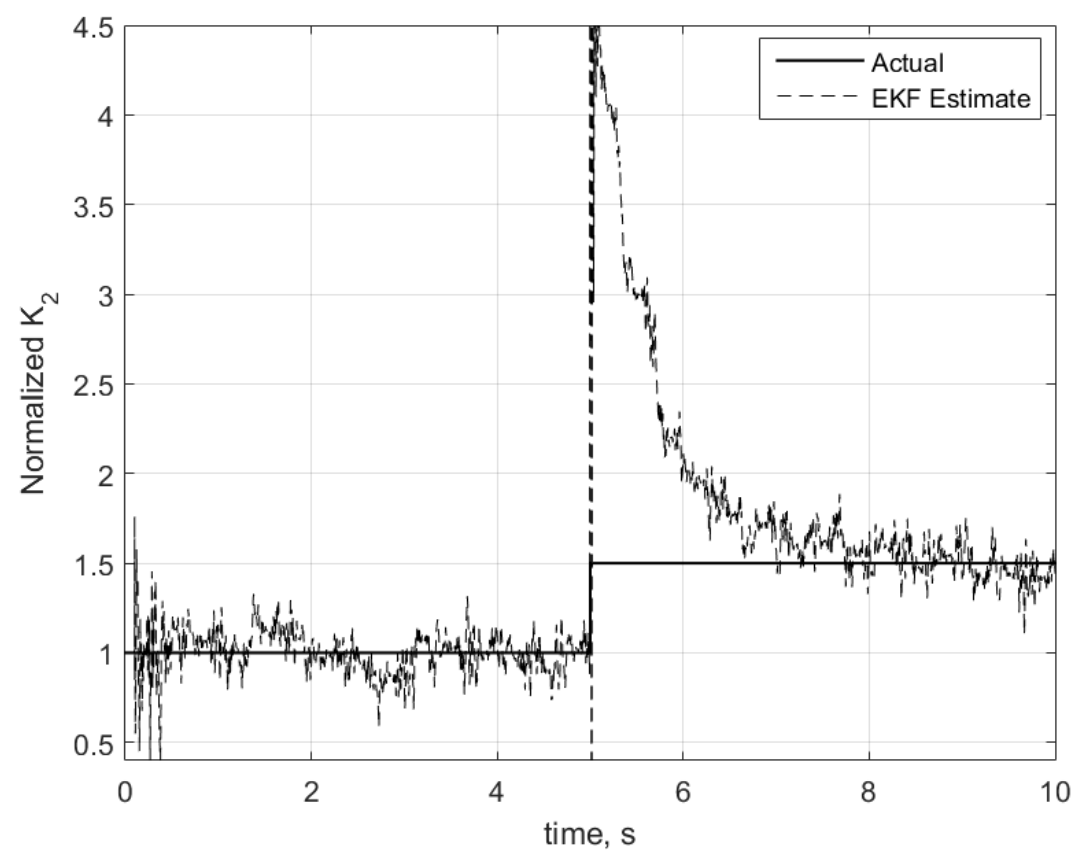

Figure $31 . K_{2}$ estimation for a $50 \%$ step to $K_{1}$ and $K_{2}$. 
The volumetric efficiency is estimated extremely well. It is nearly indistinguishable from the actual volumetric efficiency. Both $K_{1}$ and $K_{2}$ also have slowly responding but acceptable estimation. At the time of the step increase in these parameters, there is a large increase in estimation error for approximately 3 seconds before returning to acceptable estimation. The reason for the volumetric efficiency being estimated well despite a temporary poor estimation in the parameters is due to how the flow losses have been introduced into the model. The discharge pressure differential equation includes a summation of flows and time rate of change of volume. The flow losses have been included in this summation as where $Q_{L}$ is the total flow loss. It is clear

$$
Q_{L}=K_{1} P_{D}+K_{2} \sqrt{P_{D}}
$$

from Eq. (95) for a given $P_{D}$ and $Q_{L}$, there is an infinite number of solutions for $K_{1}$ and $K_{2}$. The parameter values estimated in Figs. 30 and 31 from 5 seconds to about 8 seconds (i.e. while the estimates are still converging) are perhaps $a$ solution for the flow loss Eq. (95) that was actually implemented - just not the values that were specifically chosen and used in the model. This problem is the reason for supplying a dynamic sinusoidal input to the FCV when estimating both flow loss coefficients. Varying the FCV area causes the discharge pressure and other states to vary over a range, which presumably helps the EKF to converge to the appropriate parameter estimates, which approach the true values of the parameters. Both $K_{1}$ and $K_{2}$ affect the eigenvalues of the linear model, which is a possible explanation for why continually exciting the model improves the estimation. One difference that might explain why the full order model can estimate both coefficients, but the low order model cannot, is that two of the eigenvalues of the linear model change with a different nominal value of $K_{1}$ and $K_{2}$ when the nonlinear model is linearized. Compared 
to the first order model, which only has 1 eigenvalue (ignoring the eigenvalues of 0 for the augmented coefficient states), some dynamics may be lost in the first order model that is necessary for predicting both coefficients. This phenomenon was not investigated fully, however. While the high order model shows promise, there is no practical application for it. The sample time necessary to run this model dynamically often reaches $1 \mu$ s. This small step size is simply not feasible in any industry setting. The model also does not run in real time. Furthermore, sensor measurements are not going to be taken at small of a step time either, which makes the model difficult to implement in a filter as well. The rest of the work, including the experimental results, focuses solely on the first and second order model due to these constraints of the eighth order model. 


\section{CHAPTER 5: EXPERIMENTAL SETUP}

The experimental setup is shown in Fig. 32.

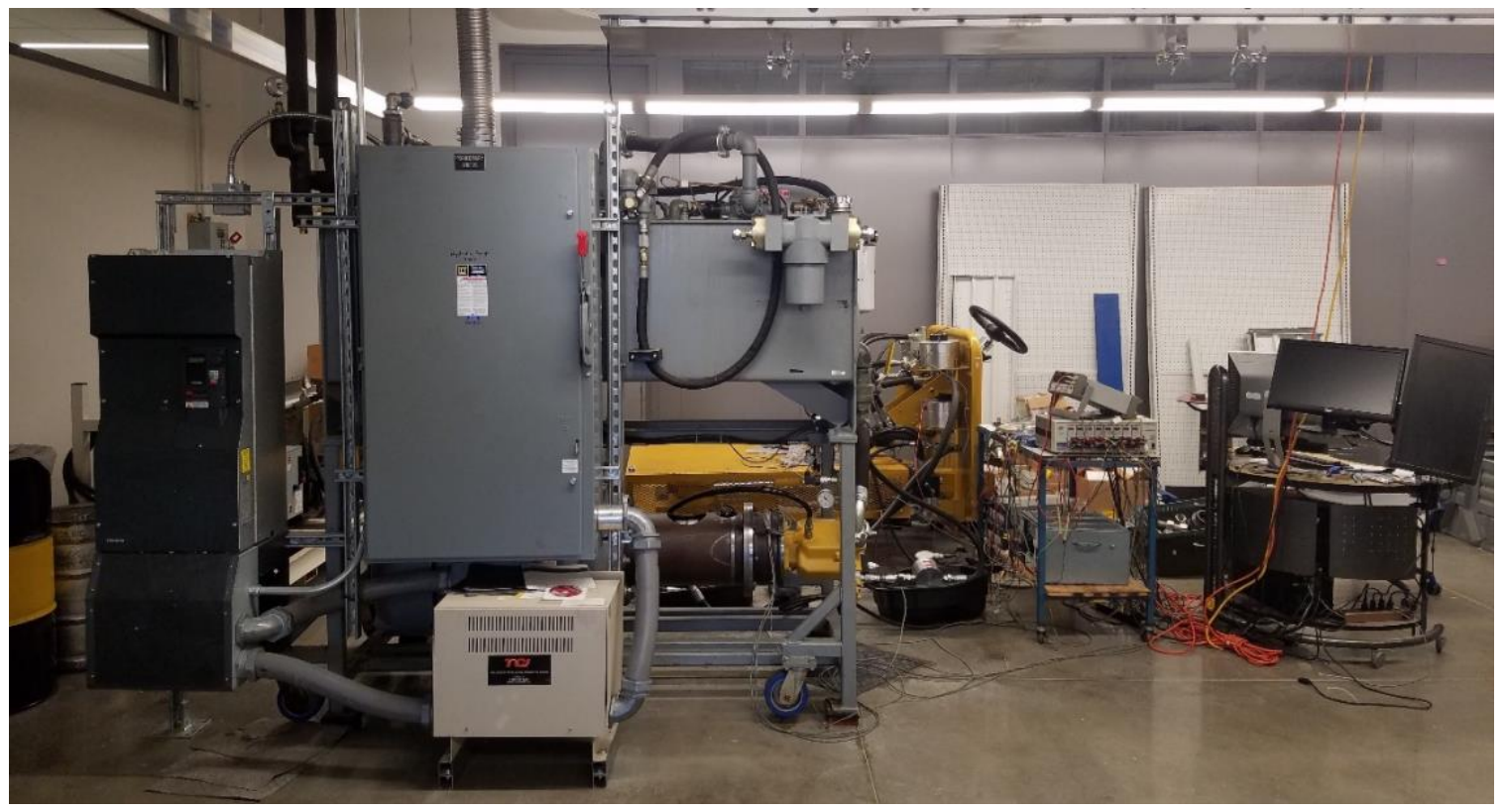

Figure 32. Experimental setup.

The experimental setup was also described in section 2.2 , but is repeated here including sensor setup. Figures 1-5 show detailed photos of the experimental setup. The setup consists of a 200-horsepower electric motor turning the axial piston pump shaft. Above the pump a 150 gallon reservoir of hydraulic oil is stored. The inlet port of the pump pulls in fluid from the reservoir, and discharges flow at a much higher pressure, the discharge pressure. Discharge flow goes through a hydromechanically actuated steering valve. A second path to tank has been added to implement flow loss. The flow loss goes through a manually adjusted needle valve that can be adjusted to change the flow rate. The steering valve is actuated by a gerotor pump within the steering wheel valve assembly. Depending on the steering wheel direction, flow either flows into chamber A or chamber B of the two 
single ended cylinder rod system. The other chamber flows back to tank. A restriction solenoid controlled pressure reducing valve has been put on both chambers to induce a load in the system. Discharge pressure, control pressure, load pressure, swash plate, and left cylinder position are analog differential measurements. Sensors were calibrated to manual specifications. Due to the noise of the electric motor, each of these differential measurements are passed through an eighth order analog Bessel filter. The bandwidth frequency is shown in Table 2 .

Table 2. Sensor filter bandwidth frequency.

\begin{tabular}{cc}
\hline \hline Sensor & Bandwidth Frequency \\
\hline Discharge Pressure & $1 \mathrm{kHz}$ \\
Control Pressure & $2.5 \mathrm{kHz}$ \\
Load Pressure & $1 \mathrm{kHz}$ \\
Swash Plate Position & $10 \mathrm{kHz}$ \\
Left Cylinder Position & $40 \mathrm{~Hz}$ \\
\hline
\end{tabular}

The discharge flow, return flow, discharge flow loss, and steering wheel velocity were measured through digital counter timers, and calibrated to manual specifications. 


\section{CHAPTER 6: EXPERIMENTAL RESULTS AND DISCUSSION}

\subsection{First Order Model Results}

The motor was operated at 750 RPM. The steering system was turned a set distance, and then turned back the same distance. This turning motion was done twice. Once the first full turning motion had been completed, the flow loss valve was opened prior to the next turning motion. Figure 33 shows the measured discharge pressure, load pressure, steering position, flow loss valve flow, and swash plate angle.
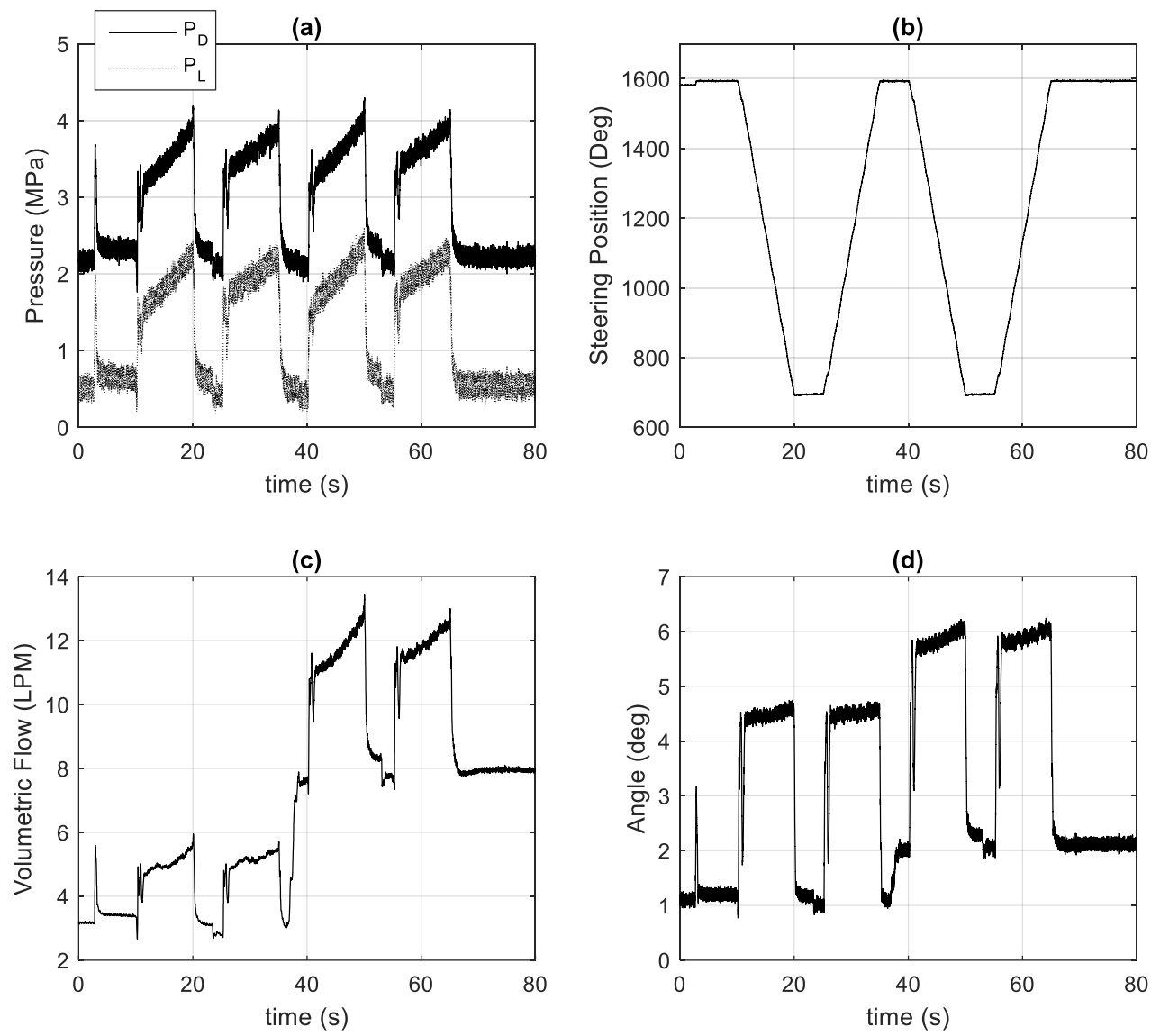

Figure 33. (a) Discharge pressure and load pressure, (b) steering position, (c) flow loss valve flow, and (d) swash plate angle measurements from experimental testing. 
The flow loss control valve was opened further at approximately 38 seconds. Figures 28 38 show the estimation of the PPF, KF, EKF, UFK, and a comparison between all four, respectively. Note that $K_{2}$ has been scaled.
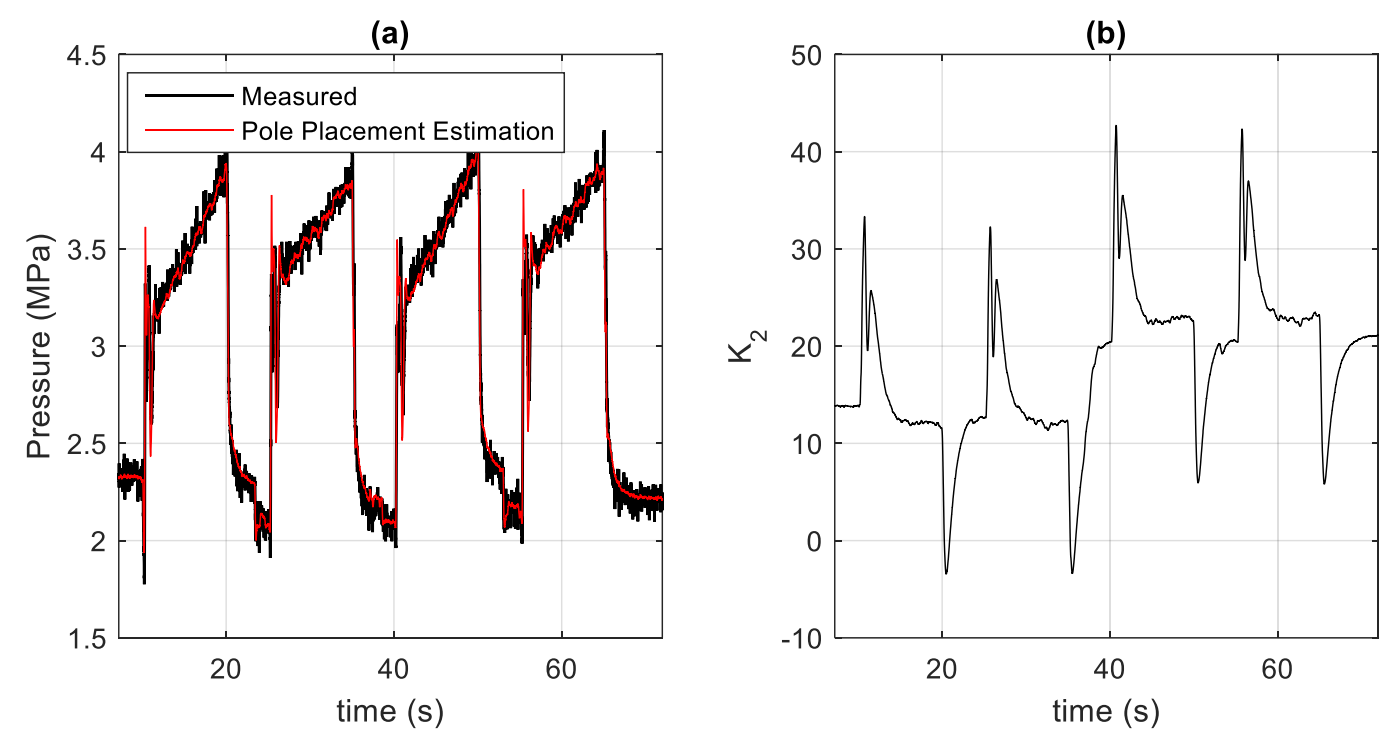

Figure 34. PPF (a) state and (b) parameter estimation.
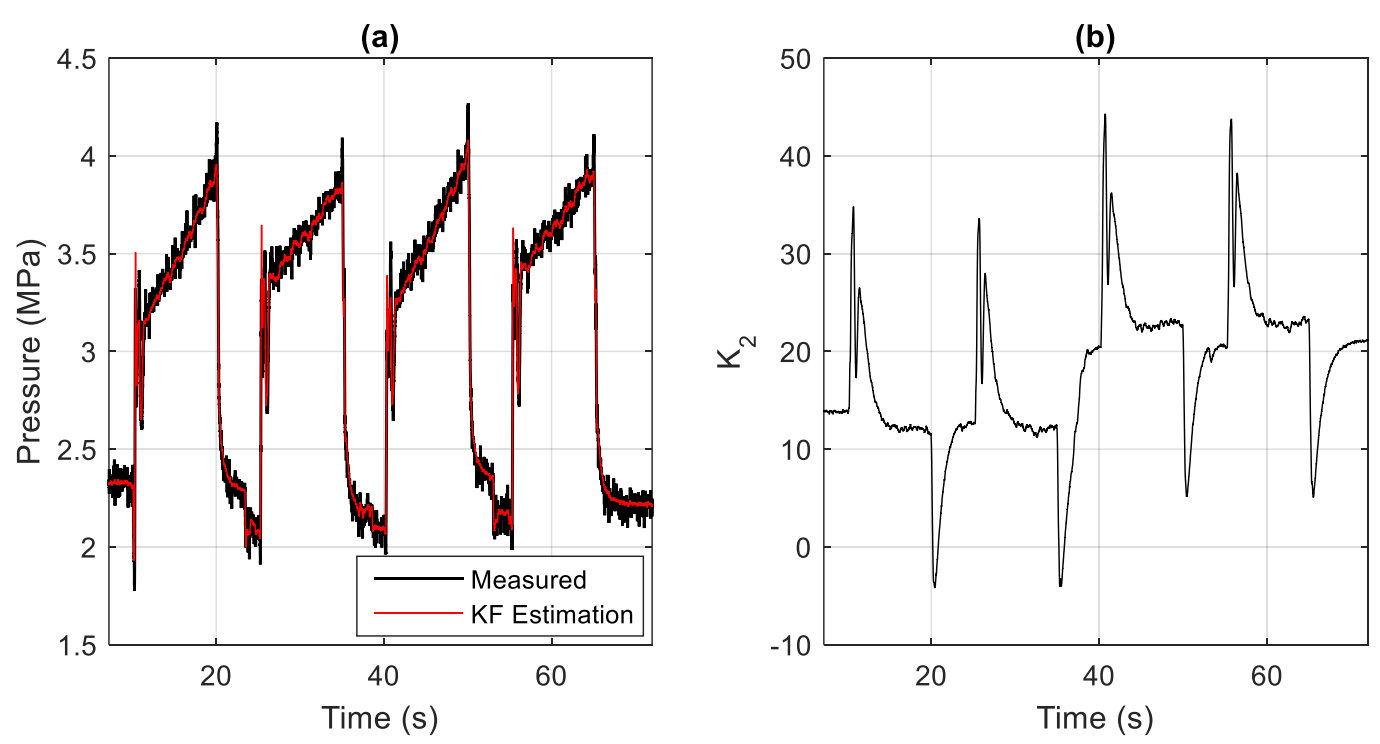

Figure 35. KF (a) state and (b) parameter estimation. 
(a)

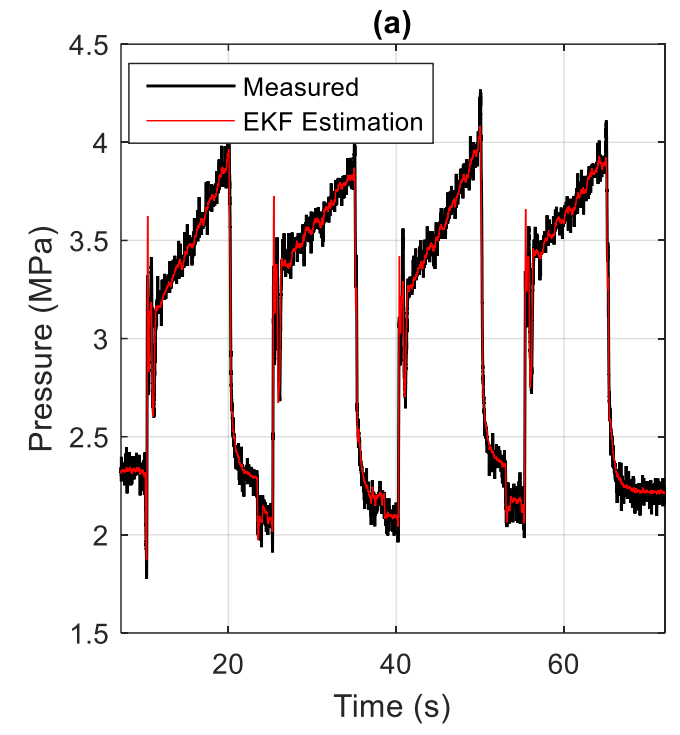

(b)

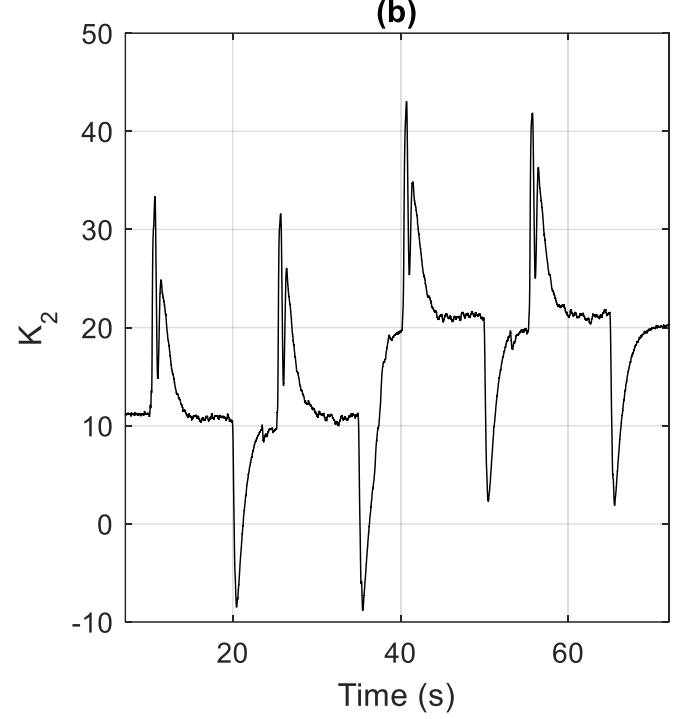

Figure 36. EKF (a) state and (b) parameter estimation.
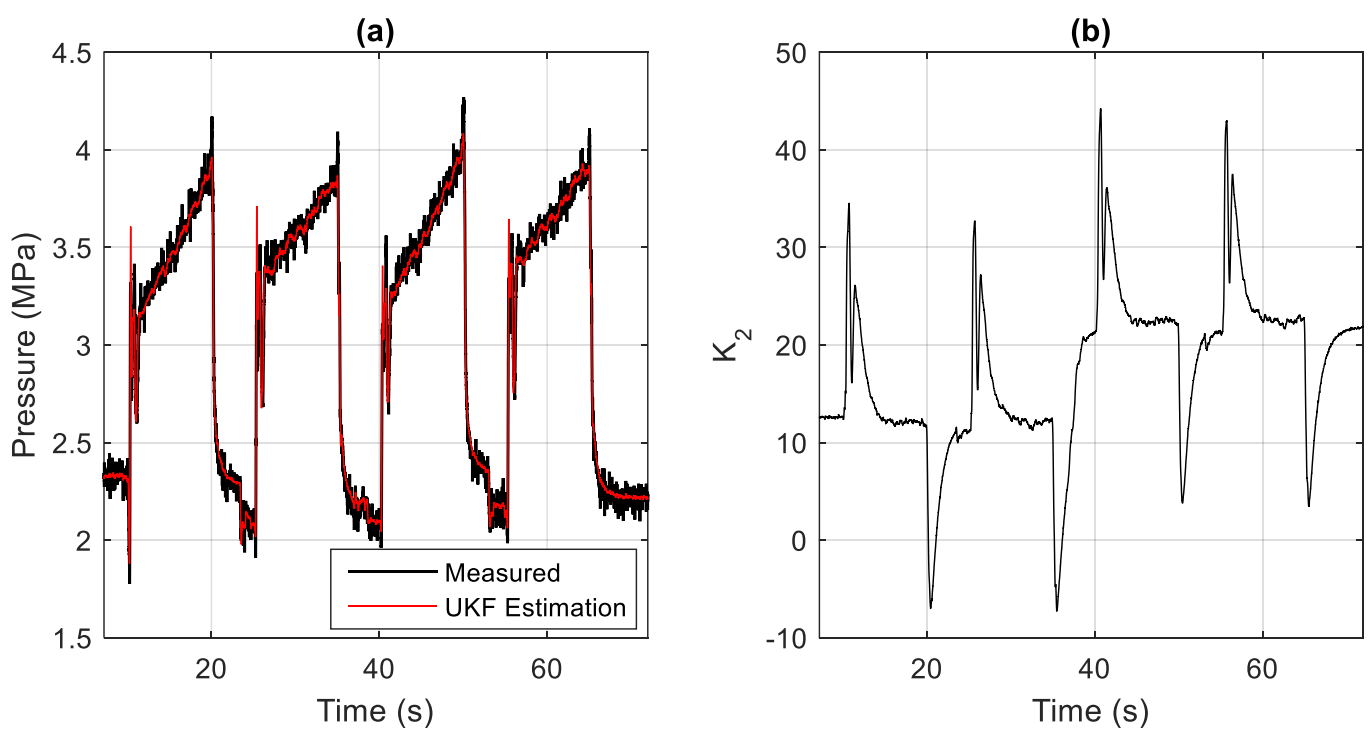

Figure 37. UKF (a) state and (b) parameter estimation. 
(a)

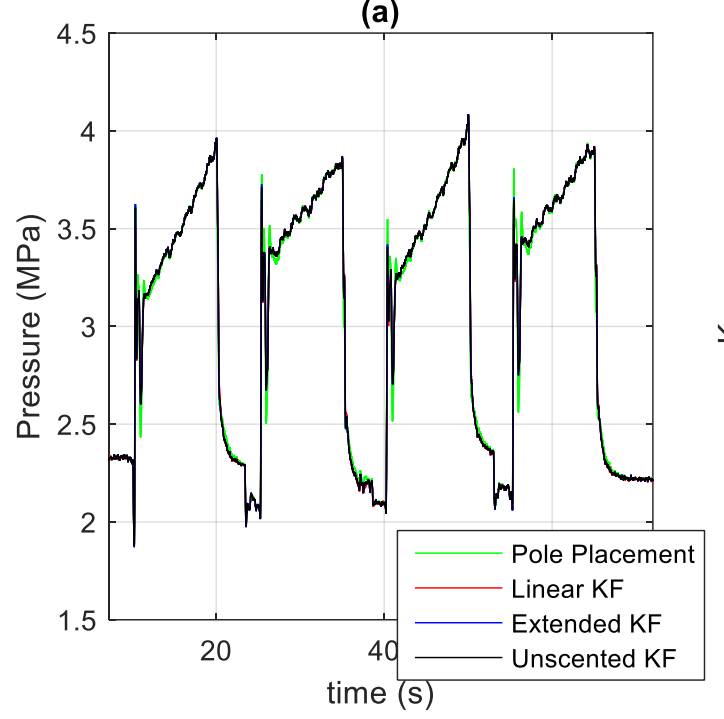

(b)

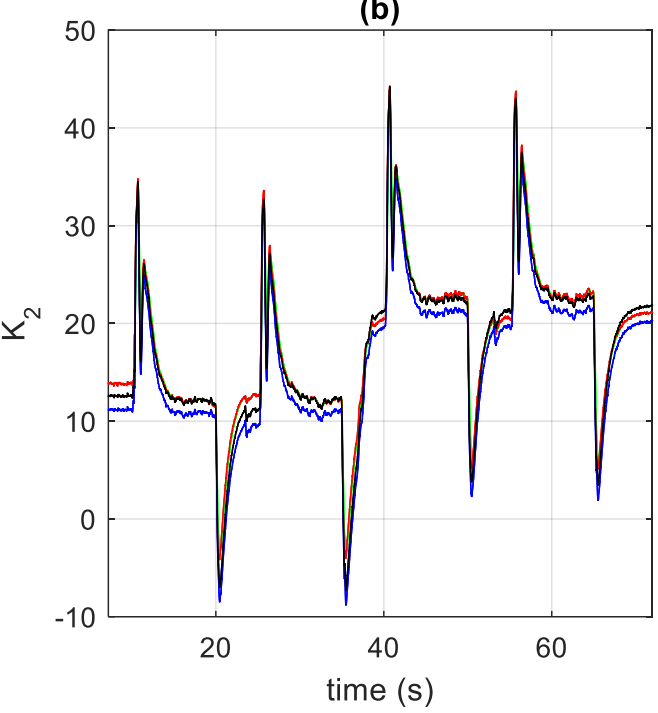

Figure 38. Comparison of all four filters for (a) state and (b) parameter estimation.

The performance of each filter is highly similar. Figures 28-38 show that every filter can filter out some of the measurement noise of the discharge pressure and has accurate estimation. The PPF has a slight difference in the discharge pressure estimation compared to the other three, but only during the large, rapid changes when the steering first begins. The flow loss coefficient estimation is also similar for all four filters. Note that the PPF and the KF, the two linear filters, estimation of the flow loss coefficient is highly dependent on the chosen nominal value for the flow loss coefficient. Choosing a different nominal value shifts the estimation, though the shape of the estimation remains the same. These results are encouraging. However, there is no way to determine the correct $K_{2}$ from these results. The testing is not steady state, so the general least squares fit should not be calculated to compare to the results. A simple PI controller was implemented to control the solenoid current in the control valves to keep the load pressure at a reference value steady during turning, which would also create a steady discharge pressure. The controller was 
tuned through trial and error. Testing was then redone. A simplified control feedback loop for the PI controller is shown in Fig. 39.

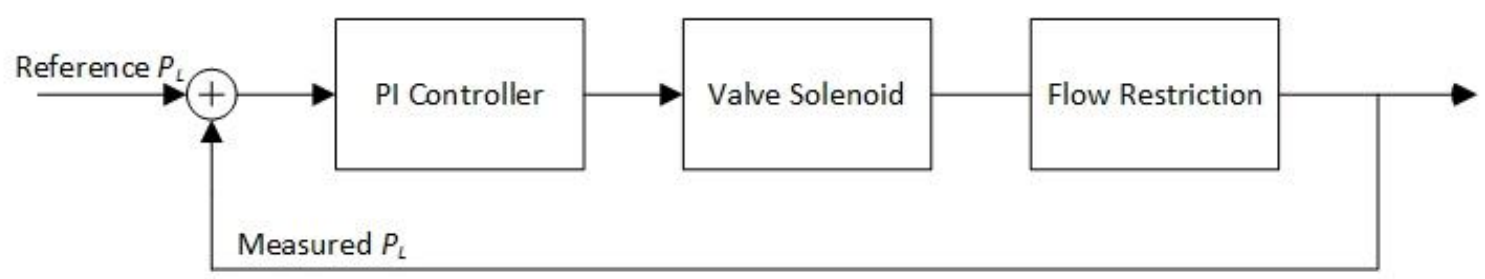

Figure 39. Simplified PI control feedback.

The testing with the load controller was done at multiple operating conditions. Figure 40 shows one such testing. The steering wheel is automatically controlled to turn at a steady rate, and then turned the opposite direction at the same speed for the same amount of time. The speed is then increased, and the turning profile is repeated. The speed is increased again, and the turning profile is repeated. During these turns, the load pressure is held constant by the feedback control algorithm for the restriction valves. Other tests included different load pressure set points, different motor RPM, and different leakage valve openings. 
(a)

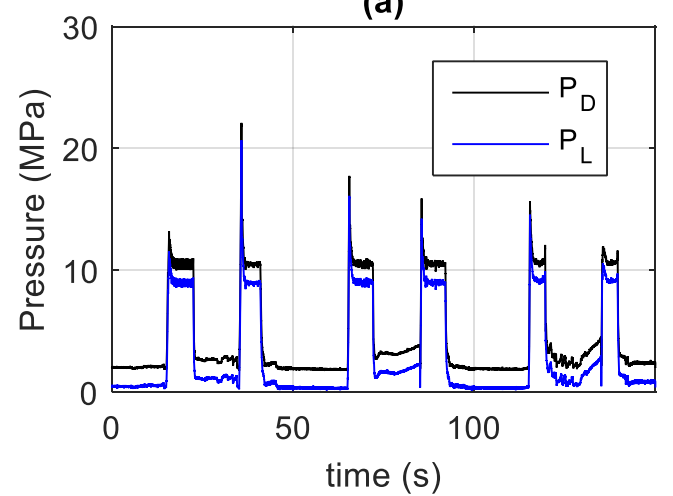

(c)

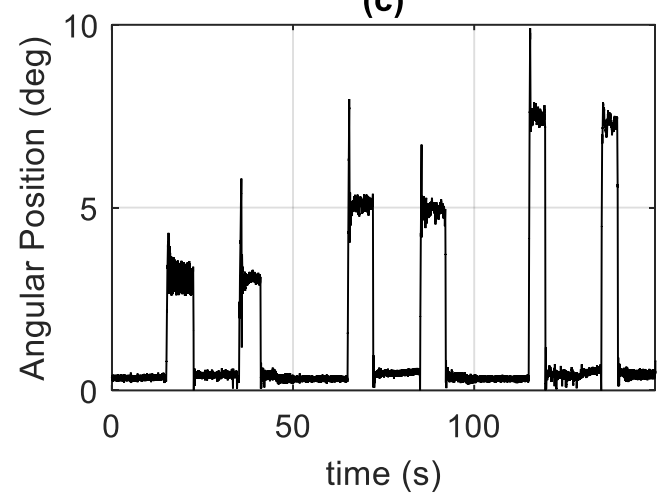

(e)

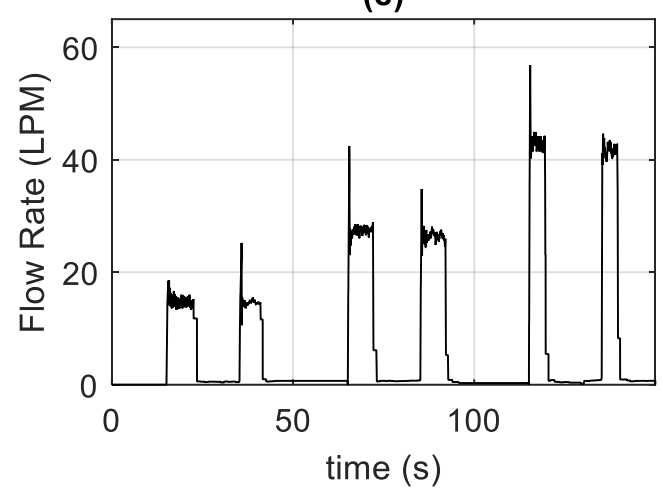

(b)

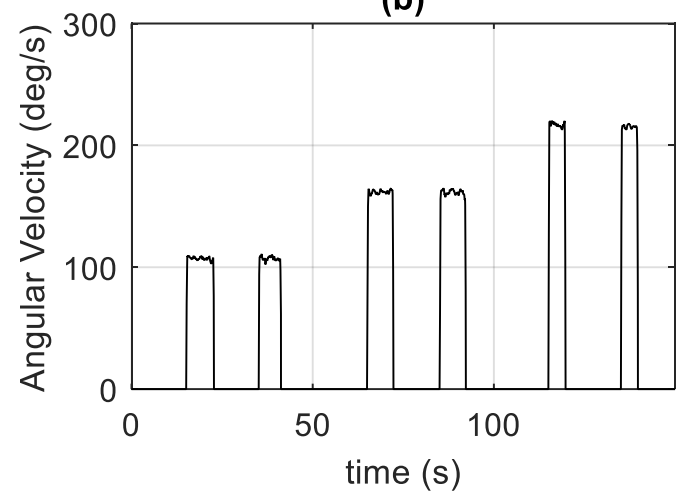

(d)

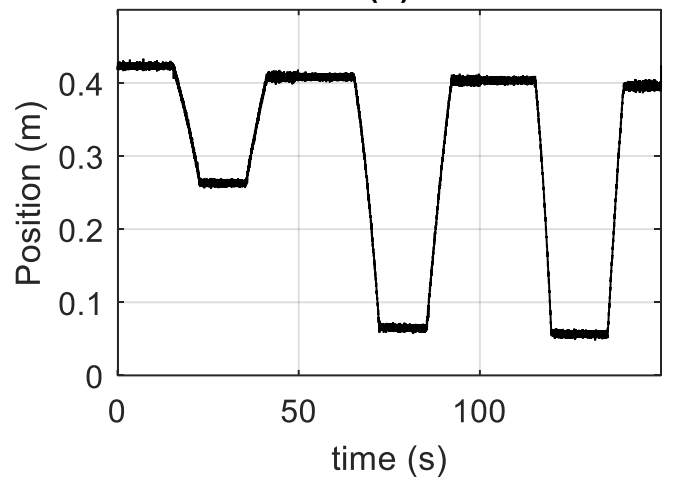

(f)

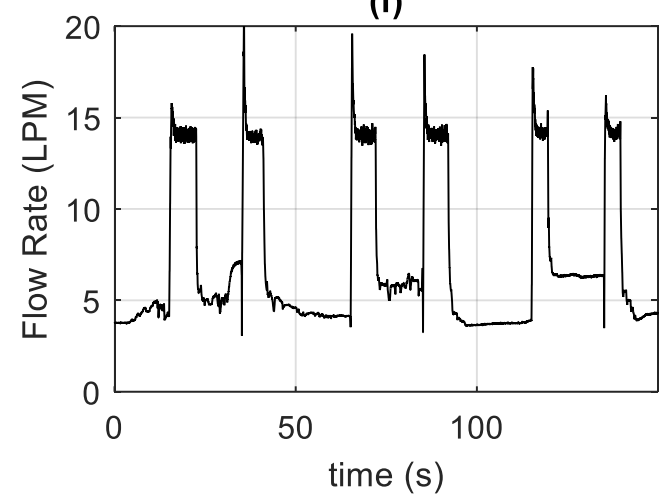

Figure 40. Experimental test for (a) discharge pressure (b) steering velocity (c) swash plate position (d) cylinder displacement (e) discharge flow Rate (f) leakage valve Flow Rate.

Experimental data was collected into three separate categories: the discharge flow loss valve closed, the discharge flow loss valve opened half a turn of the dial, and the discharge flow loss valve opened a full turn. For each category of data, the general least squares method was applied to data only during steering turning phases. The results of these fits can be seen in the table below. 
Table 3. General least square fit results.

\begin{tabular}{llll}
\hline Discharge Flow Loss Valve Position & $K_{1}$ & $K_{2}$ & $r^{2}$ \\
\hline Closed & $2.16 e^{-12}$ & $1.29 e^{-8}$ & 0.965 \\
Half Turn & $2.23 e^{-12}$ & $5.23 e^{-8}$ & 0.973 \\
Full Turn & $3.27 e^{-12}$ & $7.78 e^{-8}$ & 0.979 \\
\hline \hline
\end{tabular}

The EKF was ran at $100 \mathrm{~Hz}$ for the first order model for the testing described above. The states, measurements, and inputs were

$$
x=\left[\begin{array}{c}
P_{D} \\
K_{2}
\end{array}\right], \quad \mathrm{z}=\mathrm{P}_{\mathrm{D}}, \quad \text { and } u=\left[\begin{array}{c}
u_{w} \\
\alpha \\
P_{L}
\end{array}\right] .
$$

For all experimental results, the process and measurement noise was chosen with some knowledge of the system. The standard deviation of the measurements could be determined for the measurement noise directly and was then tuned to further improve the filter. The process noise was initially chosen to be large to give a conservative estimation, and then was further tuned to improve estimation. The process noise and measurement noise was

$$
v(t)=\left[\begin{array}{c}
1.73 e^{5} \\
0.27
\end{array}\right] \text { and } n(t)=1.73 e^{5}
$$




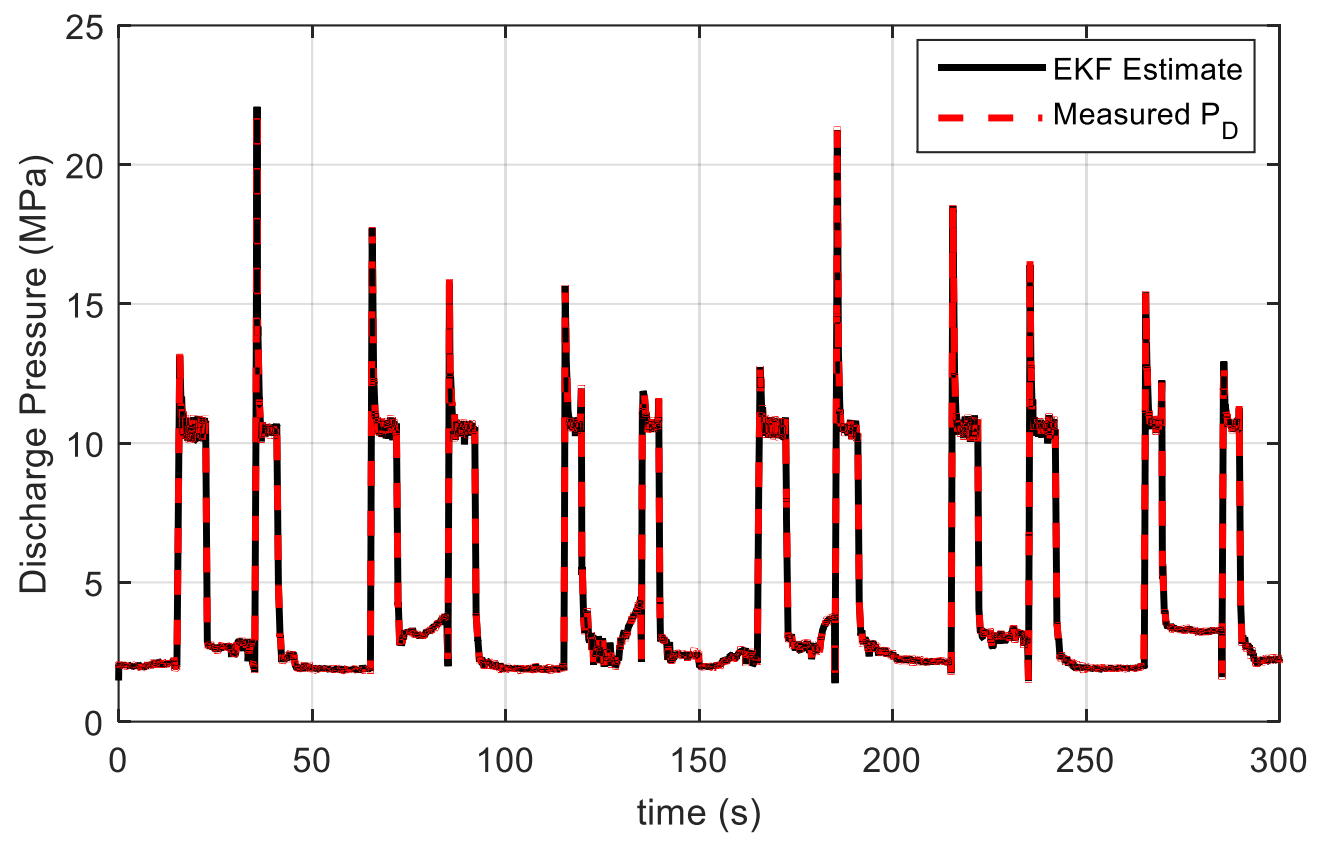

Figure 41. Discharge pressure estimation from single state model utilizing the EKF.

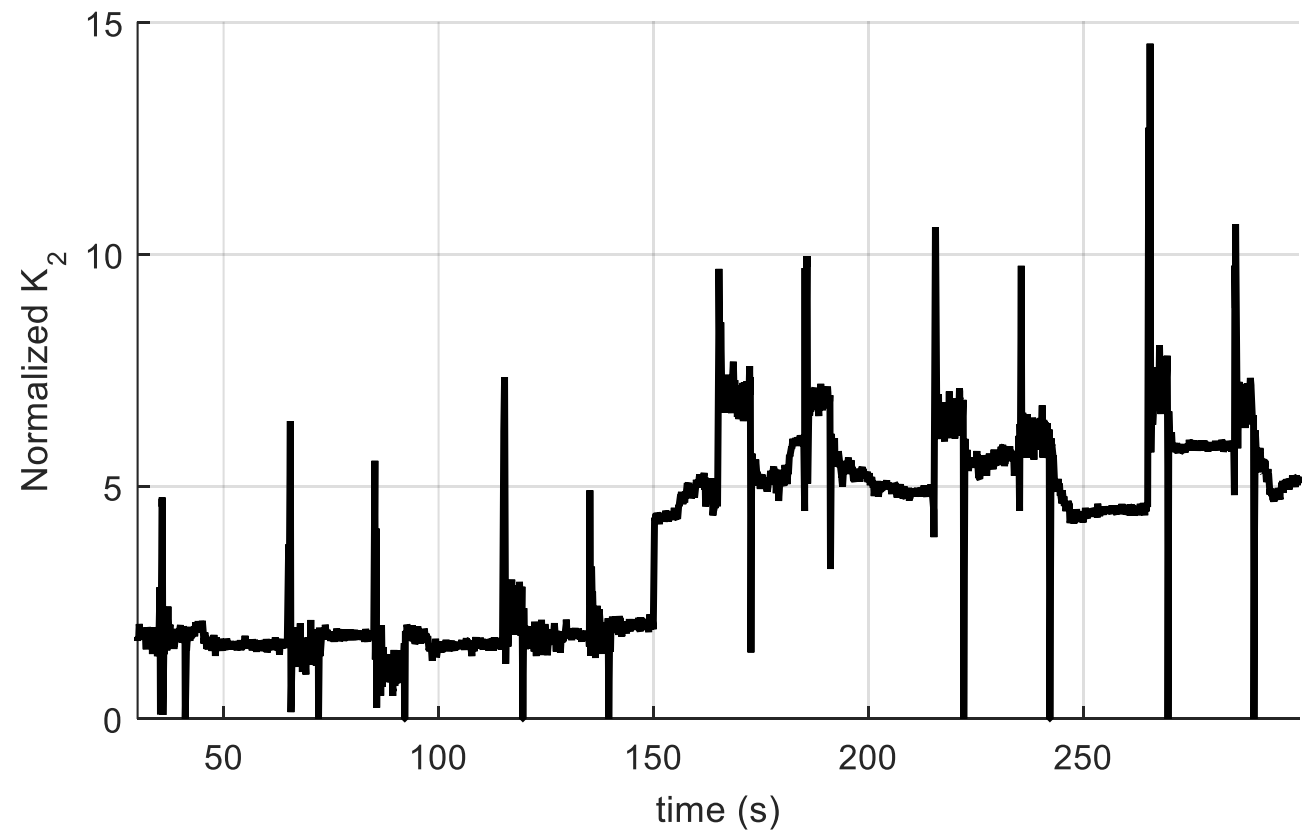

Figure 42. Flow loss coefficient estimation from single state model utilizing the EKF. 


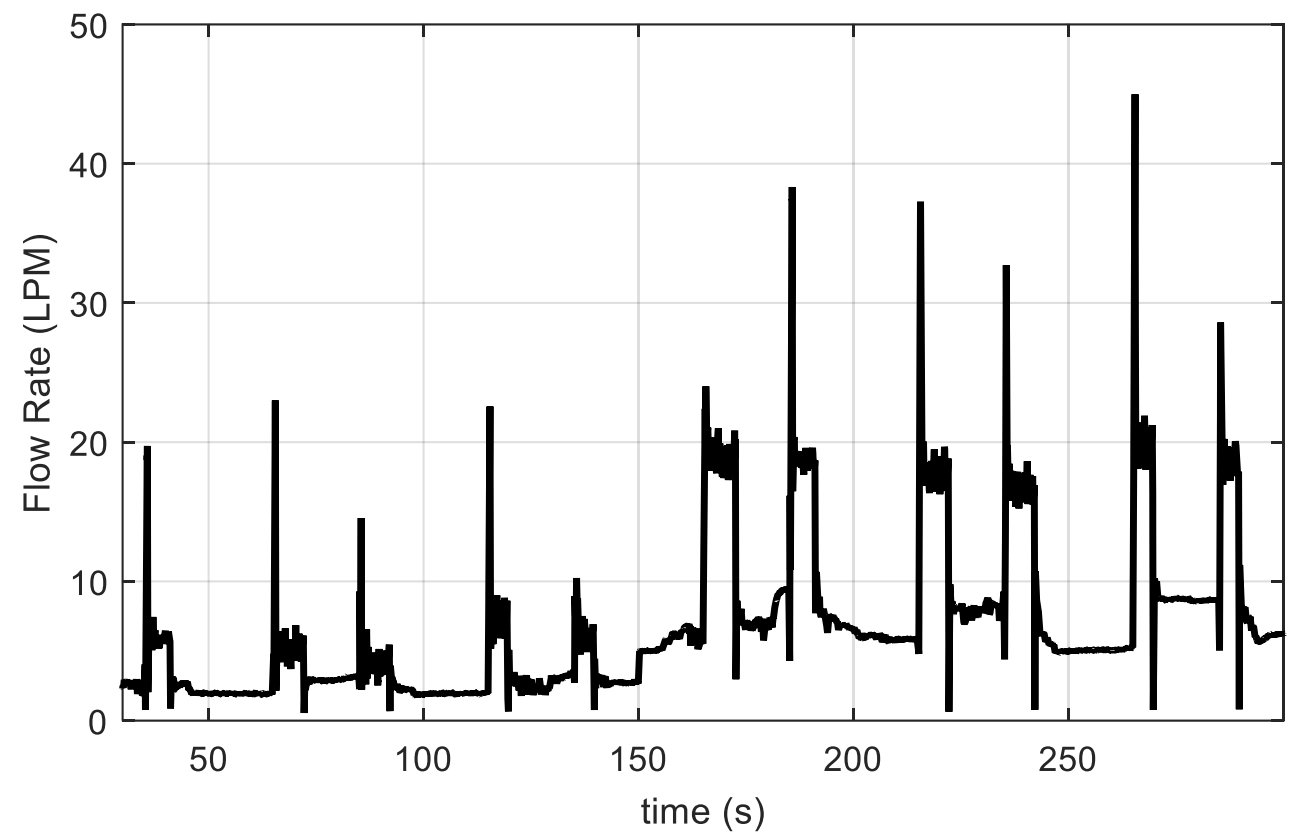

Figure 43. Flow loss estimation from single state model utilizing the EKF.

The discharge pressure measurement matches up well with the estimation. The flow loss coefficient increases where the flow loss valve is opened, resulting in a higher flow loss estimation. The first order results are compared to the second order results in the next section.

\subsection{Second Order Model Results}

The EKF was then ran on the second order model at $100 \mathrm{~Hz}$, to see if modeling the swash plate angle improved estimation. The states, measurements, and inputs were

$$
x=\left[\begin{array}{c}
P_{D} \\
\alpha \\
K_{2}
\end{array}\right], \quad \mathrm{z}=\left[\begin{array}{c}
\mathrm{P}_{\mathrm{D}} \\
\alpha
\end{array}\right], \quad \text { and } u=\left[\begin{array}{c}
u_{w} \\
P_{L}
\end{array}\right] .
$$

The high Reynolds number parameter was estimated for this flow loss model. The process and measurement noise was 


$$
v(t)=\left[\begin{array}{c}
1.73 e^{5} \\
2.66 e^{-3} \\
0.27
\end{array}\right] \text { and } n(t)=\left[\begin{array}{c}
1.73 e^{5} \\
2.66 e^{-3}
\end{array}\right]
$$

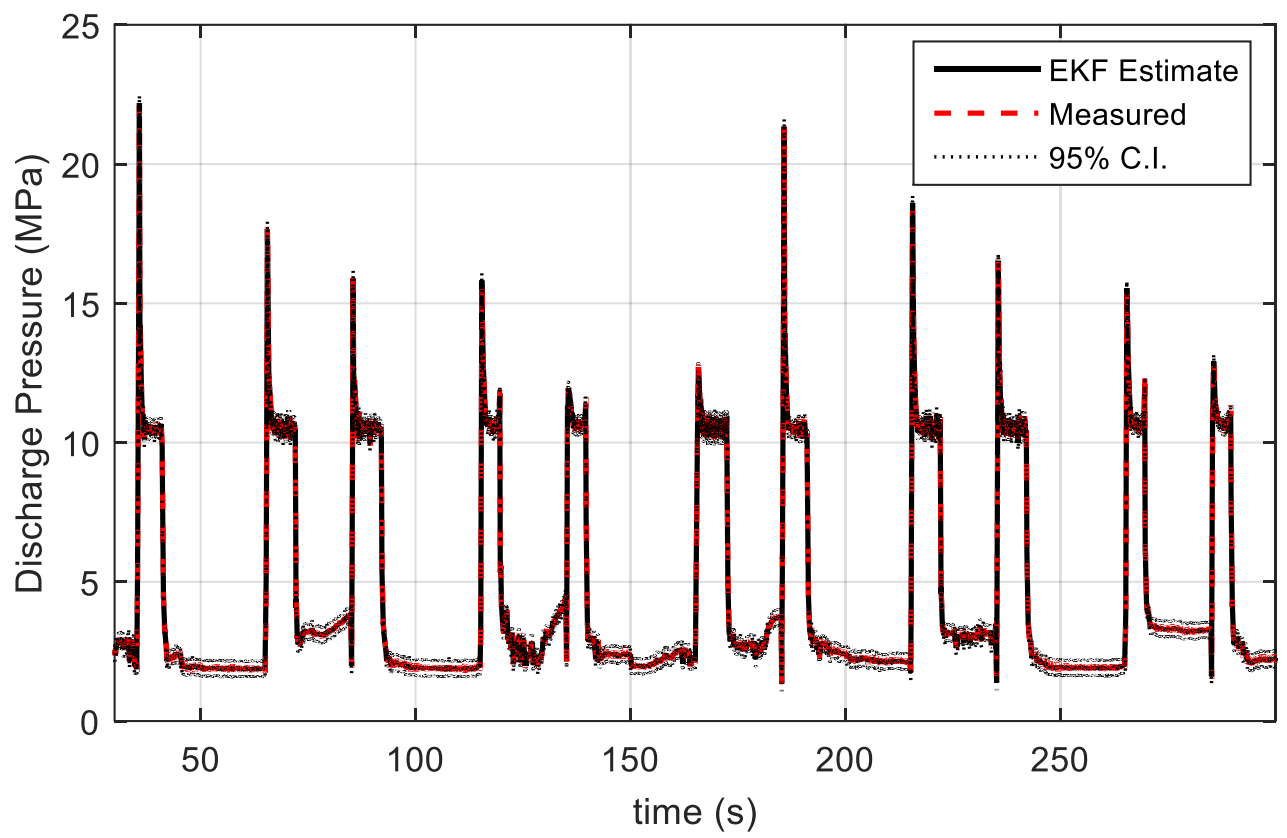

Figure 44. Discharge pressure estimation from the two state model utilizing the EKF. 


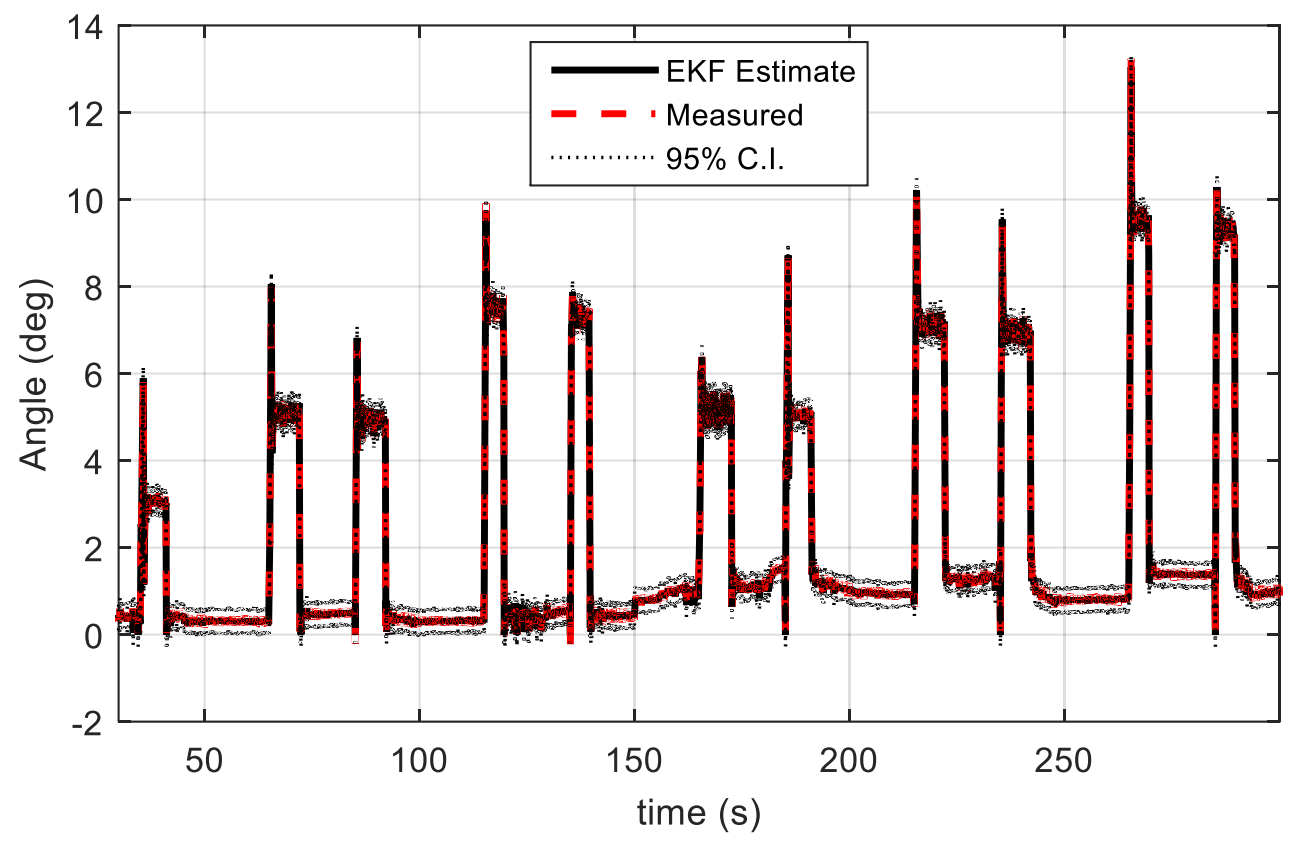

Figure 45. Swash plate angle estimation from the two state model utilizing the EKF.

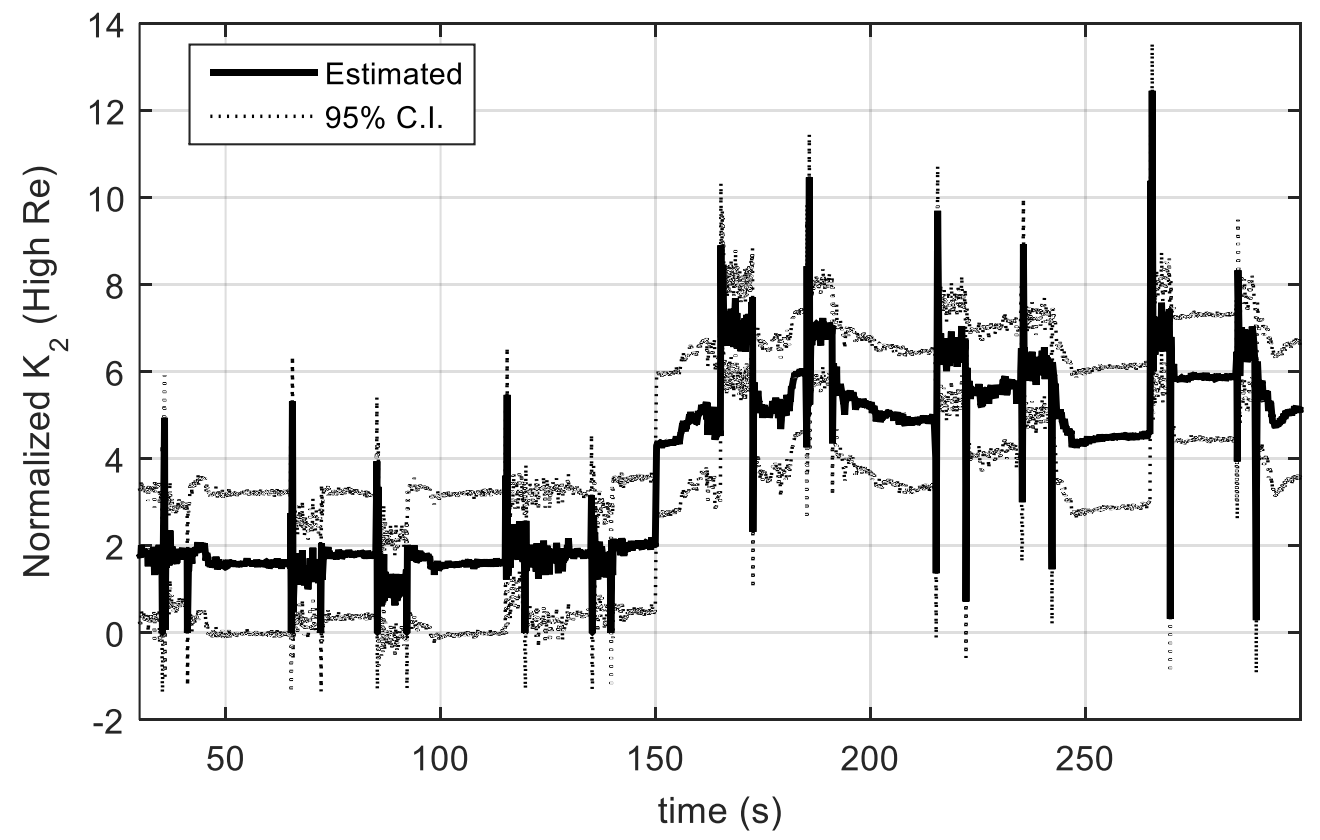

Figure 46. Flow loss coefficient estimation from the two state model utilizing the EKF. 


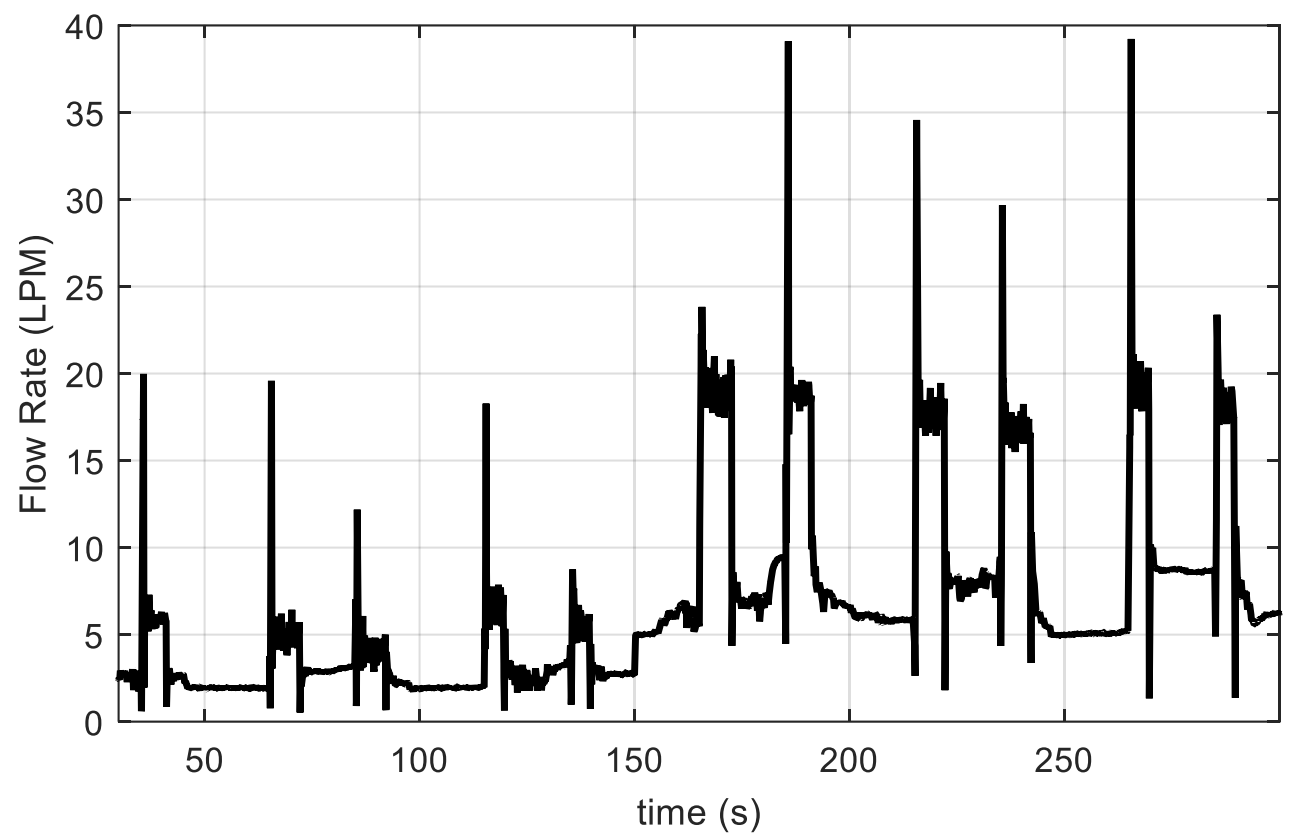

Figure 47. Flow loss estimation from the two state model utilizing the EKF.

The previous estimation was redone using the UKF.

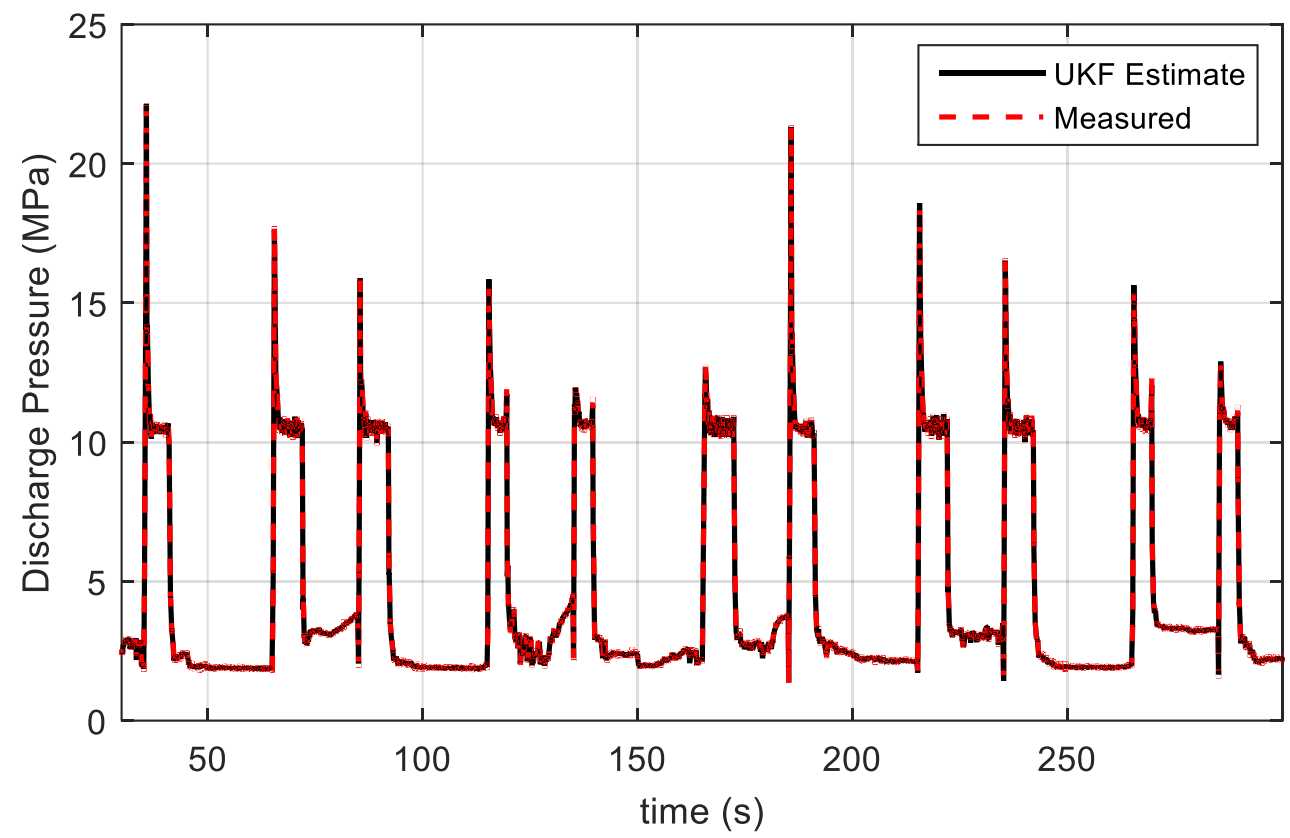

Figure 48. Discharge pressure estimation from the two state model utilizing the UKF. 


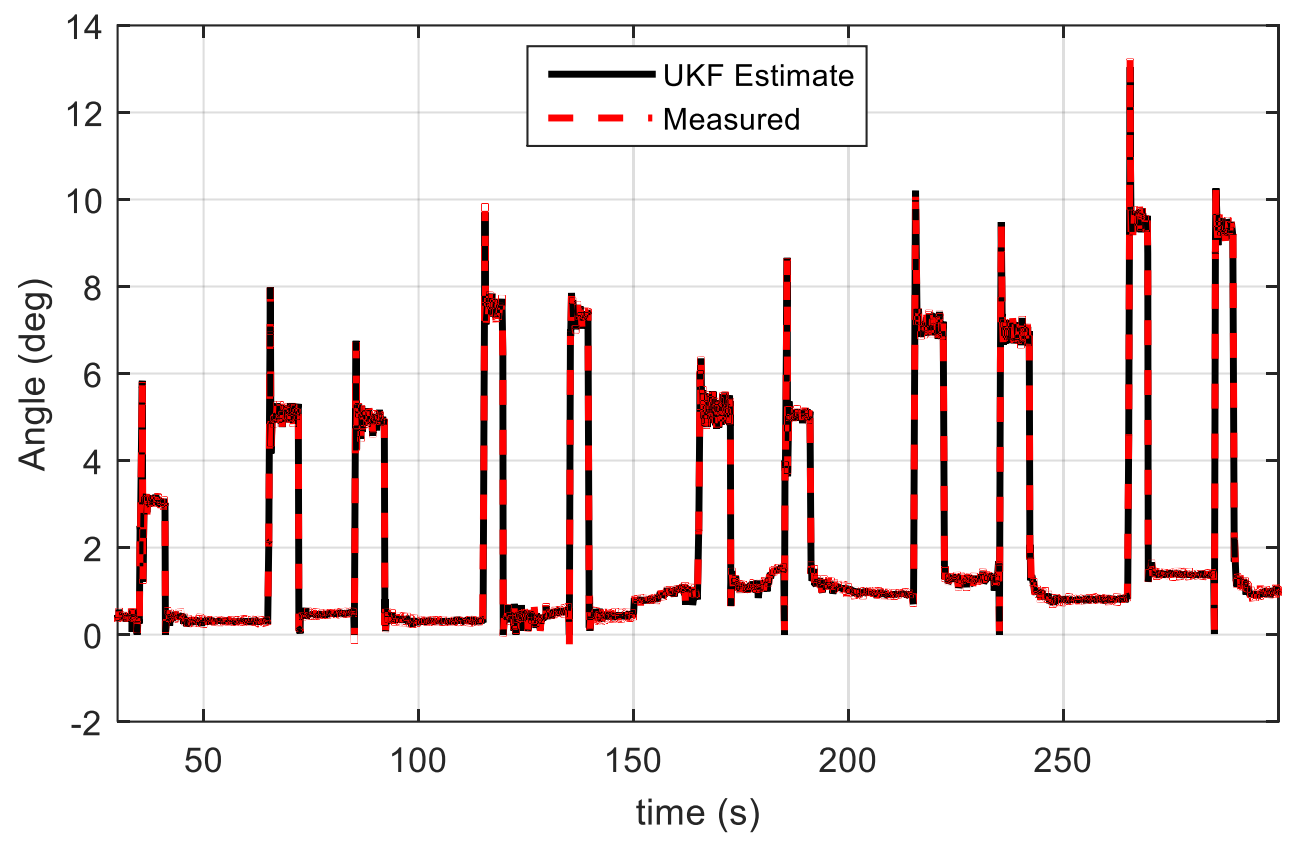

Figure 49. Swash plate angle estimation from the two state model utilizing the UKF.

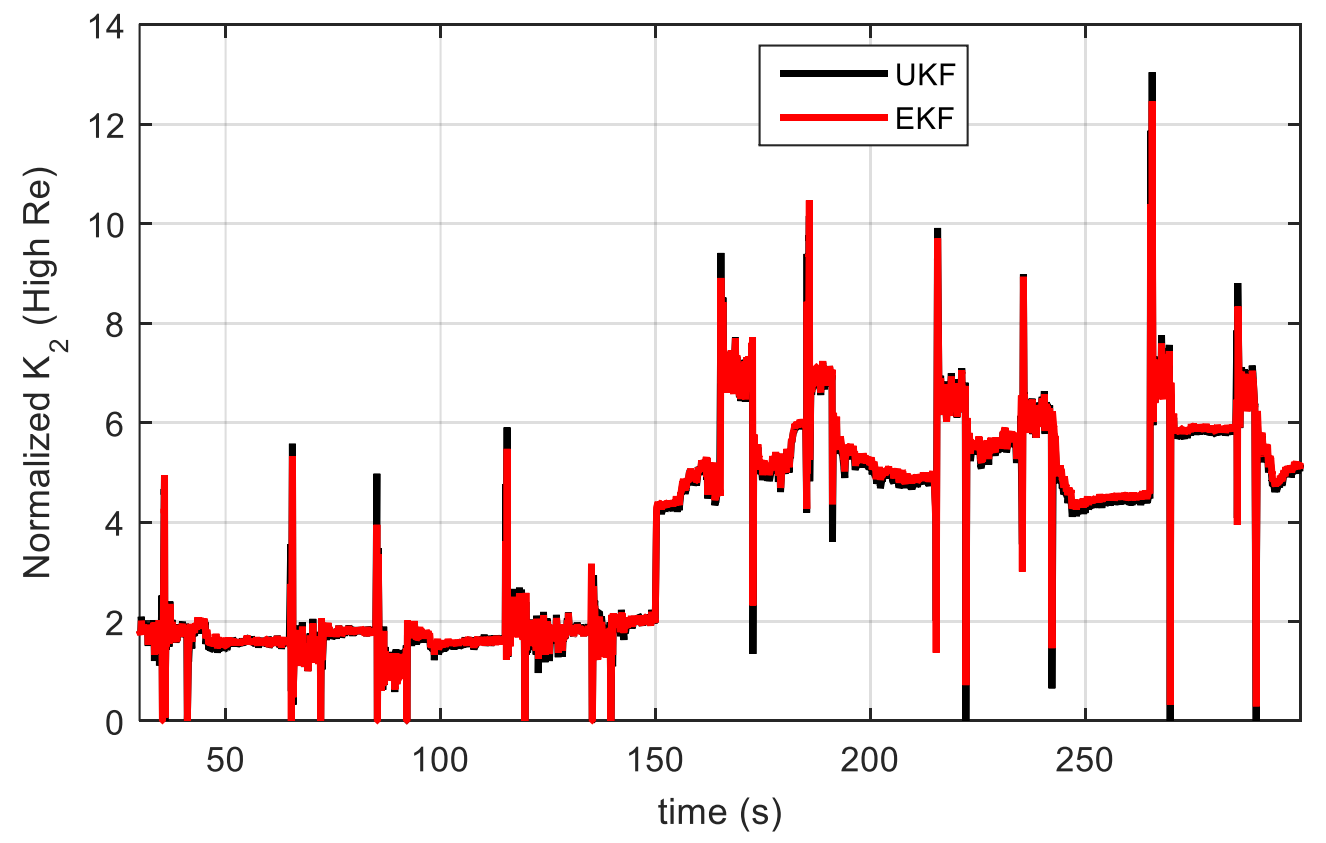

Figure 50. Comparison of EKF and UKF estimation of flow loss coefficient utilizing the two state model. 
There is no obvious improvement or benefit to the UKF for this work. The estimation for both state and parameter is extremely similar. Therefore, the decision was made to work solely with the EKF.

The EKF algorithm was also done for the scaled parameter estimation, $K_{3}$, and the offset parameter estimation, $K_{4}$. Figures 51-54 show the results for estimating $K_{3}$ and Figs. 55-58 show the results for estimating $K_{4}$. The process and measurement noise utilized above was used for these two results.

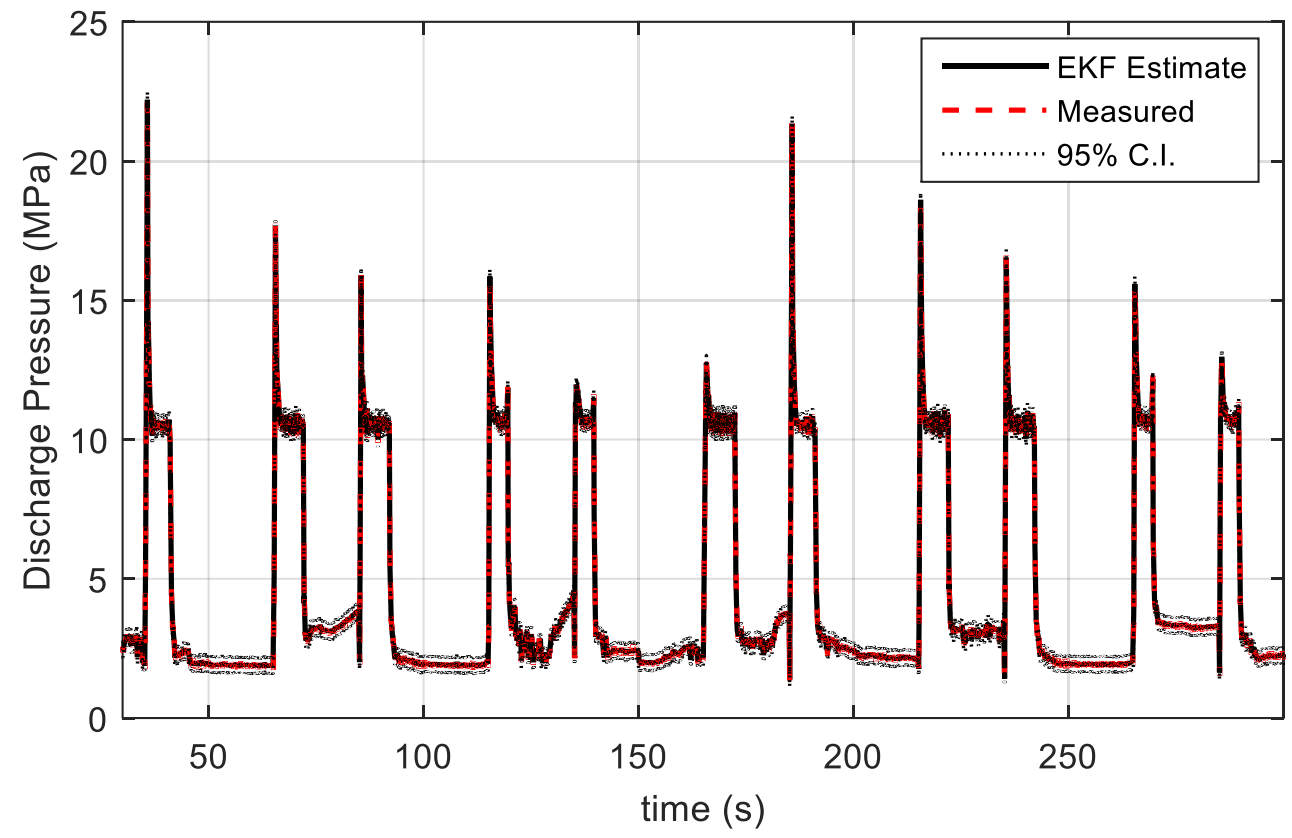

Figure 51. Discharge pressure estimation from the two state model utilizing the EKF with scaled flow loss model. 


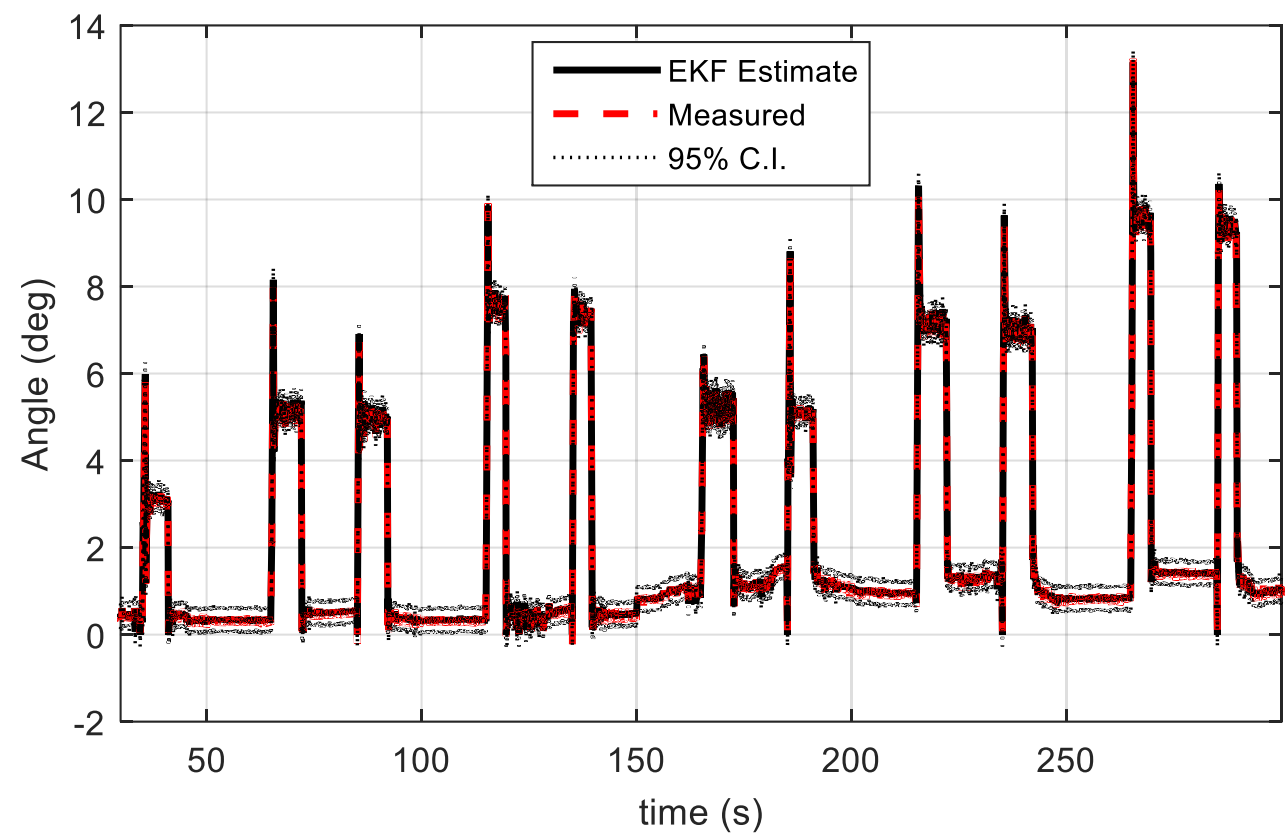

Figure 52. Swash plate angle estimation from the two state model utilizing the EKF with scaled flow loss model.

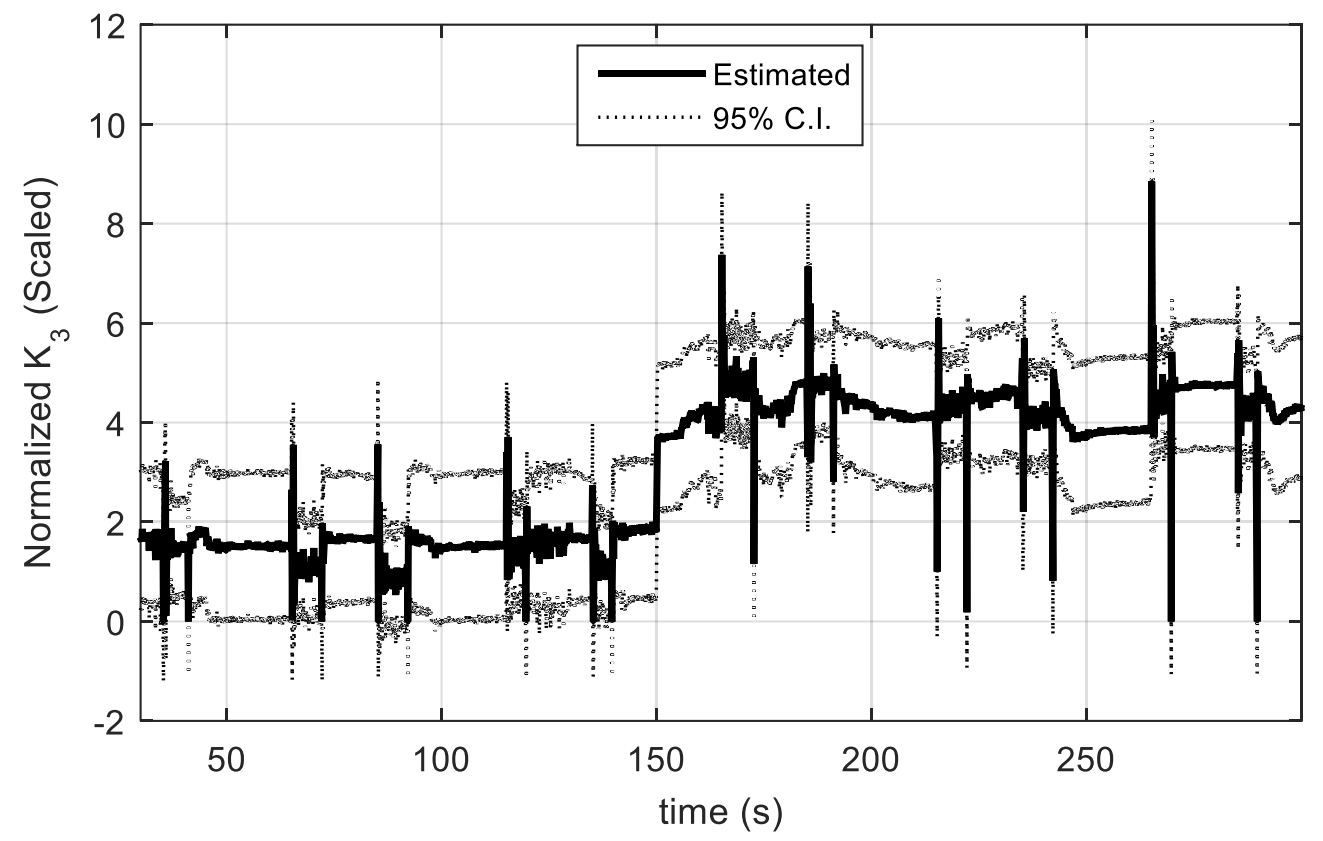

Figure 53. Flow loss coefficient estimation from the two state model utilizing the EKF with scaled flow loss model. 


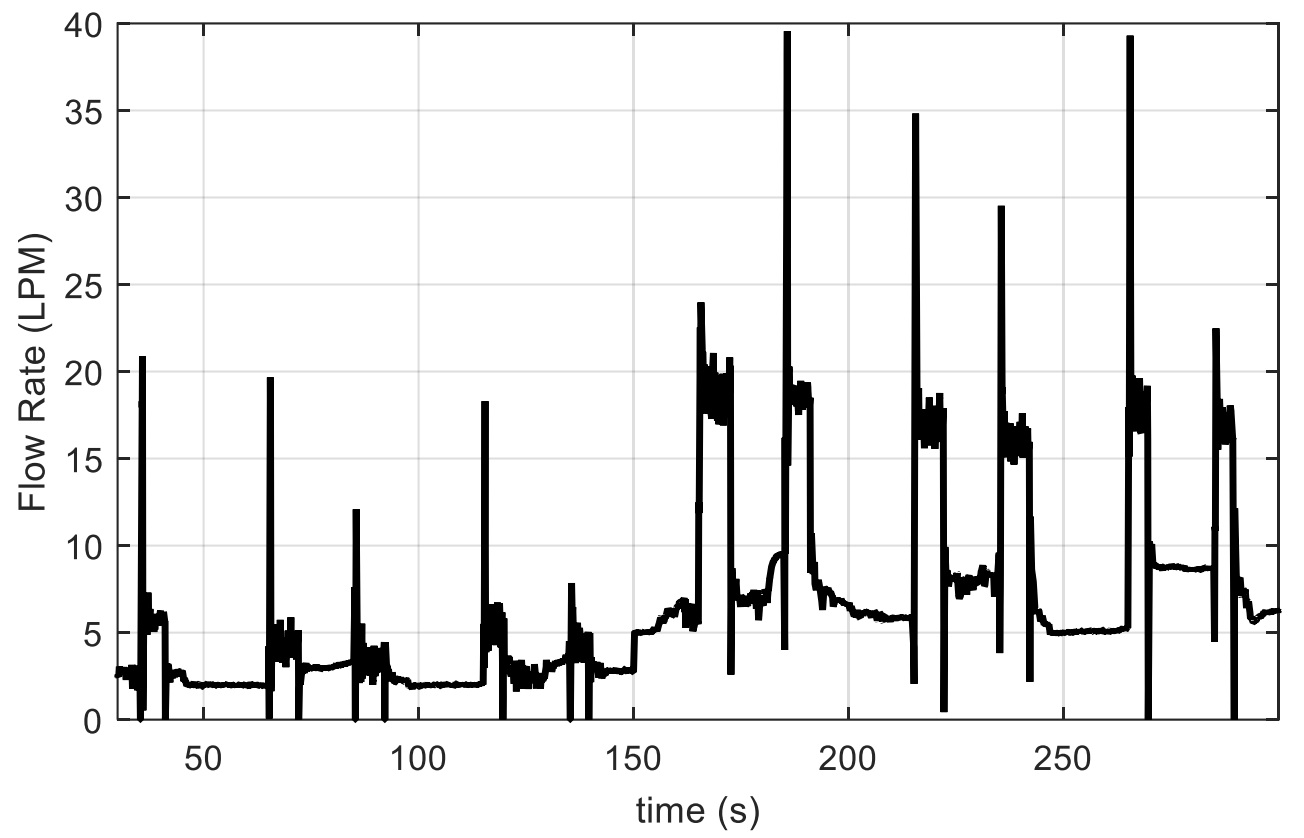

Figure 54. Flow loss estimation from the two state model utilizing the EKF with scaled flow loss model.

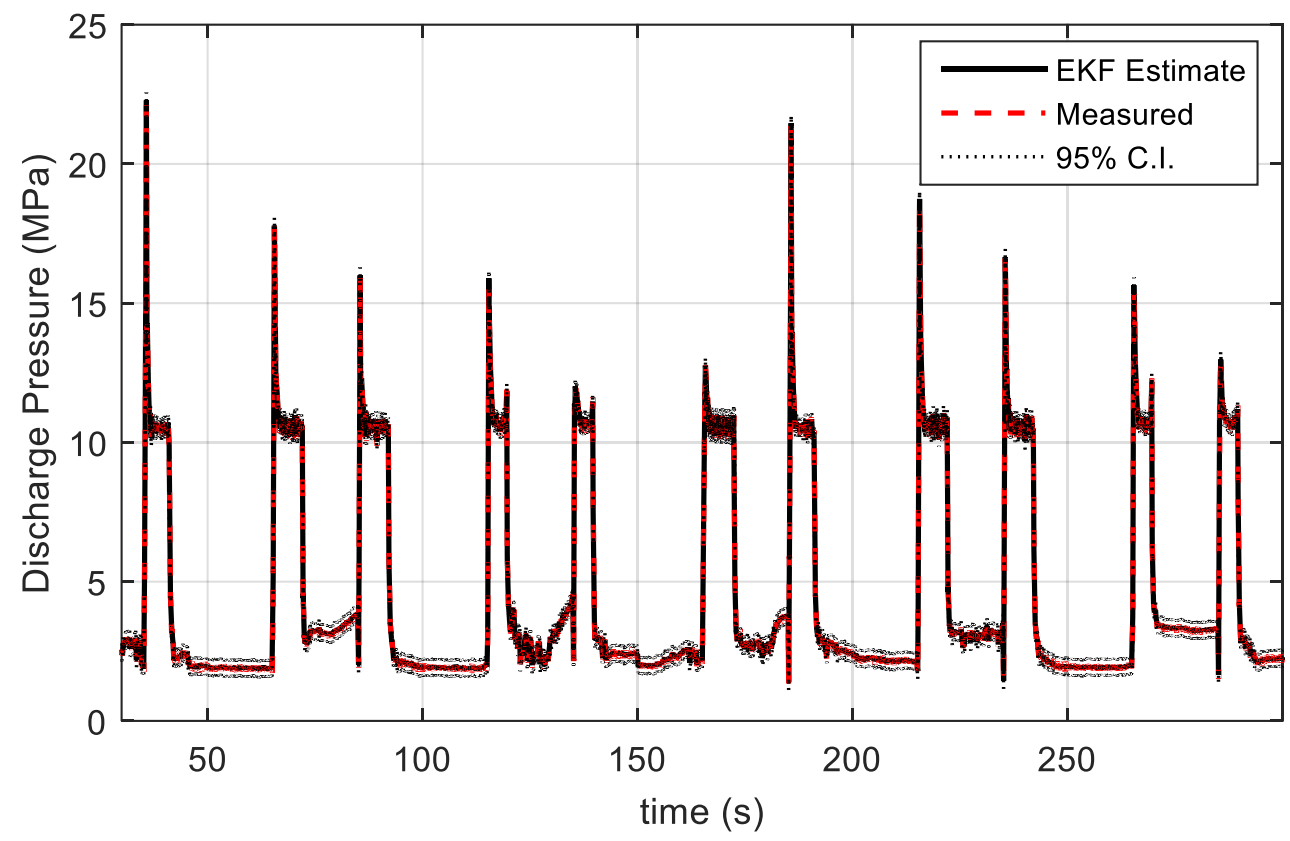

Figure 55. Discharge pressure estimation from the two state model utilizing the EKF with offset flow loss model. 


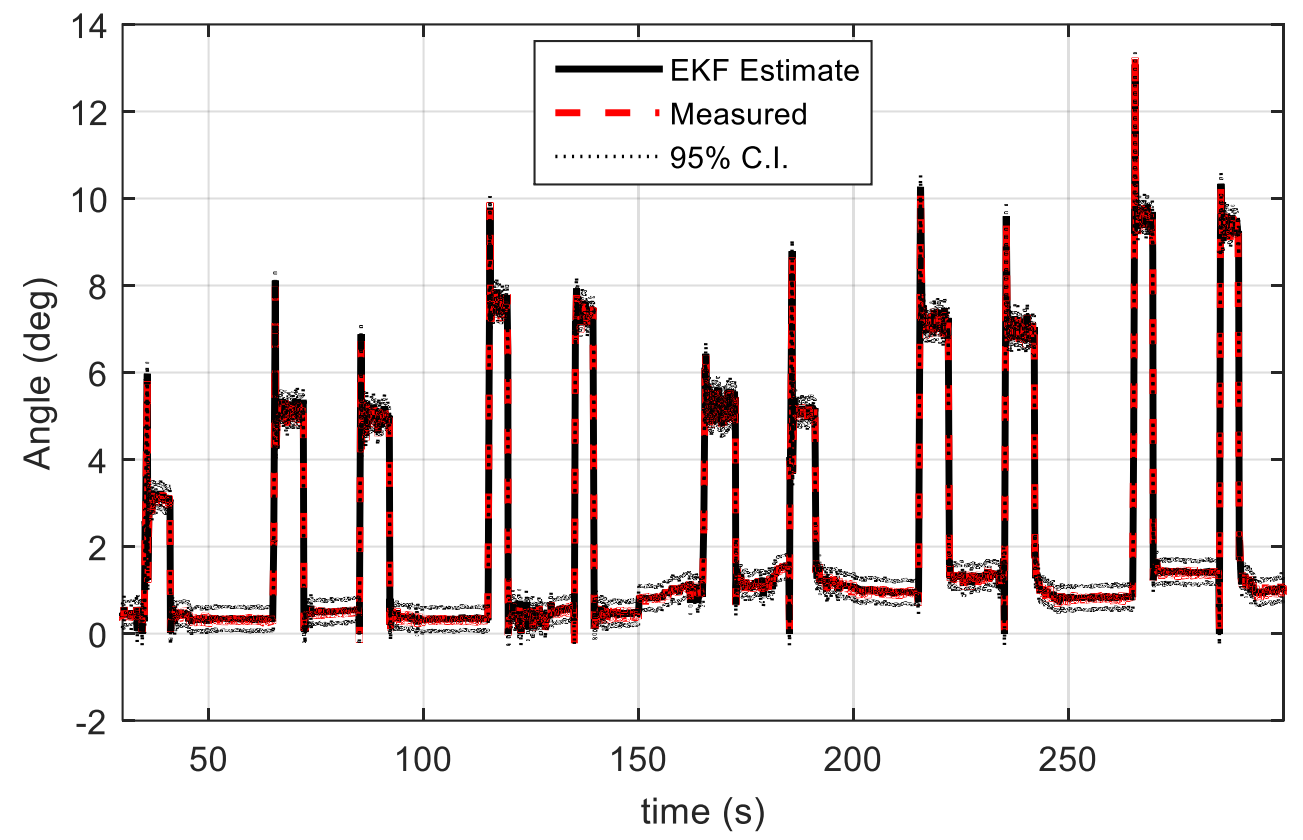

Figure 56. Swash plate estimation from the two state model utilizing the EKF with offset flow loss model.

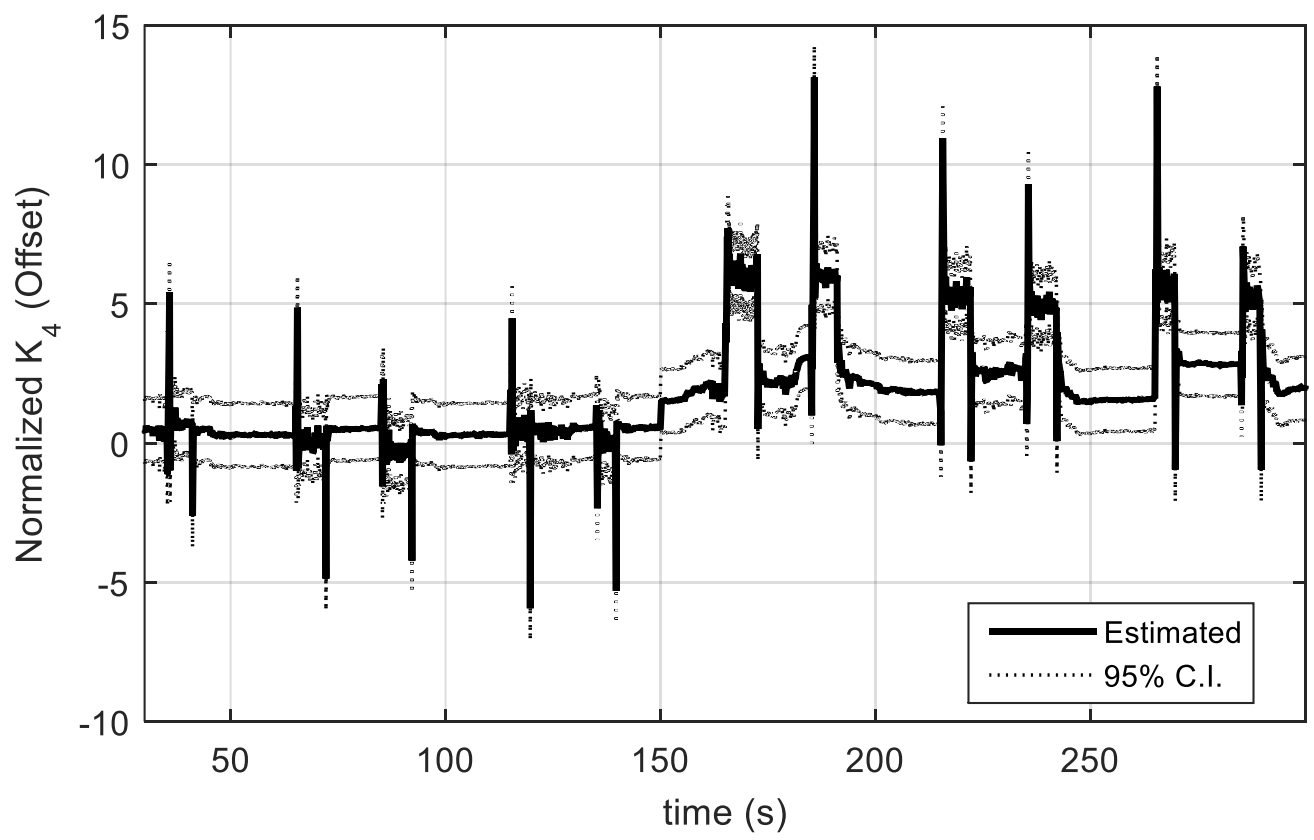

Figure 57. Flow loss coefficient estimation from the two state model utilizing the EKF with offset flow loss model. 


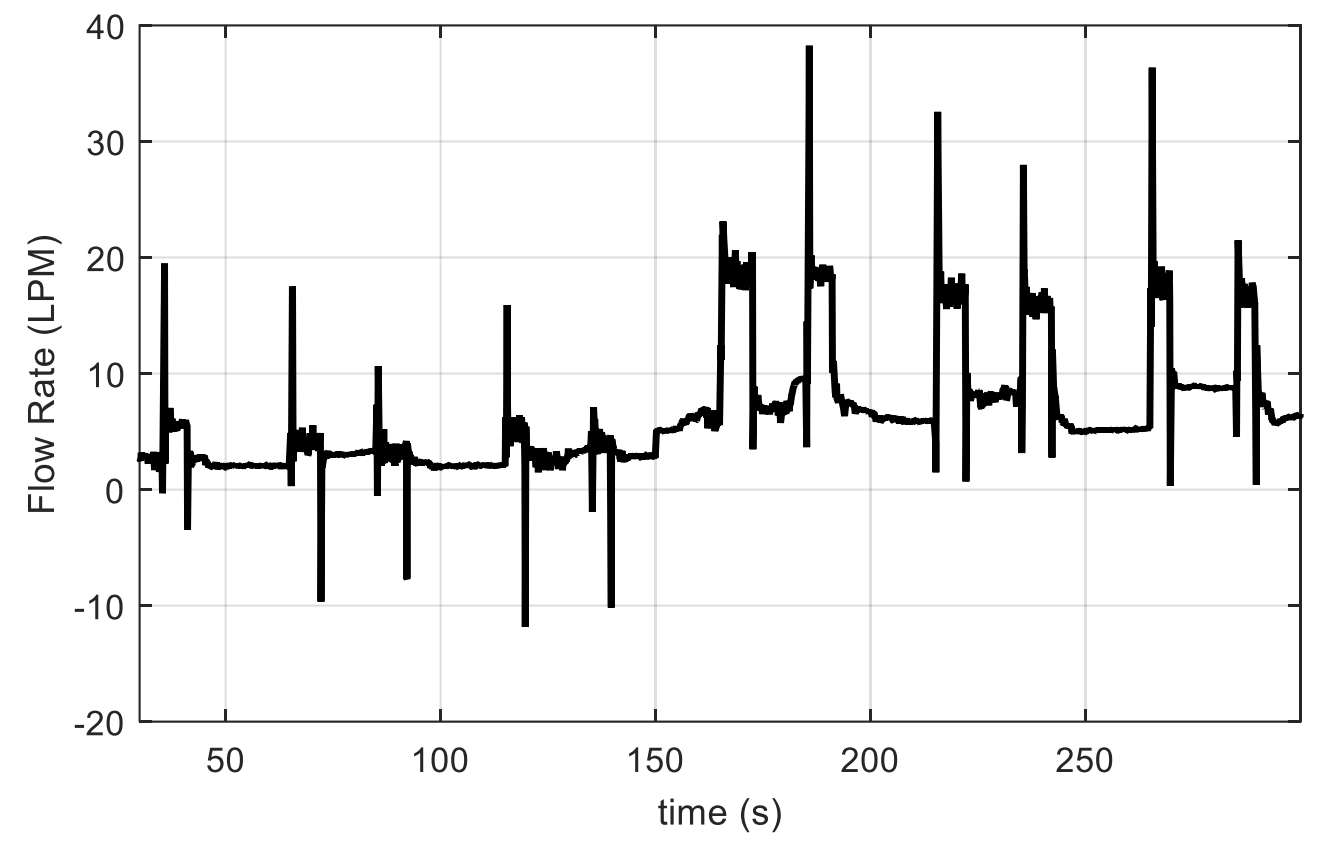

Figure 58. Flow loss estimation from the two state model utilizing the EKF with offset flow loss model.

All cases show a very similar result. The discharge pressure measurement compares extremely well to the estimated state. Furthermore, for all filter parameter estimations, at 150 seconds when the flow loss valve is opened up, the flow loss coefficient estimation is increased resulting in a higher flow loss flow estimation. Figure 59 compares the $K_{2}$ estimation from the first order model to the second order model. 


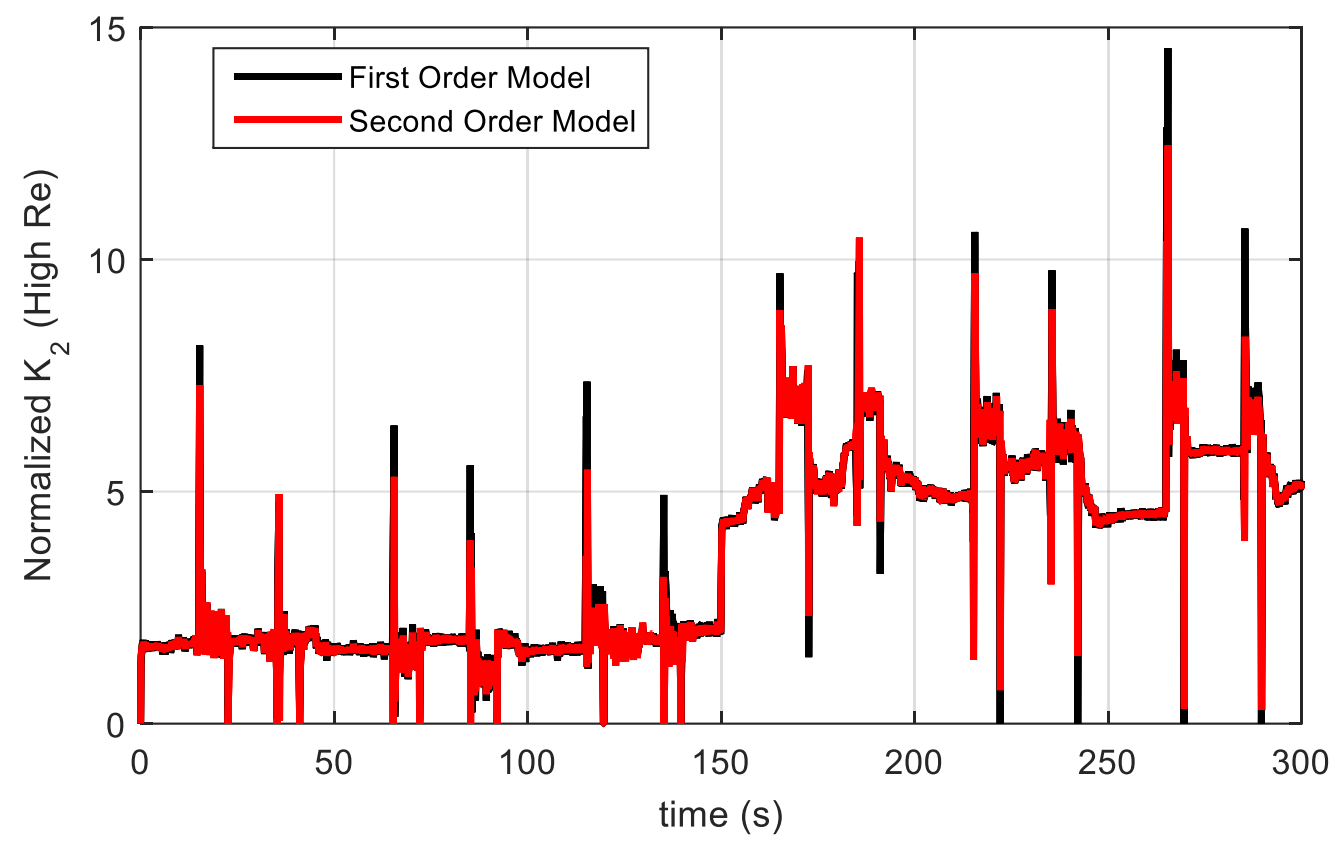

Figure 59. Comparison of K2 estimation from the first order model to the second order model. Modeling the swash plate does not have an apparent improvement over simply using the swash plate angle as an input only. The next section discussions situations when modeling the swash plate could be beneficial.

\subsection{Flow Loss Estimation without Swash Plate Angle Measurement}

Modeling the swash plate angle can still be useful, however. One of the drawbacks of these results is that the swash plate angle must be measured regardless of whether the swash plate angle is an input or a state. The feasibility of being able to measure the swash plate angle outside of a lab setting is questionable. Augmenting a pump to include a swash plate angle is far more complicated than say a pressure measurement. If swash plate angle could be determined from a different measurement, then modeling the swash plate angle would still be a valid choice. Consider Eq. (24). The control pressure is a function of both 
the discharge pressure and the swash plate angle, and could be used as a second measurement to determine the swash plate angle without measuring its position. A component was installed on the pump to be able to measure the control pressure. This modification is shown in Fig. 60.

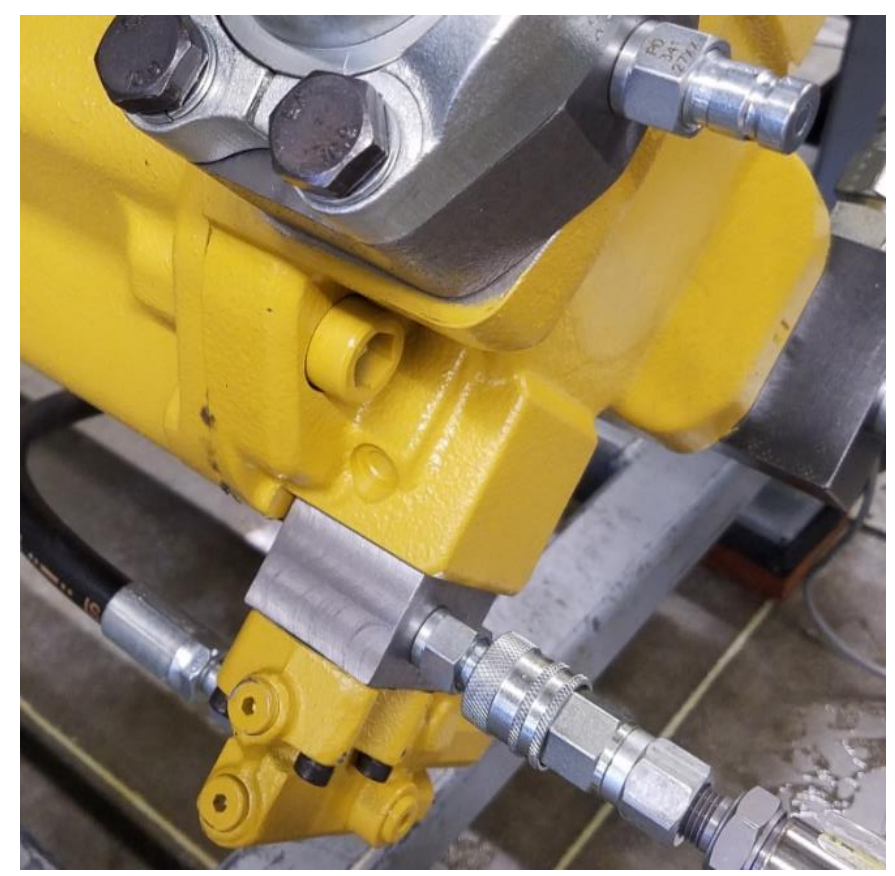

Figure 60. Additional Component for measuring control pressure.

The control pressure does have its own drawbacks. Figure 61 shows the control pressure from one of the experimental tests and FFT of the control pressure from 38 seconds to 40 seconds. The control pressure has fast oscillations. This high frequency oscillations presents a sampling problem for measuring the signal. Without a high sampling rate on the order of $1 \mathrm{kHz}$ or more, aliasing could become a serious problem. 
(a)

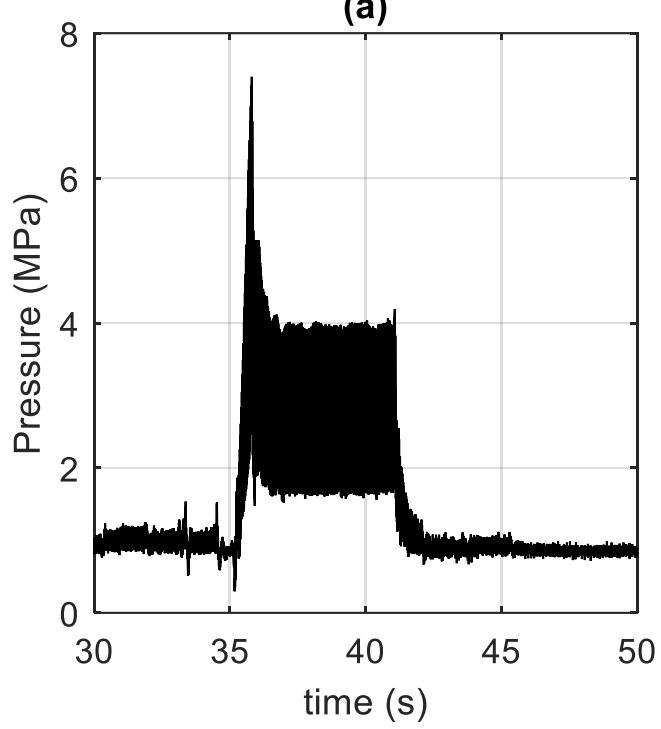

(b)

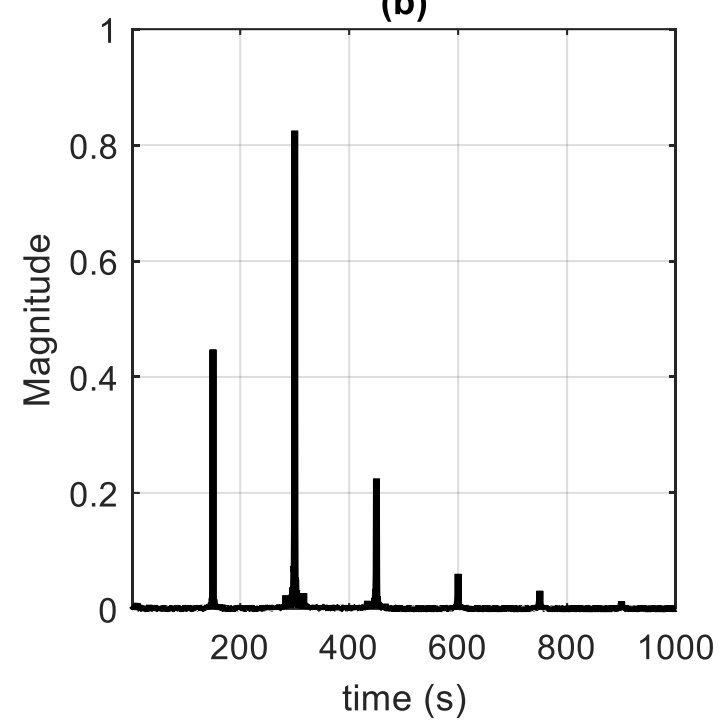

Figure 61. (a) Control pressure measurement during turning and (b) FFT of measurement from 38 seconds to 40 seconds.

One solution is to filter the control pressure. The high frequency oscillation of the control pressure does not show up in the swash plate angle measurement. Therefore, there must be some inertial dampening to cause the swash plate angle to not oscillate like the control pressure. Filtering the control pressure can be analogous to the inertial dampening. Figure 62 shows the result of applying a moving average window filter. The window size is 0.1 seconds. Note that in these experimental tests, sampling was done at $5 \mathrm{kHz}$, so applying the filter after the data collection is appropriate. A hardware filter may be necessary for a lower sampling rate. 


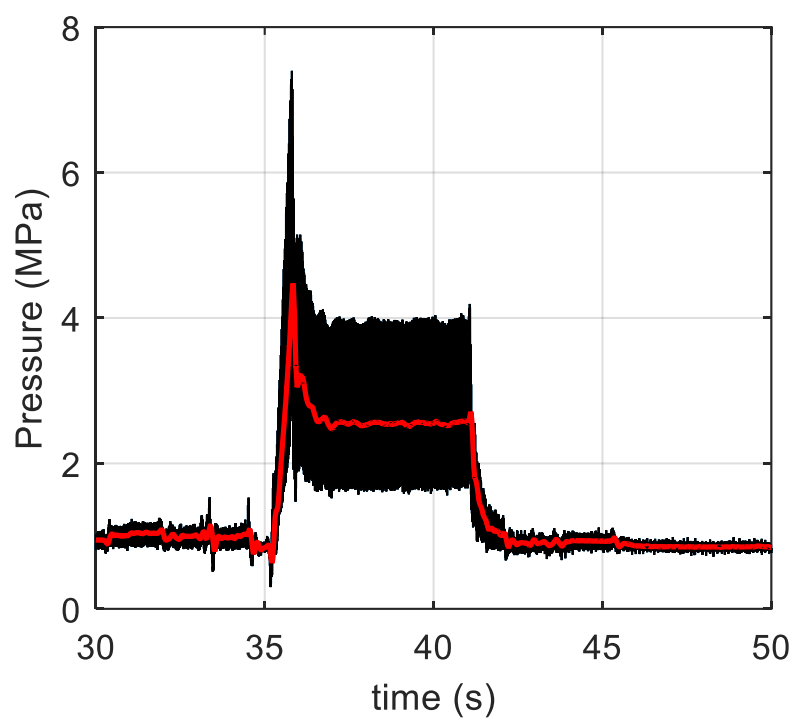

Figure 62. Comparison of filtered control pressure to unfiltered.

The EKF was implemented with a sample rate of $100 \mathrm{~Hz}$ utilizing the second order model, using the discharge pressure and control pressure as measurements. $K_{2}$ was the parameter estimated. Note that in Eq. (24), the control pressure has an offset term. The measurement should only be a function of the states. The output equation utilized for the control pressure was

$$
y_{P_{C}}=\frac{1}{A_{c p} L_{2 a}}\left(\left(A_{b p} L_{2 a}-C_{p}\right) P_{D}+\left(C_{i}-k_{b p} L_{2 a}^{2}\right) \alpha\right) .
$$

The offset was subtracted from the control pressure being utilized in the EKF, shown as

$$
z_{P_{C}}=P_{C}-\frac{1}{A_{c p} L_{2 a}}\left(k_{b p} L_{2 a}^{2}\left(\alpha_{\max }+x_{0}\right)\right)
$$

The process and measurement noise was

$$
v(t)=\left[\begin{array}{c}
1.73 e^{5} \\
2.66 e^{-3} \\
2.74 e^{-3}
\end{array}\right] \text { and } n(t)=\left[\begin{array}{l}
1.73 e^{5} \\
1.73 e^{5}
\end{array}\right]
$$




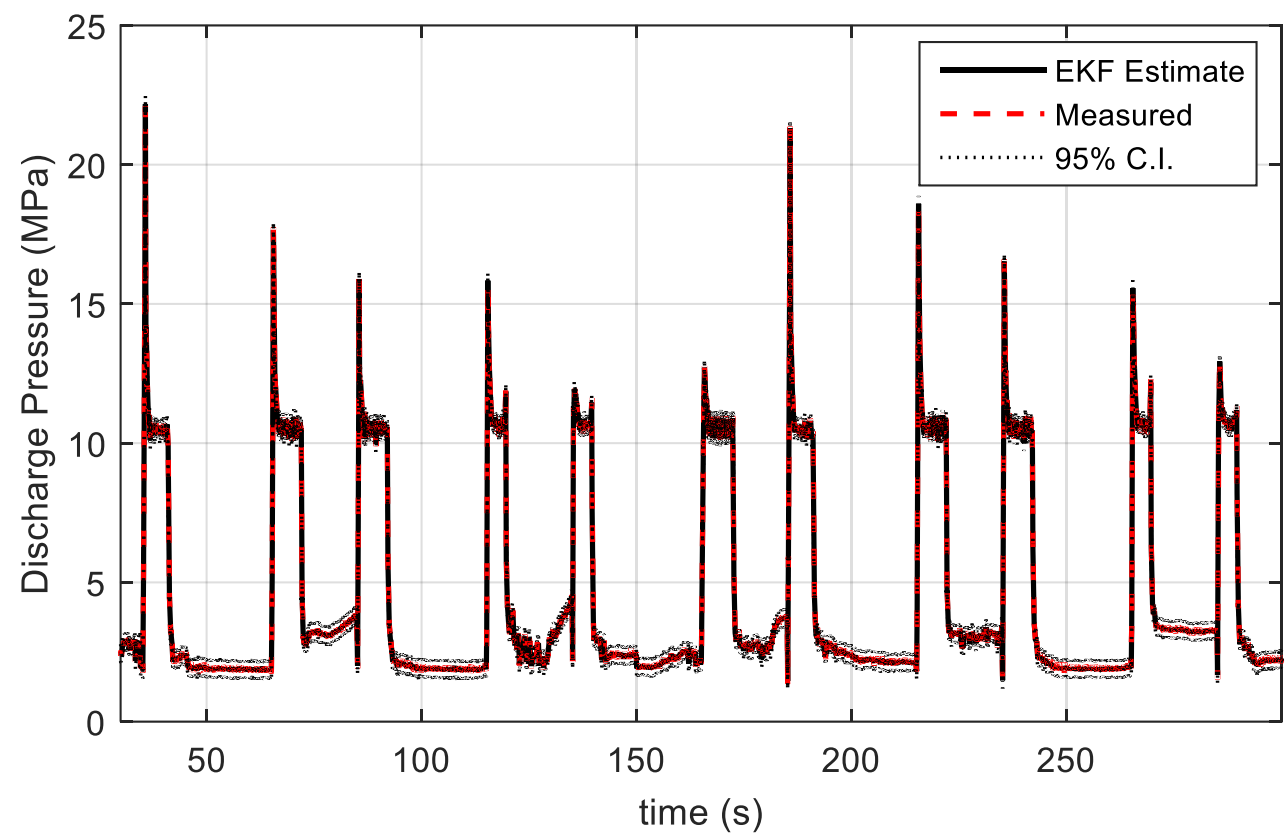

Figure 63. Discharge pressure estimation from the two state model utilizing the EKF with control pressure measured.

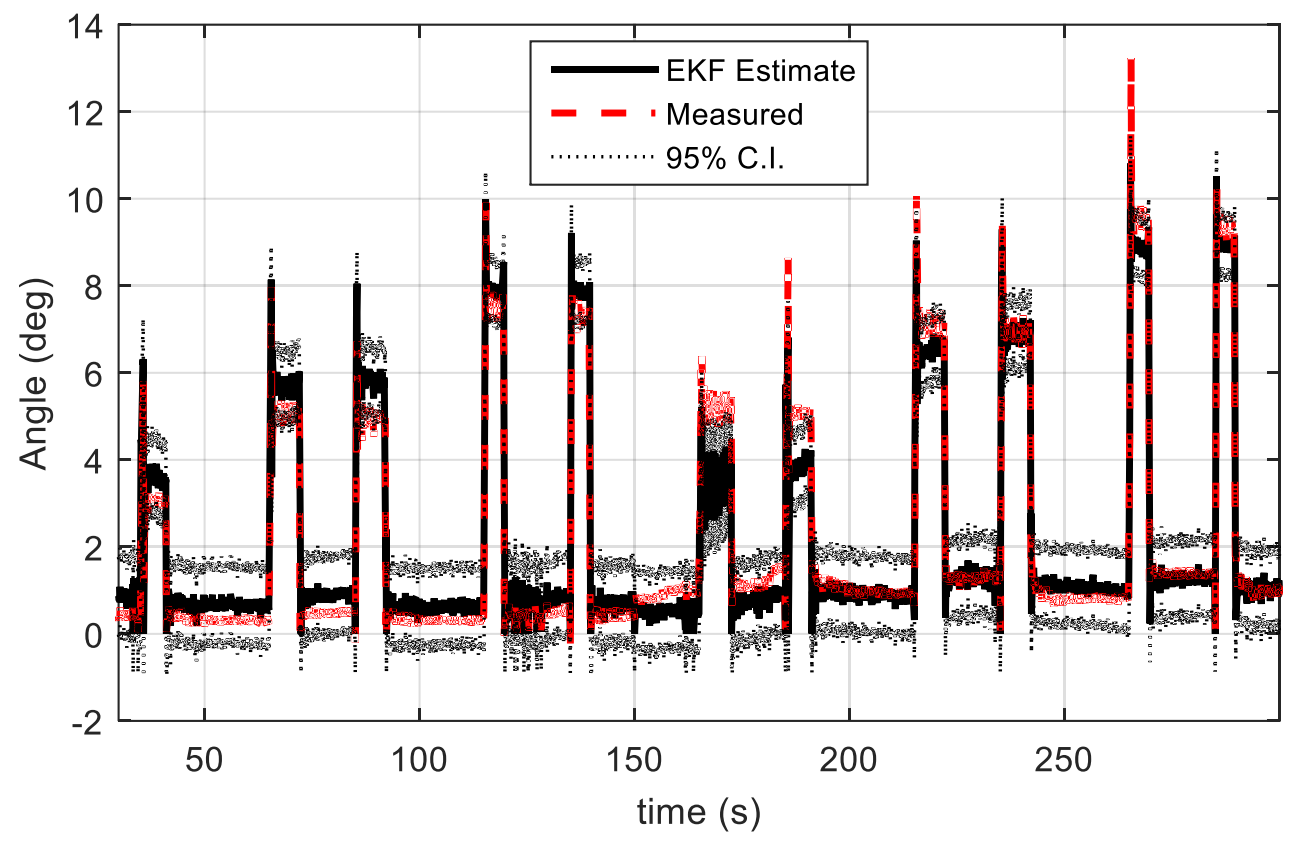

Figure 64. Swash plate angle estimation from the two state model utilizing the EKF with control pressure measured. 


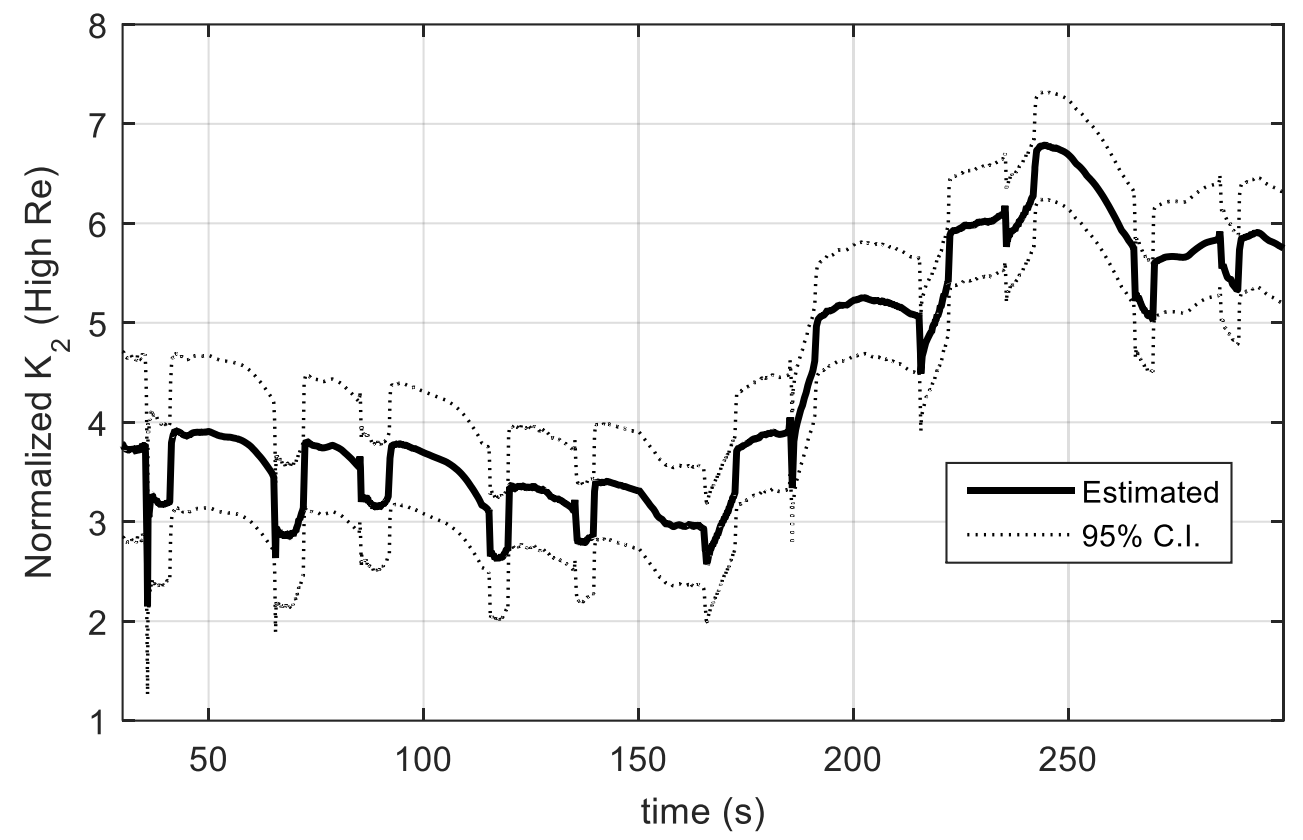

Figure 65. Flow loss coefficient estimation from the two state model utilizing the EKF with control pressure measured.

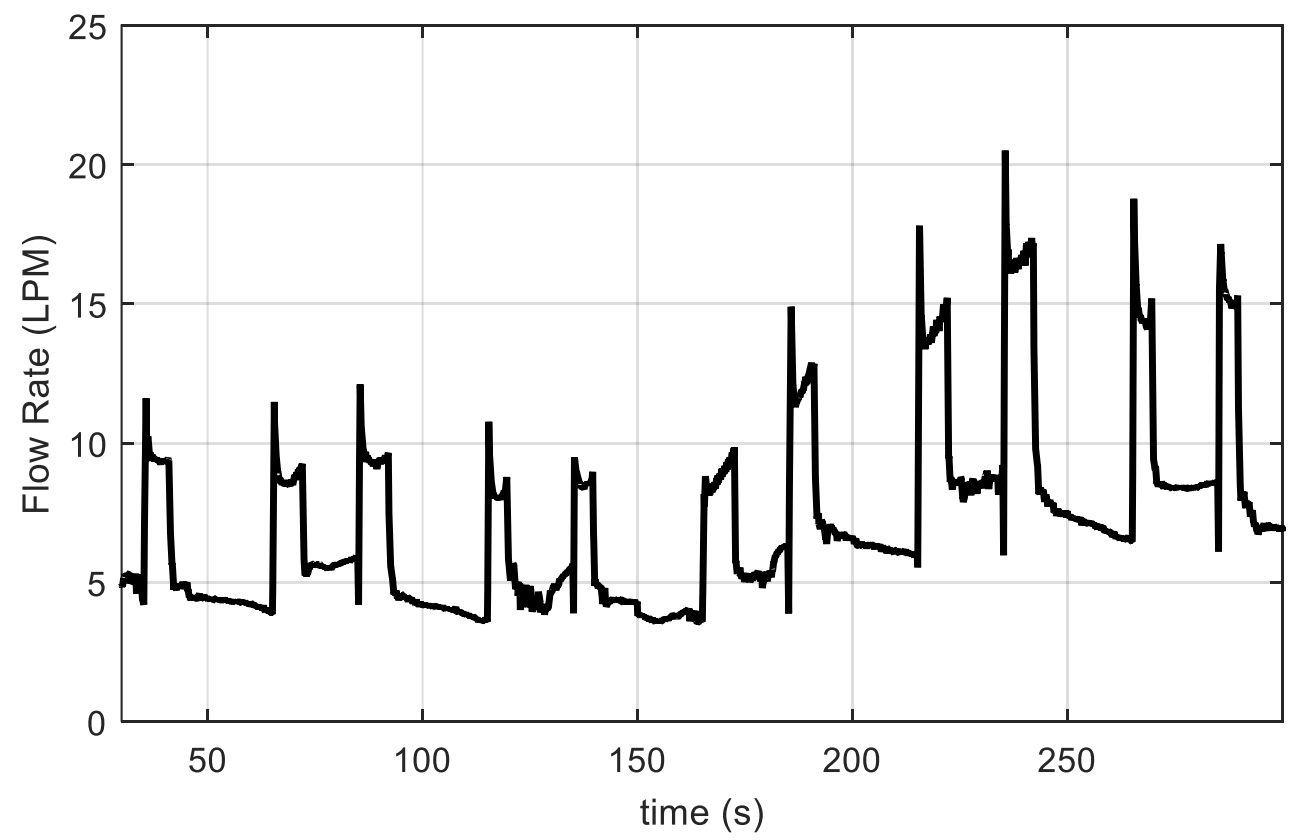

Figure 66. Flow loss estimation from the two state model utilizing the EKF with control pressure measured. 
Figure 67 compares the results of all the second order model flow loss estimations utilizing the EKF. Note that the single order model estimation with the EKF and UKF second order estimation is left off since the results were nearly identical to the second order model utilizing the EKF. The results from the general least squares has been included as well.

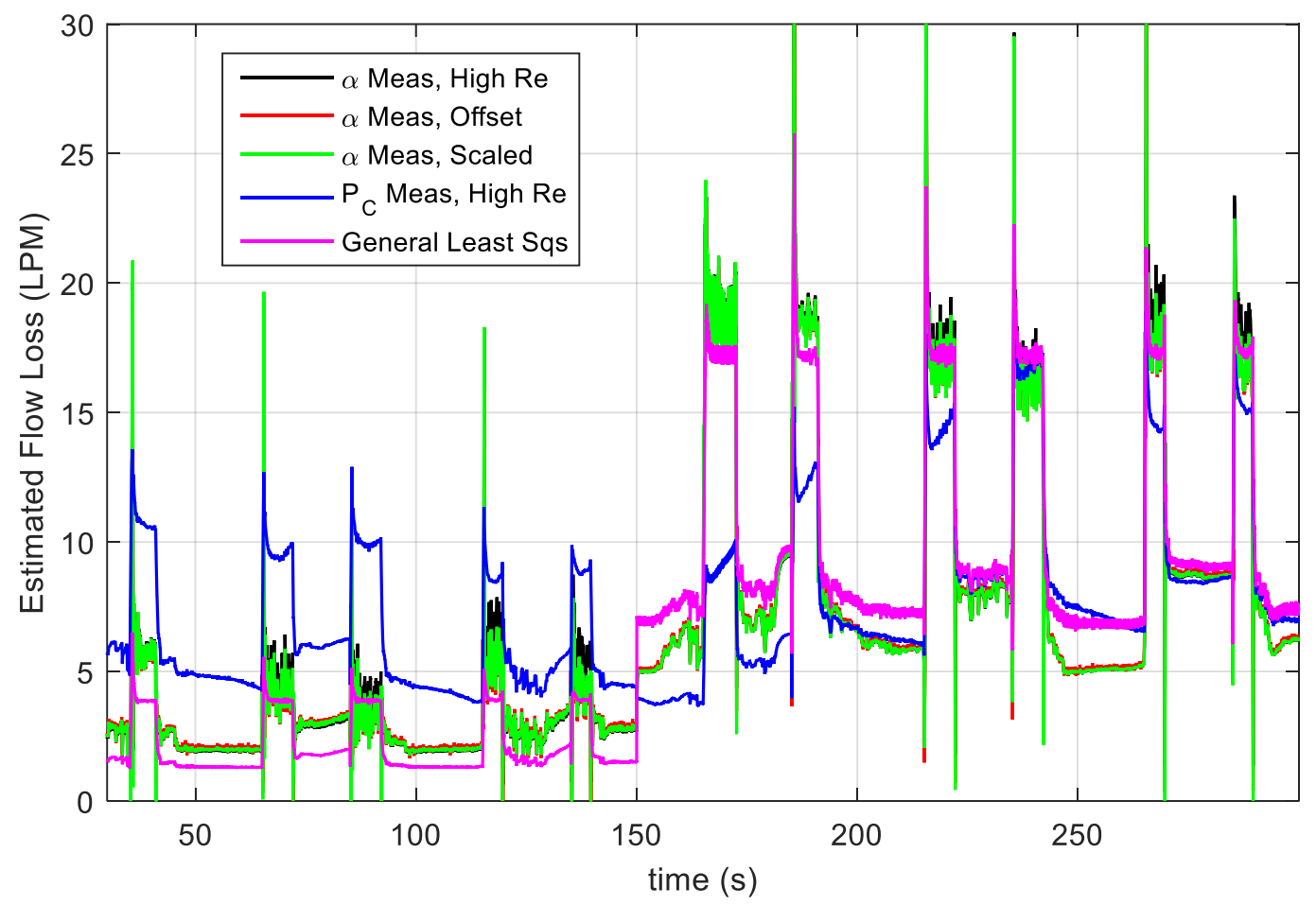

Figure 67. Comparison of flow loss estimation for four different estimations.

All estimation compares well, with the exception of using the control pressure to estimate the swash plate position. This result suggests that if the swash plate is measurable, the flow loss estimation can be accomplished by estimating $K_{2}, K_{3}$, or $K_{4}$. The results do not differ enough between these three estimation cases to suggest one is definitively better than the other two. Utilizing the control pressure measurement is clearly a worse estimation technique however. Furthermore, the complications associated with the control pressure 
measurement make this measurement unlikely to be utilized outside of a laboratory setting due to the need to modify the pump to place the control pressure sensor.

Table 4. Comparison of filter results.

\begin{tabular}{ccccc}
\hline \hline Model & Filter Technique & $\begin{array}{c}\text { Parameter } \\
\text { Estimated }\end{array}$ & $\begin{array}{c}\text { Flow Error } \\
(\text { LPM })\end{array}$ & $\begin{array}{c}\text { Flow Error During } \\
\text { Turning (LPM) }\end{array}$ \\
\hline 1 & EKF & $K_{2}$ & 1.32 & 2.08 \\
2 & UKF & $K_{2}$ & 1.21 & 1.81 \\
2 & EKF & $K_{2}$ & 1.20 & 1.69 \\
2 & EKF & $K_{3}$ & 1.16 & 1.44 \\
2 & EKF & $K_{4}$ & 1.17 & 1.49 \\
2 & EKF- using $P_{C}$ & $K_{2}$ & 3.14 & 4.82 \\
\hline
\end{tabular}

Table 4 compares the results of the shown filter estimations above. The filter estimation is compared to the general least squares fit to calculate an error. Recall that the general least squares results all have an $r^{2}$ value greater than 0.96 , which indicates the fit was accurate. All of the estimation techniques have a similar error, except for the estimation utilizing the control pressure signal which has a noticeably larger error. Note that the rest of the estimation utilized the swash plate angle sensor as either a measurement or input to the model. From this table, it is shown that the gain estimation and offset estimation are the best, although the high Reynolds number estimation is close. There is a noticeable decrease in estimation error between the first and second order models that was not apparent from the previous figures. The general least squares fit was only done during the turning phases, so the error was calculated during the turning phases only as well. The error does go up, but consider that the flow rate is much higher during turning.

The previous results show that flow loss changes can be estimated, but either the swash plate angle or the control pressure must be measured. As mentioned previously, the feasibility of doing utilizing these measurements outside a test condition is questionable. 
Swash plate angle can be known without measuring its position when the swash plate angle is at max displacement since the margin pressure, or pressure drop across the steering wheel valve, drops off significantly. An experimental test was done at a low RPM (450) to keep the swash plate angle at maximum displacement easily. The steering wheel was manually turned several times from endpoint to endpoint, then the leakage valve was opened, and the steering wheel was manually turned again. 
(a)

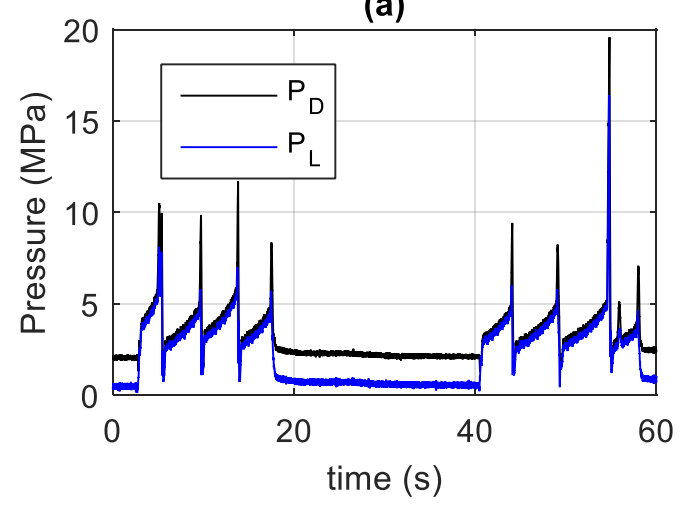

(c)

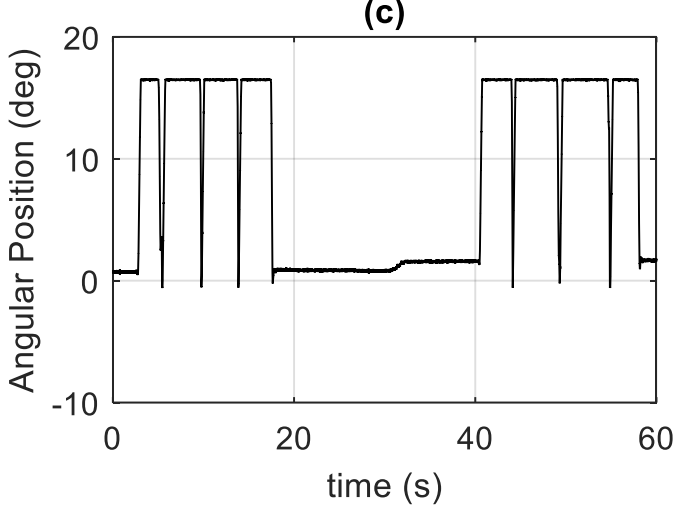

(e)

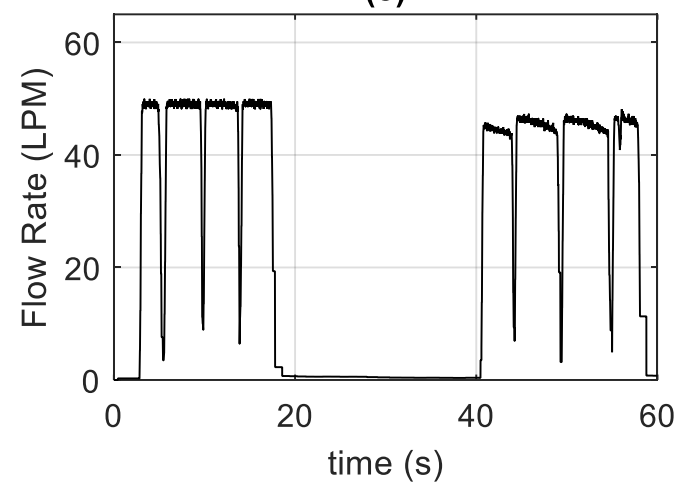

(b)

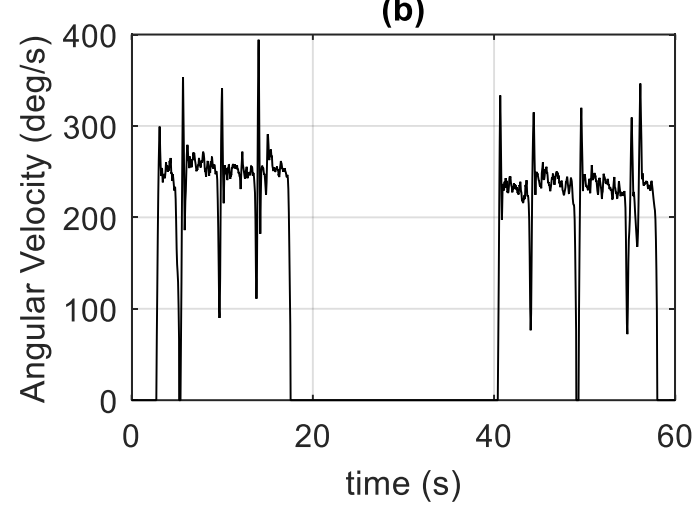

(d)

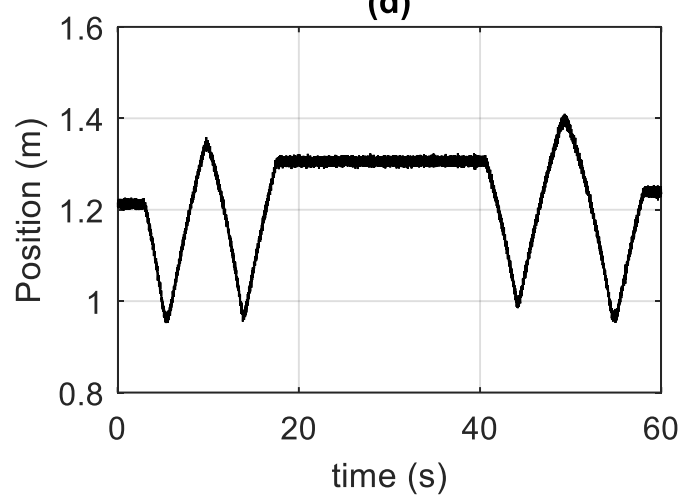

(f)

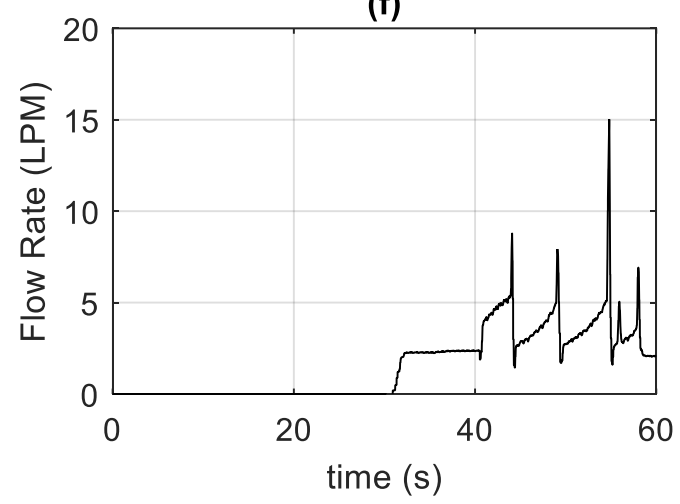

Figure 68. Experimental test for (a) discharge pressure (b) steering velocity (c) swash plate angle (d) cylinder displacement (e) discharge flow rate (f) leakage valve flow rate.

Recall from the modeling section that the flow rate across the steering wheel valve was inaccurate when the swash plate angle hits max displacement. The left cylinder position measurement was used instead. Note that the position was filtered utilizing a 0.15 
second moving window average, then differentiated, and filtered again. One way to filter would possibly to apply the EKF to the linkage model and using the estimated cylinder position but has not been done for this work. Figure 69 compares the measured discharge flow to the two estimated versions. Clearly, the estimation from the cylinder position measurement is far better.

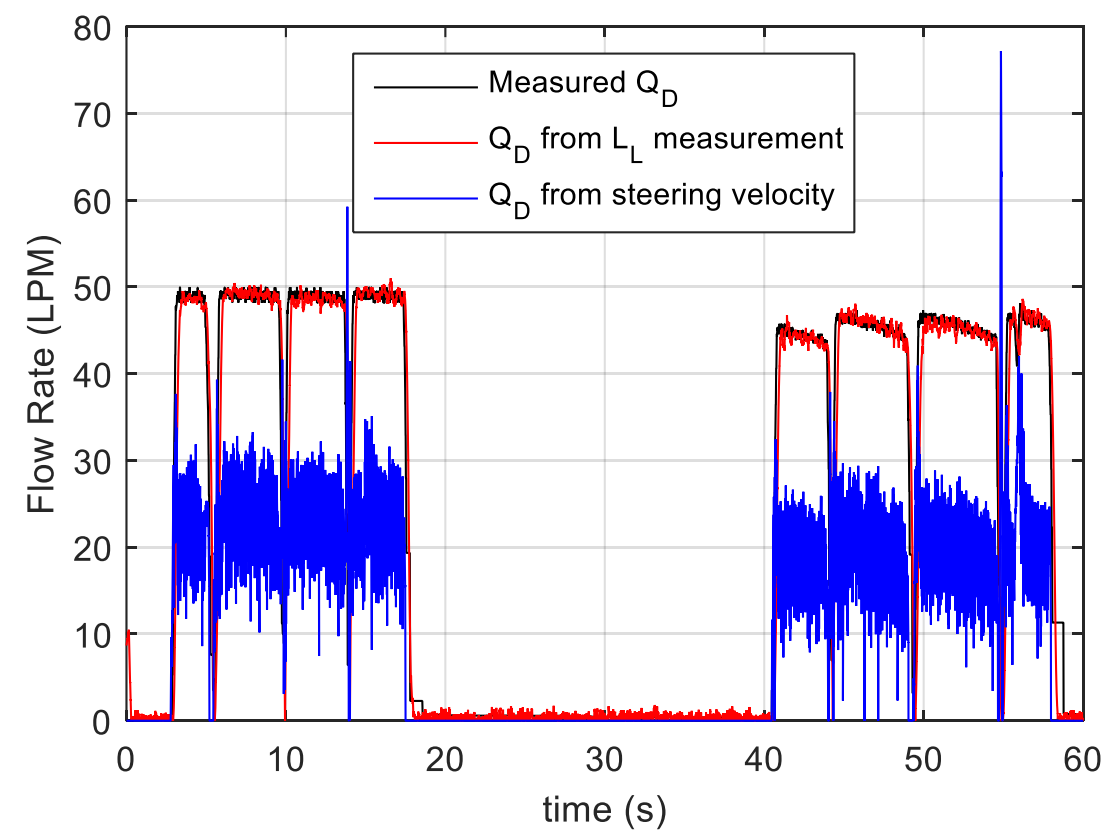

Figure 69. Comparison of measured flow to estimated flow across the steering wheel valve. Since swash plate angle is at maximum displacement, there is no reason to include the swash plate angle dynamic equation in the model. The first order model is used for the EKF algorithm, which is ran at $100 \mathrm{~Hz}$. The EKF only estimates when the margin pressure is below $1 \mathrm{MPa}$. Otherwise, the estimated discharge pressure is assumed to be the measured discharge pressure, and the flow loss coefficient is held constant. The results of the filter are shown in Figs. 70 and 71. 


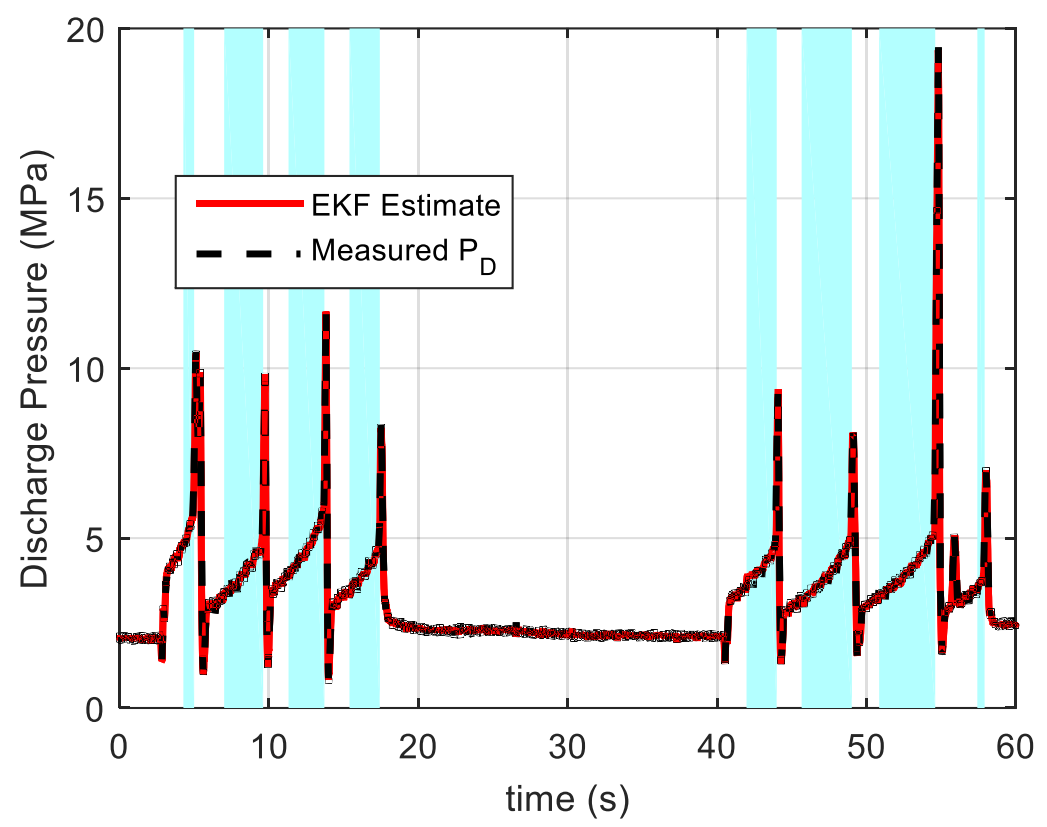

Figure 70. Discharge pressure estimation when swash plate angle is at maximum displacement (shaded portion indicates the maximum swashplate angle condition).

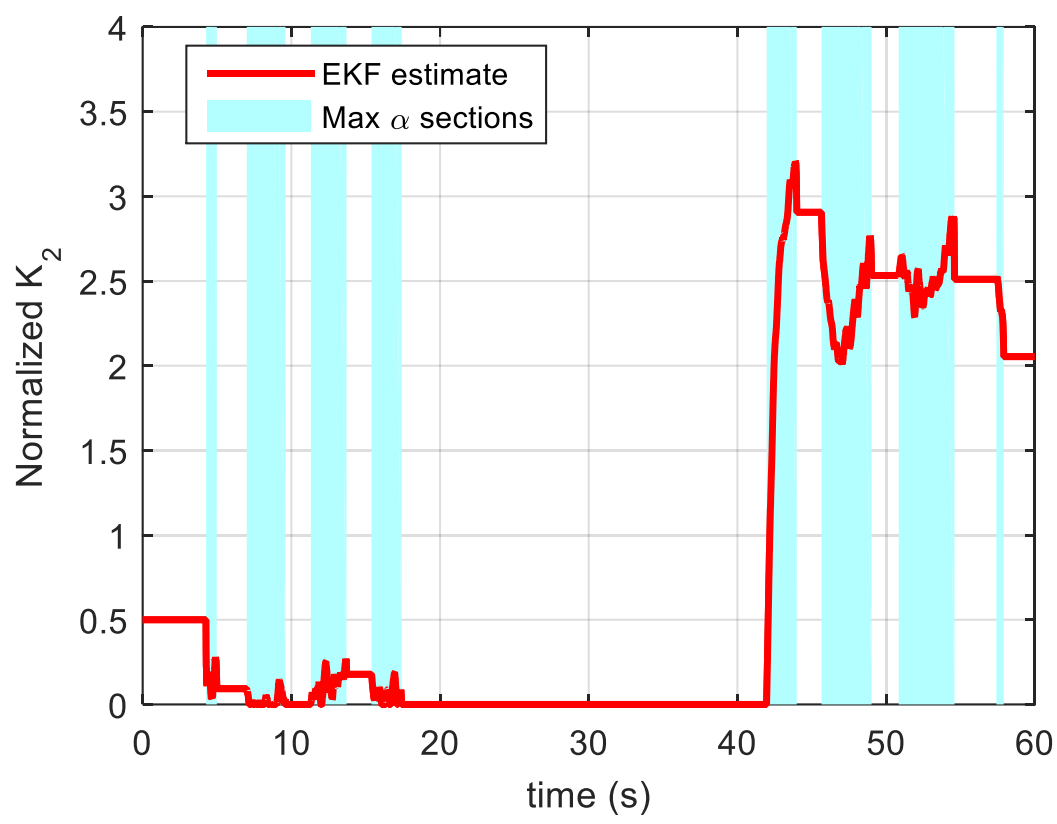

Figure 71. Flow loss coefficient estimation when swash plate angle is at maximum displacement (shaded portion indicates the maximum swashplate angle condition). 
The change in flow loss was detected. At 30 seconds, the loss valve was opened. When the margin pressure dipped below $1 \mathrm{MPa}$, indicated on the plots as the light blue sections, the flow loss estimation increased substantially compared to the estimation when the valve is closed. 


\section{CHAPTER 7: CONCLUSION}

\subsection{Summary of Findings}

Several stochastic filtering techniques were considered for estimating and tracking changing flow loss parameters of an axial piston pump. The Kalman filter, pole placement filter, extended Kalman filter, unscented Kalman filter, and particle filter were all considered. Particularly the EKF was proposed for implementing flow loss estimation. The combination of superior runtime to the UKF and PF and better estimation than the KF and PPF make the EKF an adequate estimation technique in real time.

The system was found to be observable with the discharge pressure as a filter measurement, and swash plate angle, load pressure, and steering wheel velocity (or left cylinder position) as inputs to the model. The control pressure measurement could also be utilized instead of the swash plate angle measurement without loss of observability, though with some decrease in estimation accuracy. Furthermore, no measurement was found to be needed for the swash plate angle if the pump was operated at a max flow, which was detectable given the margin pressure measurement.

Results were compared to a general least squares fit of the flow loss parameters. The EKF estimation improves upon this estimation by allowing for real time estimation of the flow loss parameter. Furthermore, the EKF is an improvement over the general least squares fit because of the EKF's ability to estimate a flow loss parameter for a single steady state operating point.

Despite the high complexity of the hydraulic system, this work demonstrates that a simpler approach is sufficient for flow loss estimation. A single pressure state is sufficient 
for determining flow losses of an axial piston pump. This simplification has several advantages. The first and second order models presented here can be ran at a sample rate comparable to industry measurement rates, making the models ideal for filter usage. The two models are also present an simpler linearization compared to high order nonlinear hydraulic models, which often have complicated linearizations due to numerous discontinuous functions present in the model.

\subsection{Areas for Future Research}

There is great potential for future research projects relating to this work. Accurate flow loss estimation without swash plate angle and control pressure measurement would be a novel breakthrough. The laboratory setup utilized for this work had access to both these sensors, but current industry setups do not often have these two sensors. This work demonstrated that estimation without those sensors is possible when the margin pressure decreases, and the swash plate angle is assumed to be at its maximum displacement. Estimation during other periods is the next step for this work. Estimation without swash plate angle or control pressure measurement is a difficult problem to solve. Current analysis of the pump suggests that flow loss has little effect on the pressure dynamics. Therefore, determining the flow loss from those pressure measurements is difficult. Further research could explore how to accurately estimate swash plate angle without swash plate angle measurement.

The eighth order model was found to be observable without either measurement of swash plate angle or control pressure. Given the discharge pressure and load pressure, the swash plate angle could be known from an accurate three-way control valve and control 
pressure dynamic model. Modeling these is what led to the eighth order model being impractical in terms of simulation step size, however. Further research could focus on modeling the control valve and control pressure dynamics in a way that allows for larger step time simulations. The control pressure and control valve position have sustained, high frequency oscillations under normal operation as observed in experiments and simulations. However, modeling these states without oscillation to where their steady state values converge to the mean value of the oscillations may work, although this has not been explored in the work presented here. 


\section{REFERENCES}

[1] Z. Peng and F. Chu, "Application of the wavelet transform in machine condition monitoring and fault diagnostics: a review with bibliography," Mechanical systems and signal processing, vol. 18, no. 2, pp. 199-221, 2004.

[2] F. Kong and R. Chen, "A combined method for triplex pump fault diagnosis based on wavelet transform, fuzzy logic and neuro-networks," Mechanical Systems and Signal Processing, vol. 18, no. 1, pp. 161-168, 2004.

[3] V. Muralidharan and V. Sugumaran, "Feature extraction using wavelets and classification through decision tree algorithm for fault diagnosis of mono-block centrifugal pump," Measurement, vol. 46, no. 1, pp. 353-359, 2013.

[4] W. T. Peter, Y. Peng, and R. Yam, "Wavelet analysis and envelope detection for rolling element bearing fault diagnosis - their effectiveness and flexibilities," Journal of vibration and acoustics, vol. 123, no. 3, pp. 303-310, 2001.

[5] K. M. Hancock and Q. Zhang, "A hybrid approach to hydraulic vane pump condition monitoring and fault detection," Transactions of the ASABE, vol. 49, no. 4, pp. 1203-1211, 2006.

[6] L. An and N. Sepehri, "Leakage fault identification in a hydraulic positioning system using extended Kalman filter," in American Control Conference, 2004. Proceedings of the 2004, 2004, vol. 4, pp. 3088-3093: IEEE.

[7] L. An and N. Sepehri, "Hydraulic actuator leakage fault detection using extended Kalman filter," International Journal of Fluid Power, vol. 6, no. 1, pp. 41-51, 2005.

[8] X. Cui, Y. Dong, and K. Zhao, "Measurement of external leakage of hydraulic servo-motor based on robust extended kalman filter," in Electronic Measurement \& Instruments, 2009. ICEMI'09. 9th International Conference on, 2009, pp. 2-6802-684: IEEE.

[9] J. Hindman, R. Burton, and G. Schoenau, "Condition monitoring of fluid power systems: A survey," SAE Technical Paper0148-7191, 2002.

[10] Y. Chinniah, R. Burton, and S. Habibi, "Viscous damping coefficient and effective bulk modulus estimation in a high performance hydrostatic actuation system using extended Kalman filter," International Journal of Fluid Power, vol. 4, no. 3, pp. 27-34, 2003.

[11] Y. Chinniah, R. Burton, and S. Habibi, "Failure monitoring in a high performance hydrostatic actuation system using the extended Kalman filter," Mechatronics, vol. 16, no. 10, pp. 643-653, 2006.

[12] L. Ljung, "Asymptotic behavior of the extended Kalman filter as a parameter estimator for linear systems," IEEE Transactions on Automatic Control, vol. 24, no. 1, pp. 36-50, 1979.

[13] J. A. Bryan and R. C. Fales, "Estimating Cutting Force Coefficients in High-Speed Low-Radial-Immersion Milling Processes," in ASME 2014 Dynamic Systems and Control Conference, 2014, pp. V002T35A002-V002T35A002: American Society of Mechanical Engineers.

[14] M. N. Said, M. E. H. Benbouzid, and A. Benchaib, "Detection of broken bars in induction motors using an extended Kalman filter for rotor resistance sensorless estimation," IEEE Transactions on energy conversion, vol. 15, no. 1, pp. 66-70, 2000 . 
[15] G. L. Plett, "Extended Kalman filtering for battery management systems of LiPBbased HEV battery packs: Part 3. State and parameter estimation," Journal of Power sources, vol. 134, no. 2, pp. 277-292, 2004.

[16] P. L. Houtekamer and H. L. Mitchell, "Ensemble kalman filtering," Quarterly Journal of the Royal Meteorological Society, vol. 131, no. 613, pp. 3269-3289, 2005.

[17] G. Evensen, "The ensemble Kalman filter: Theoretical formulation and practical implementation," Ocean dynamics, vol. 53, no. 4, pp. 343-367, 2003.

[18] H. Moradkhani, S. Sorooshian, H. V. Gupta, and P. R. Houser, "Dual stateparameter estimation of hydrological models using ensemble Kalman filter," Advances in water resources, vol. 28, no. 2, pp. 135-147, 2005.

[19] A. Schöniger, W. Nowak, and H. J. Hendricks Franssen, "Parameter estimation by ensemble Kalman filters with transformed data: Approach and application to hydraulic tomography," Water Resources Research, vol. 48, no. 4, 2012.

[20] N. J. Gordon, D. J. Salmond, and A. F. Smith, "Novel approach to nonlinear/nonGaussian Bayesian state estimation," in IEE Proceedings F (Radar and Signal Processing), 1993, vol. 140, no. 2, pp. 107-113: IET.

[21] E. A. Wan and R. Van Der Merwe, "The unscented Kalman filter for nonlinear estimation," in Adaptive Systems for Signal Processing, Communications, and Control Symposium 2000. AS-SPCC. The IEEE 2000, 2000, pp. 153-158: Ieee.

[22] T. Shinn, R. Carpenter, and R. C. Fales, "State Estimation Techniques for Axial Piston Pump Health Monitoring," in ASME/BATH 2015 Symposium on Fluid Power and Motion Control, 2015, pp. V001T01A065-V001T01A065: American Society of Mechanical Engineers.

[23] T. Shinn, R. Carpenter, and R. C. Fales, "Pump Efficiency Parameter Estimation," in BATH/ASME 2016 Symposium on Fluid Power and Motion Control, 2016, pp. V001T01A030-V001T01A030: American Society of Mechanical Engineers.

[24] N. D. Manring, "Mapping the efficiency for a hydrostatic transmission," Journal of Dynamic Systems, Measurement, and Control, vol. 138, no. 3, p. 031004, 2016.

[25] N. D. Manring, Fluid power pumps and motors: analysis, design and control. McGraw Hill Professional, 2013.

[26] Z. D. Wagner and R. Fales, "Modeling and stability of a hydraulic load-sensing pump with investigation of a hard nonlinearity in the pump displacement control system," in ASME/BATH 2014 Symposium on Fluid Power and Motion Control, 2014, pp. V001T01A001-V001T01A001: American Society of Mechanical Engineers.

[27] S. S. Haykin, Kalman filtering and neural networks. Wiley Online Library, 2001.

[28] K. G. Jöreskog and D. Sörbom, LISREL VI: Analysis of linear structural relationships by maximum likelihood, instrumental variables, and least squares methods. Scientific Software, 1986.

[29] R. G. Brown and P. Y. Hwang, "Introduction to random signals and applied Kalman filtering: with MATLAB exercises and solutions," Introduction to random signals and applied Kalman filtering: with MATLAB exercises and solutions, by Brown, Robert Grover.; Hwang, Patrick YC New York: Wiley, c1997., 1997. 
[30] M. S. Arulampalam, S. Maskell, N. Gordon, and T. Clapp, "A tutorial on particle filters for online nonlinear/non-Gaussian Bayesian tracking," IEEE Transactions on signal processing, vol. 50, no. 2, pp. 174-188, 2002.

[31] S. J. Julier, "The scaled unscented transformation," in American Control Conference, 2002. Proceedings of the 2002, 2002, vol. 6, pp. 4555-4559: IEEE. 


\section{VITA}

Tyler Shinn grew up in Quincy, Illinios and graduated from Quincy Senior High school in June 2009. In May 2013, he graduated with a BS in Mechanical and Aerospace Engineering from University of Missouri. He continued his schooling by getting a MS in Mechanical and Aerospace Engineering from the University of Missouri in August 2015. He is finishing his Ph.D. in Mechanical and Aerospace Engineering in May of 2018. The focus of his research has been in dynamic modeling, automatic control, hydraulic systems, and estimation techniques. 